\title{
Automated Pre-Processing and Automated Post-Processing in EEG/MEG Brain Source ANALYSIS
}

\author{
by \\ Seyed-Youns Sadat-Nejad \\ Bachelor of Biomedical Engineering, Ryerson, 2017
}

A thesis
presented to Ryerson University

in partial fulfillment of the

requirements for the degree of

Master of Applied Science

in the Program of

Electrical and Computer Engineering

Toronto, Ontario, Canada, 2019

(C)Seyed-Youns Sadat-Nejad 2019 


\section{AUTHOR'S DECLARATION FOR ELECTRONIC SUBMISSION OF A THESIS}

I hereby declare that I am the sole author of this thesis. This is a true copy of the thesis, including any required final revisions, as accepted by my examiners.

I authorize Ryerson University to lend this thesis to other institutions or individuals for the purpose of scholarly research.

I further authorize Ryerson University to reproduce this thesis by photocopying or by other means, in total or in part, at the request of other institutions or individuals for the purpose of scholarly research.

I understand that my dissertation may be made electronically available to the public. 
Automated Pre-Processing and Automated Post-Processing in EEG/MEG Brain Source Analysis

Master of Applied Science 2019

Seyed-Youns Sadat-Nejad

Electrical and Computer Engineering

Ryerson University

\begin{abstract}
Analyzing Electroencephalography (EEG)/Magnetoencephalography (MEG) brain source signals allows for a better understanding and diagnosis of various brain-related activities or injuries. Due to the high complexity of the mentioned measurements and their low spatial resolution, different techniques have been employed to enhance the quality of the obtained results. The objective of this work is to employ state-of-the-art approaches and develop algorithms with higher analysis reliability. As a pre-processing method, subspace denoising and artifact removal approaches are taken into consideration, to provide a method that automates and improves the estimation of the Number of Component (NoC) for artifacts such as Eye Blinking (EB). By using synthetic EEG-like simulation and real MEG data, it is shown that the proposed method is more reliable over the conventional manual method in estimating the NoC. For Independent Component Analysis (ICA)-based approaches, the proposed method in this thesis provides an estimation for the number of components with an accuracy of $98.7 \%$. The thesis is also devoted to improving source localization techniques, which aims to estimate the location of the source within the brain, which elicit time-series measurements. In this context, after obtaining a practical insight into the performance of the popular L2-Regularization based approaches, a post-processing thresholding method is introduced. The proposed method improves the spatial resolution of the L2-Regularization inverse solutions, especially for Standard Low-Resolution Electromagnetic Tomography (sLORETA), which is a well-known and widely used inverse solution. As a part of the proposed method, a novel noise variance estimation is introduced, which combines the kurtosis statistical parameter and data (noise)
\end{abstract}


entropy. This new noise variance estimation technique allows for a superior performance of the proposed method compared to the existing ones. The algorithm is validated on the synthetic EEG data using well-established validation metrics. It is shown that the proposed solution improves the resolution of conventional methods in the process of thresholding/denoising automatically and without loss of any critical information. 


\section{Acknowledgements}

First of all, I would like to thank my supervisor, Dr.Soosan Beheshti, for her patient guidance, encouragement, kindness, and support. I am extremely grateful and lucky to have a supervisor that cared so much for my work and future. I want to thank her for allowing me to develop my autonomy by trusting me to go my way while ensuring that I am developing a researcher characteristic and gaining very in-depth mathematical and engineering knowledge.

Furthermore, I would like to thank the members of my thesis committee: Dr.Dimitri Androutsos, Dr.Karthi Umapathy, and Dr.Alagan Anpalagan for putting the time to read and evaluate my thesis and provide me with valuable feedback. I also want to thank them for their continuous support and help through my academic years.

I had a great experience at Ryerson University and many professors, students and staffs played a huge role in making it memorable and life-changing. I would like to thank this community for this great experience.

Last but not least, I would like to send my most profound appreciation and gratitude to my family for their love and supports throughout all my academic years. 


\section{Contents}

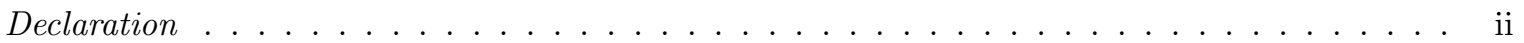

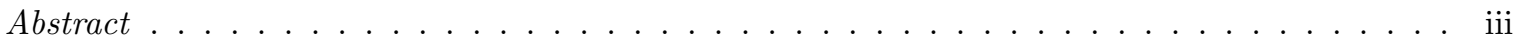

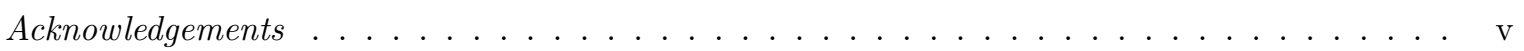

List of Tables . . . . . . . . . . . . . . . . . . . . viii

List of Figures . . . . . . . . . . . . . . . . . . . . ix

1 Introduction $\quad 1$

1.1 General View and Motivation . . . . . . . . . . . . . . . . . . . 1

1.2 Thesis Summary . . . . . . . . . . . . . . . . . . . 2

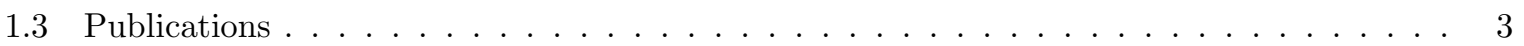

2 Background on Basic Concepts and General Assumptions 4

2.1 Introduction to Brain Anatomy and Structure of Neurons . . . . . . . . . . . . . . . 4

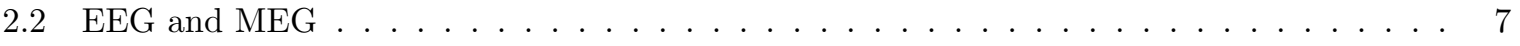

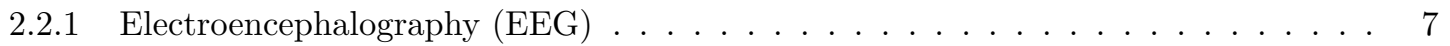

2.2 .2 Magnetoencephalography $(\mathrm{MEG}) \ldots \ldots \ldots \ldots \ldots \ldots \ldots$

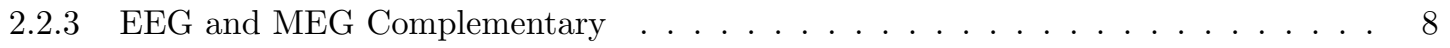

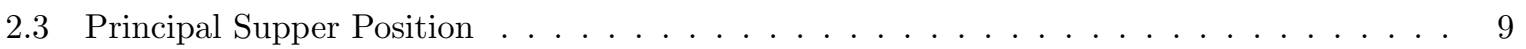

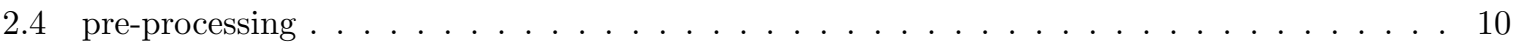

2.4.1 Time Sampling, Electrode Positioning and referencing . . . . . . . . . . . . 10

2.4 .2 Frequency Filtering . . . . . . . . . . . . . . . . . 12

2.4.3 Artifact Removal With Subspace Approaches . . . . . . . . . . . . . . . . . 12

2.4.4 Computationally Complex Pre-Processing Methods . . . . . . . . . . . . . . . . 16

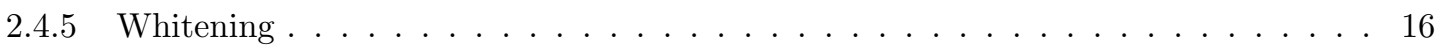

2.5 Overview of Forward Model and Inverse Model in Brain Source Localization . . . . . . . . 16

2.5.1 General Overview and Formulation in Brain Source Localization . . . . . . . . . . 17

2.6 Forward Model . . . . . . . . . . . . . . . . . . . . . 20

2.7 Inverse Model . . . . . . . . . . . . . . . . . . . . . . . . . . 22

2.7.1 Regularization-Based Methods . . . . . . . . . . . . . . . . 23

2.7 .2 Computationally Complex Inverse Solutions . . . . . . . . . . . . . . . 26

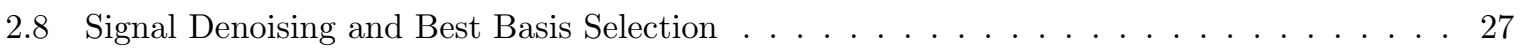


3 Reliable Automated pre-processing $\quad \mathbf{2 8}$

3.1 Ambiguities of Independent Component Analysis (ICA) and Signal Space Projection(SSP) 28

3.2 Ocular Artifact $(\mathrm{OA})$ Removal by Subspace Methods . . . . . . . . . . . . . . . . . . . 28

3.3 Eye Blink (EB) Number of Components (NoC) Estimate as Application of Number of Source Eigenvalue Error NoSEE _ . . . . . . . . . . . . . . . . . . 29

3.3.1 Step-By-Step Procedure of The Proposed NoSEE application . . . . . . . . . . . . 31

3.4 Simulation Results of the Proposed pre-processing Method . . . . . . . . . . . . . . . . 32

3.4.1 Synthetic EEG Data for the Proposed pre-processing Method . . . . . . . . . . . 32

3.4 .2 Real Magnetoencephalography (MEG) Data . . . . . . . . . . . . . . . . . 33

3.4.3 Evaluation Criteria of the Proposed pre-processing Method . . . . . . . . . . . 33

3.4.4 Synthetic Electroencephalography (EEG) Result and Discussion for the Proposed pre-processing Method . . . . . . . . . . . . . . . . . . . 34

3.4.5 Real MEG Result and Discussion of Proposed pre-processing Method . . . . . . . 36

4 Automated Source Thresholding in Low Resolution Inverse Problems 38

4.1 AESET in $L_{2}$-Regularization Inverse Problems $\ldots \ldots \ldots \ldots$

4.2 Automated EEG Source Error Thresholding (AESET) … . . . . . . . . . . . 39

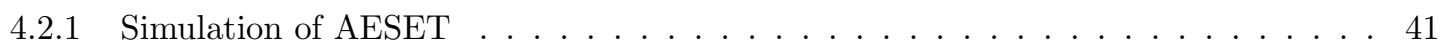

4.2 .2 Evaluation Criteria for AESET method . . . . . . . . . . . . . . . 42

4.2 .3 Simulation Results of AESET . . . . . . . . . . . . . . . . . . . . 42

4.2.4 Analysis of the Obtained Results for Further Improvements on AESET method . . 44

4.3 HR-sLORETA in EEG Source Imaging . . . . . . . . . . . . . . . . . . 45

4.3 .1 Noise Variance Estimation Using Kurtosis Analysis . . . . . . . . . . . . . . . . 45

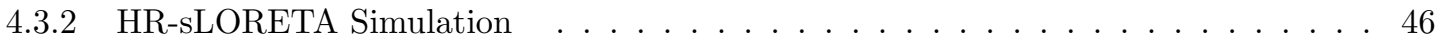

4.3 .3 Evaluation Criteria for HR-sLORETA . . . . . . . . . . . . . . . . 47

4.3.4 Simulation Result and Discussion of HR-sLORETA . . . . . . . . . . . . . 48



4.4.1 Noise Variance Estimation Using Kurtosis and Entropy Analysis . . . . . . . . . 50

4.4 .2 Improved HR-sLORETA Simulation and Evaluation . . . . . . . . . . . . . 52

4.4.3 Simulation Result and Discussion of Improved HR-sLORETA . . . . . . . . . . . 52

4.5 Additional Observation on The Effect of the Added Constraint in Regularization-Based models . . . . . . . . . . . . . . . . . . . . . . 54

5 Conclusion and Implications for Future Research $\quad 56$

$\begin{array}{ll}\text { Appendices } & 58\end{array}$

A Mathematical Simplification of The Error Reconstruction Algorithm $\quad 59$

$\begin{array}{ll}\text { References } & \mathbf{7 0}\end{array}$

$\begin{array}{ll}\text { Acronyms } & 71\end{array}$ 


\section{List of Tables}

3.1 Root Mean Square Error (RMSE) of Signal Space Projection (SSP), runICA and JADE artifact removals . $C(i)$ represents choosing the first $i$ components. Last column illustrates RMSE as well as the optimum chosen Number of Component (NoC) by the proposed method (in parenthesis). Last row is the averaged results over 75 trials. . . . . . . . . 35

3.2 The accuracy in \% describing the estimate of number of components (NoC) over 75 trials. 35

4.1 Mean Square Error (MSE) (Percentage of Undetected Source number (PUS)), Averaged over 20 trials for three cases (One Source, Two Sources and Three sources . . . . . . . . 44

$4.2 \mathrm{SD}$ in mm Averaged over 10 trials $\ldots \ldots \ldots \ldots \ldots$

4.3 Standard Deviation of SD Error for Otsu and HR-sLORETA. One S, Two S, Three $S$, and Four $S$ illustrate using one source to four sources in simulation respectively. . . . . . . 50

$4.4 \mathrm{SD}$ in $\mathrm{mm}$ for $\mathrm{SNR}$ of $5 d B \ldots \ldots \ldots \ldots \ldots \ldots \ldots \ldots$ 


\section{List of Figures}

2.1 Neuron and its Synapse. . . . . . . . . . . . . . . . . . . . . . . . 5

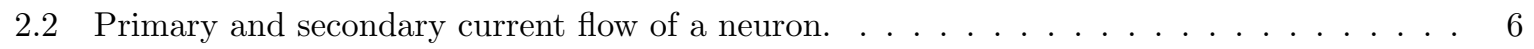

2.3 Action Potential (On the left) and excitatory/inhibitory post-synaptic potential (on the

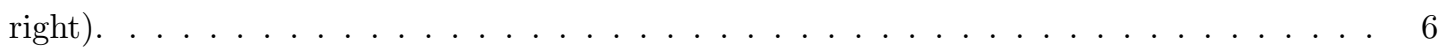

2.4 The basis of EEG measurements in Neurons. . . . . . . . . . . . . . . . . 7

2.5 The basis of MEG measurements in Neurons. . . . . . . . . . . . . . . . . 8

2.6 Coronal section of the brain with tangential and radial dipoles. $\ldots \ldots \ldots \ldots$

2.7 Supper position principle between the sources and the sensors. . . . . . . . . . . . 9

2.8 Electrode positioning: from left to right are superior, frontal, and lateral views of the brain 11

2.9 Two Dimensional (2- $D$ ) projection of the electrode placement with each channel shown with 'Letter Number' format or 'Letter Letter' format. . . . . . . . . . . . . . . . . . 11

2.10 General overview block diagram of forward and inverse modeling. . . . . . . . . . . . 17

2.11 Simplified representation of the grids projection on the surface of the head, while each source is located at the center of each grid square. . . . . . . . . . . . . . . . 19

2.12 Four layers spherical head model _ . . . . . . . . . . . . . . . . . . . 21

2.13 Illustration of how Magnetic Resonance Imaging (MRI) images are utilized to generate Boundary Element Method Boundary Element Method (BEM) model of the head. . . . . 22

2.14 An overview on Regularization based solutions . . . . . . . . . . . . . . 26

3.1 Block diagram of ICA and SSP subspace-based methods used for removal of artifacts. . . 29

3.2 A segment of MEG data with blink and non-blink events highlighted. . . . . . . . . . . 31

3.3 Simulated EEG data with blinking artefact from electrode-space for 19 channels and 30

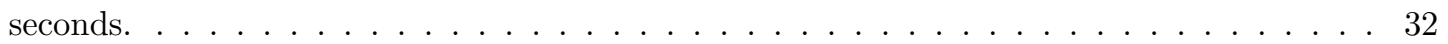

3.4 Real MEG illustrating a blinking point time data obtained from an individual using 274

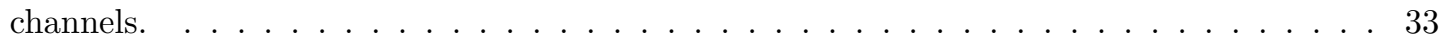

3.5 Topography of the first four components from concatenate MEG blinking artefacts . . . . 36

3.6 Comparison between EOG times series and the four first components . . . . . . . . . . 37

4.1 Existing $L_{2}$-Regularization post-processing (Thresholding) $\ldots \ldots \ldots \ldots$

4.2 Proposed automated post processing (Automated EEG Source Error Thresholding (AE-

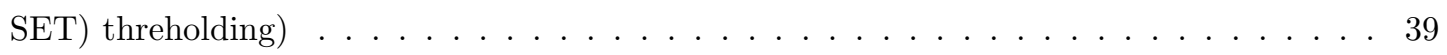


4.3 Three sources estimation evaluation for Signal-to-Noise Ratio (SNR) of 5dB. The subscript under each method illustrate the amount of thresholding in percent. . . . . . . . . 43

4.4 Illustration of Standard Low Resolution Electromagnetic Tomography (sLORETA) with various thresholds SLORETA for the case of 4 sources. . . . . . . . . . . . . . 48

4.5 Quantitative comparison of Otsu method, Higher Resolution sLORETA (HR-sLORETA), and Improved HR-sLORETA in SNR of $5 d B$ using one, two and three sources. . . . . . . 53

4.6 Illustration of (4.24) performance on two synthetic sources. . . . . . . . . . . . . 55 


\section{Chapter 1}

\section{Introduction}

\subsection{General View and Motivation}

One way to understand the behavioural activity of the brain is to analyze its electrical activity. In contrast to Magnetic Resonance Imaging (MRI) or Functional Magnetic Resonance Imaging (fMRI), which measures the hemodynamic response of the brain, Electroencephalography (EEG) and Magnetoencephalography (MEG) are two non-invasive measurements that measure the electrical activity of the brain as a fluctuation of voltage/magnetic field. The observed changes are due to the excitation of the neurons in the brain and their corresponding action potentials. The benefit of EEG and MEG in addition to being non-invasive is their high temporal resolution (in order of milliseconds) while methodologies such as FMRI provide better spatial resolution. Therefore, the problem of providing high spatial resolution through EEG/MEG while preserving high temporal resolution is a fascinating topic in the field of biomedical signal processing. The process of obtaining the spatial location of the activated source in the brain using the analysis of EEG and MEG measurements is called source localization or inverse problem. The main objective in brain source localization is to overcome the disadvantages of EEG/MEG data for having low spatial resolution. In other words, an accurate estimate about the spatial information of the brain while the temporal resolution is kept high. Achieving the same goal by increasing the rate of measurement for FMRI or similar imaging modalities is not possible by nature. This is because activities and fluctuations in the brain other than neural activity occur with a delay and at a lower rate [1]. Having simultaneous and accurate temporal and spatial information about the activities in the brain is very beneficial for many applications. One of its uses, which recently has attracted the attention of many neuroscientists and researchers, is the functional connectivity defined as the correlation between different regions of the brain. This correlation is observable only if both temporal and spatial information of neurophysiological events are available $[2,3]$. Another important application of source localization is identifying the location of the source that may cause a seizure event in an epileptic patient. Pre-surgical evaluation of the patient based on EEG/MEG data can provide valuable information about the location of the seizure. Source localization, as well as functional connectivity techniques, can provide some 
promising information in regards to prediction of the location of the seizures $[4,5]$. Providing insight and treatment on the neurological/neuropsychological disorders such as Alzheimer [6,7], depression [8, 9], schizophrenia [10, 11, 12], epilepsy [13] and even attention-deficit/hyperactivity disorder (ADHD) [14] are all other benefits of EEG and MEG source localization and processing techniques.

Due to the noise, high dimensionality of the data, and having a greater number of unknown parameters, the inverse problem still is an open problem that requires further improvement for many applications. The focus of this thesis is on the pre-processing as well as the post-processing methods, which will lead to a more efficient, higher resolution, and faster computation of EEG and MEG data, especially in the study of brain source localization. The proposed denoising methods will focus on removing the effect of "non-brain" sources such as ElectroOculoGraphic (EOG) signals (eye movement and blinking), while in the context of brain source localization, an automatic post-processing threshold is introduced that increases the resolution of brain source localization methods.

\subsection{Thesis Summary}

The focus of this thesis is on the analysis of EEG and MEG measurements. The chapters of the thesis are organized as follows:

Chapter 2 provides an overview of the background information and general assumptions of the topic. Section 2.1 includes information about the anatomy of the brain and structure of neurons, which are the basic knowledge needed for forward modelling in source localization. In Section 2.2, the biological source of EEG and MEG measurements are explained to provide the readers with some intuition on the type of data that will be processed and analyzed. Section 2.3 introduces the general assumption of all EEG and MEG data analysis and connects the biological information introduced in Section 2.1 to the structure of measurements explained in Section 2.2. The information provided in Section 2.4 helps to understand the basic pre-possessing steps that are taken while working with EEG and MEG data. This includes the step by step procedure on shaping the data in a format that can be processed as well as the technical information on the pre-pressing methodologies, which are utilized throughout the thesis. An overview of the forward model and the inverse model in brain source localization and general formulation of the problem is provided in Section 2.5, while the detailed explanation of the forward model and the inverse model are presented in Section 2.6 and Section 2.7 respectively.

Chapter 3 focuses on the pre-processing method, which is proposed for estimating the number of components of blinking artifact in subspace-based denoising methods. To be more specific, the examples of this method are be Independent Component Analysis (ICA) and Signal Space Projection (SSP). Section 3.1 is the problem formulation and its proceeding sections explain the state-of-art methods for removing ocular artifacts (Section 3.2). Moreover, the proposed approach and its implementation procedure is introduced in Section 3.3, and simulation results of the method is illustrated in Section 3.4.

Chapter 4 describes the proposed post-processing method introduced in the context of brain source

localization. The description of the group of $L_{2}$-Regularization inverse approaches and the limitation of this category of solution is provided in Section 4.1. Moreover, the proposed method, which is a post- 
processing step on $L_{2}$-Regularization inverse solution, is introduced in the following section. Section 4.2 describes the proposed method in details and illustrates the simulation and the results obtained for the method. In Section 4.3.1 and Section 4.4, two improvements on the method are introduced, which both focus on providing a better noise variance estimate. In this case, the results are provided with an experiment on the most popular $L_{2}$-Regularization inverse solution. Finally, in the last section of this chapter, by computing an example, the effect of additional constraints in the $L_{2}$-Regularization based solutions is analyzed.

Chapter 5 provides a summary and conclusion of the thesis as well as future work, which can be considered for the proposed methods.

\subsection{Publications}

- Y. Sadat-Nejad and S. Beheshti, Higher Resolution sLORETA (HR-sLORETA) in EEG Source Imaging, 41st Annual International Conference of the IEEE Engineering in Medicine and Biology Society (EMBC'19), 2019.

- Y. Sadat-Nejad and S. Beheshti, Automated EEG Source Error Thresholding (AESET) in L2Regularization inverse Problems, 16th Canadian Workshop on Information Theory (CWIT 2019), 2019 .

- Y. Sadat-Nejad and S. Beheshti, Efficient Blinking Artifact Component Estimation in SubspaceBased EEG and MEG Analysis, Asilomar Conference on Signals, Systems and Computers (ACSSC), 2019. 


\section{Chapter 2}

\section{Background on Basic Concepts and General Assumptions}

\subsection{Introduction to Brain Anatomy and Structure of Neurons}

The brain is composed of three major components: brainstem, cerebellum, and cerebrum. The brainstem connects the two other components (cerebellum and cerebrum) to the spinal cord. It is also responsible for automatic functions of the body, such as breathing, keeping the heart rate, body temperature, digestions, swallowing, etc. On the other hand, coordinating muscle movements, posture and balance as basic volunteer movements are all under the command of the cerebellum. The largest component of the brain, cerebrum, consists of two hemispheres, which are known as the left hemisphere and right hemisphere. The structure of each hemisphere is a thick layer of folded, neuronal tissue; and they are connected by a bundle of fibres called corpus callosum. The cerebrum is responsible for higher functions, which could also be defined as interpreting senses such as touch, vision and hearing. Moreover, other high-level functions that are under the responsibility of cerebrum are: control of the movement, reasoning, speech, and emotions [15]. Each hemisphere can be further divided into four lobes: frontal, temporal, material and occipital. Different regions of the lobes interact with each other in a complex form to perform a specific function. The surface of the cerebrum is called the cortex. There are roughly 16 billion neurons on the surface of the cerebrum. The cortex can be categorized into two matters, known as gray matter and white matter. Gray matter consists of grey-brown nerve cell bodies while the white matter long nerve fibres are known as axons [15].

The electrical properties and activities within every cell and tissue are known as electrophysiology. This phenomenon was first introduced by Galvani as "animal electricity" [16]. In the brain, there are specialized cells that are responsible for transmissions and process of electrical activities; these cells, which are fundamental units of the brain, are known as neurons. There are approximately $10^{10}$ neuron cells in the whole brain [17]. Neurons are usually composed of axons, myelin, body, and dendrites. Dendrites are responsible for cell-to-cell communication. The information observed by the dendrites is 
integrated by the cell body to produce an action potential. Generation of action potentials causes the release of a chemical signal in the axon called neurotransmitters. Neurotransmitters are released into the synaptic cleft, which will then be diffused to the target neuron. This will act as a messenger for the membrane potential to either get excited or inhibited. The excitation of the membrane potential is called Excitatory Post Synaptic Potential (EPSP) while inhibiting it is known as Inhibitory post synaptic potential (IPSP). Figure 2.1, part (b), illustrates two neurons which are in contact by pre and

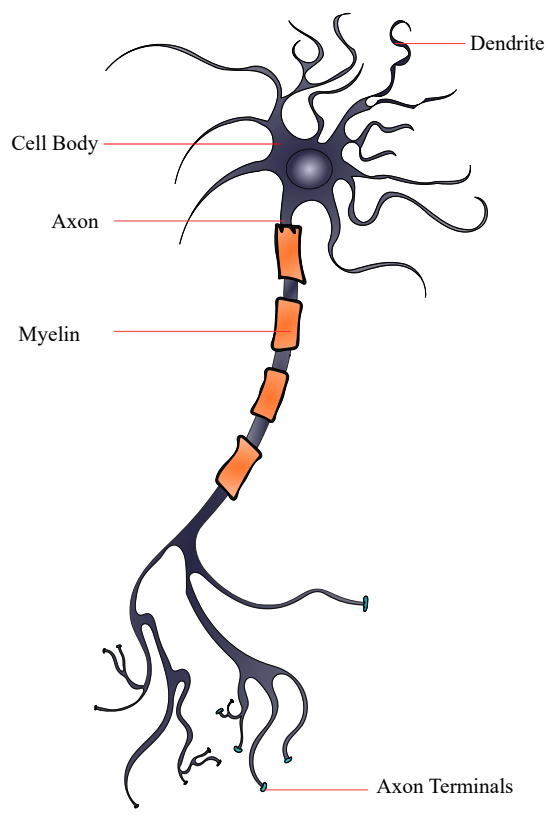

(a) A Neural cell with its body parts

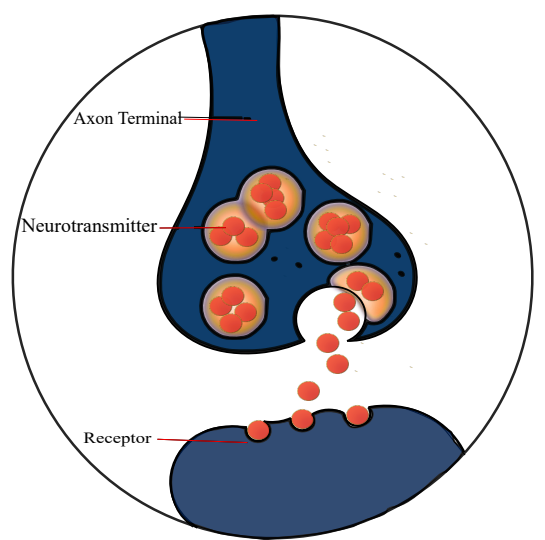

(b) Pre-syanptic, neurotransmitters and post-synapic neurons.

Figure 2.1: Neuron and its Synapse.

post-synaptic neurons. If there is a pre-synaptic action potential, neurotransmitters will be released to the post-synaptic neuron. This will cause a post-synaptic action potential in the second neuron (if the trigger is above the threshold). The resultant action potential has two important characteristics. Firstly, the time span is very short (few milliseconds), and secondly, it has bi-phasic pattern [15].

The neurotransmitter and post-synaptic action potential will act as excitatory or inhibitory message for the target neuron, which through a process, leading to the depolarization of the membrane. This depolarization causes a potential difference and flow of the current. In general, there are two types of current flow: primary current and secondary current. The primary current is referred to the majority of the current, which takes the shortest distance to flow from the cell to the sink; while the secondary current is the one which takes the longer route [15].

The time span of the excitatory or inhibitory postsynaptic potential is larger than the action potential, 


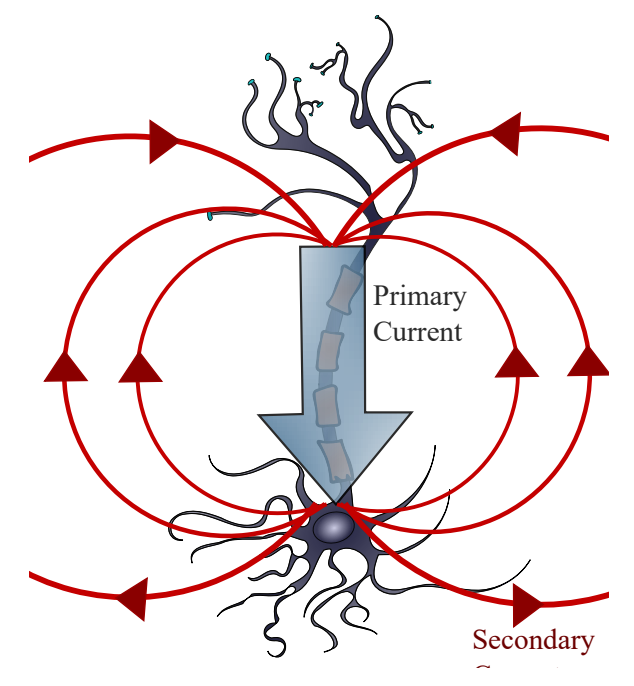

Figure 2.2: Primary and secondary current flow of a neuron.

and it is approximately ten milliseconds. Also, the postsynaptic potential has a monophonic pattern. Due to the significant distance from the surface of the brain and low conductivity of the brain tissues, observing the activity of a single neuron is not possible. Therefore, the summation of multiple neurons is observed by the sensors. Due to the biphasic characteristic of the action potential, most of the power of the action potentials in the summation may cancel out. Hence, it is more likely to observe postsynaptic potentials instead of the action potentials as the source during EEG/MEG measurements. The direction of the sources, as well as the strength of the source, is dependent on the summation of the multiple neurons [18].

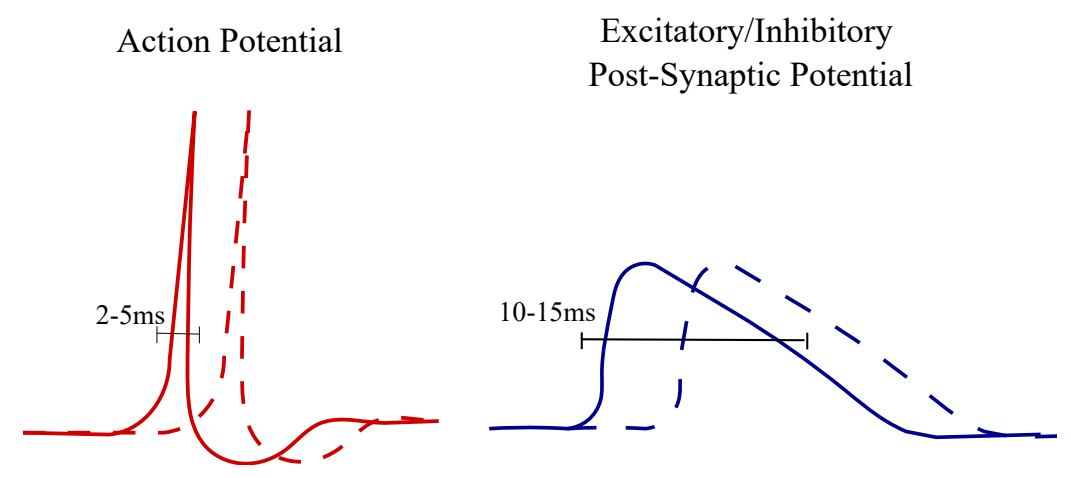

Figure 2.3: Action Potential (On the left) and excitatory/inhibitory post-synaptic potential (on the right). 


\section{$2.2 \quad$ EEG and MEG}

\subsubsection{Electroencephalography (EEG)}

EEG is a measuring system with set sensors (order of 10 to 100), responsible for measuring the electrical activity of the brain in a non-invasive manner. The measurements on each channel illustrate the electrical changes of the brain with respect to time. This is done by showing the difference between the electrical potentials of each sensor and the reference electrode. More information on how the reference is set is explained in Section 2.4.1. The data observed by EEG electrodes are known to be originally generated from the flow of the current in neurons. A single neuron is not measurable from the surface of the head, and this is due to its distance from the head's surface and low conductivity of the skull and surrounding tissues. Therefore, it is assumed that the measured potential is from the population of the neurons that are activated simultaneously.

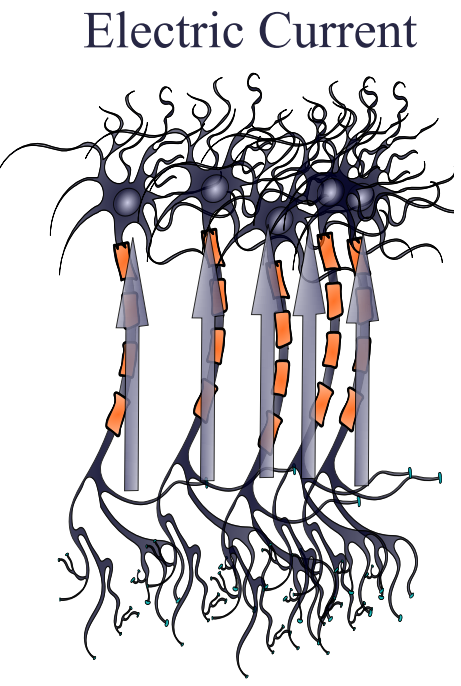

Figure 2.4: The basis of EEG measurements in Neurons.

\subsubsection{Magnetoencephalography (MEG)}

MEG measurement intends to detect the magnetic field produced by neurons in the brain. Compared to the environmental magnetic noise, these brain fields are small, and hence, sensitive detectors are required to obtain the data. In 1972, the first MEG device with Superconducting Quantum Interference Device (SQUID)-based was used, which was able to detect the magnetic field of the brain [19]. The current MEG devices use SQUID coupled with magnetometers to observe the magnetic field generated by the neurons of the brain. Using magnetometers is necessary to allow the device to observe the magnetic field from a larger area. The MEG measurement is in the frequency ranges of $10 \mathrm{mHz}$ to $1 \mathrm{KHz}$, and several picoTesla in the magnitude. This is while the urban magnetic noise goes as low as $1 \mathrm{nT}$, which is a factor of 1 million larger than MEG signals of the brain [17]. Therefore, in addition to susceptible sensors, proper shielding is also necessary for a better result. 
Figure 2.5 illustrates the poles of neurons, which generate current in the same direction. Based on the right-hand rule, with each current, there is a magnetic field associated with the direction shown in the figure. Since the direction of each neuron is same, one can illustrate field associated with the poles of the neurons as one magnetic field. This magnetic field is observed by MEG device.

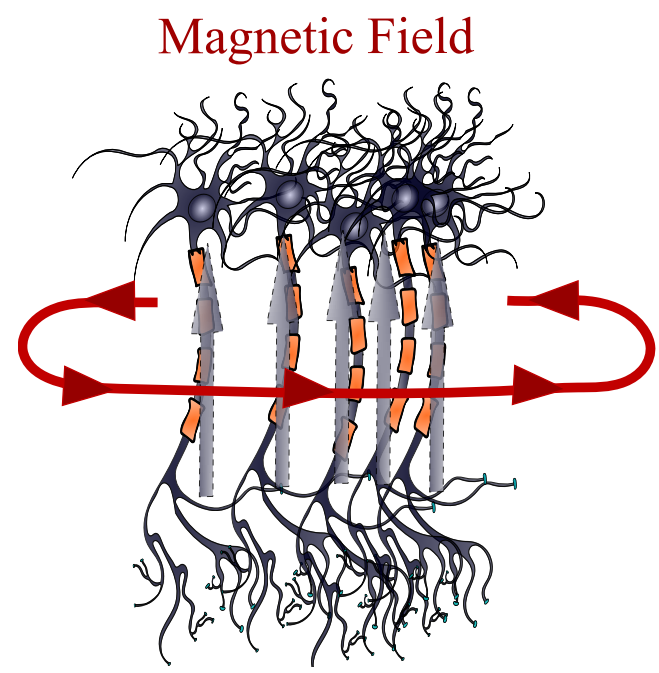

Figure 2.5: The basis of MEG measurements in Neurons.

\subsubsection{EEG and MEG Complementary}

EEG and MEG measurements are complementary. This is due to the nature of the measurements. If the current source in the brain is fully tangential, is it not observable by the EEG measurement and the magnetic field is only observable on the surface of the head. On the other hand, if the current source in the brain is purely radial, it will not produce any magnetic field outside of the head, and therefore it is only observable by the EEG measurement[17]. Figure 2.6 illustrates this concept by showing the coronal section of the human brain and two sources (dipoles). One dipole is located horizontally (tangential dipole) while the other is vertical (radial dipole). If a tangential dipole exists as a source in the brain, EEG would not be able to detect a source as such due to the placement of the dipole; on the other hand, the radial dipole would not produce any magnetic field that is observable with MEG [18]. 


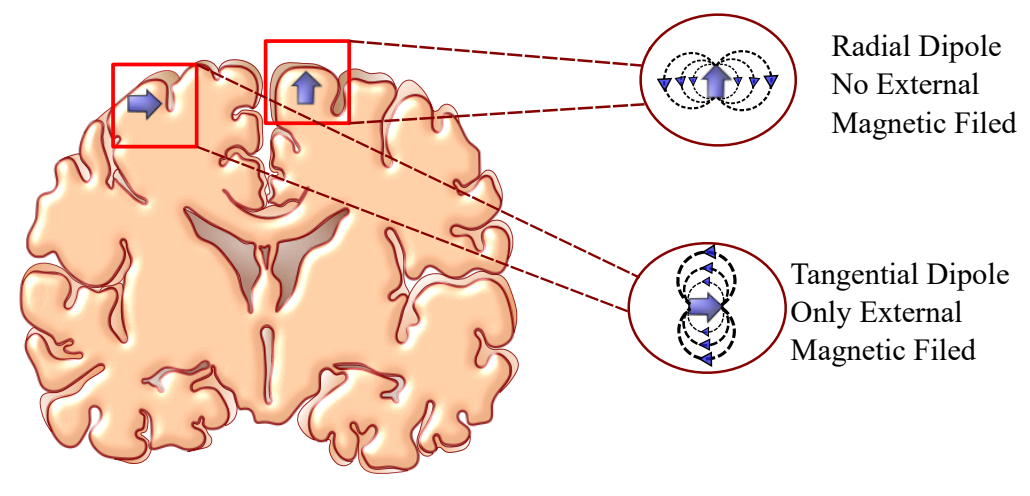

Figure 2.6: Coronal section of the brain with tangential and radial dipoles.

\subsection{Principal Supper Position}

Section 2.1 describes the biological structure of EEG and MEG data, while Section 2.2 illustrates the origin of the measured data and its source. These general concepts allow for the construction of valid assumptions that make the related problems solvable. The first and most important assumption of all brain source localization methods is the supper position of the sources. The primary assumption is that the observed potential on the surface of the brain is linear a summation of the sources in the brain. This is shown in Figure 2.7. One could see that the sources within the brain (highlighted in yellow) are projected to the surface of the head via the superposition principle. This means that the obtained signal at each electrode is a linear combination of sources as well as additive noise.

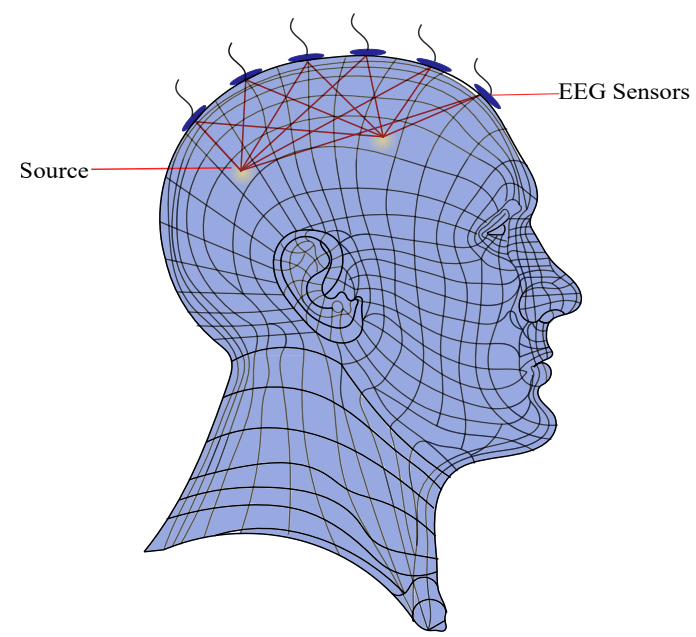

Figure 2.7: Supper position principle between the sources and the sensors. The head model is inspired from $[20]$. 


\section{4 pre-processing}

In this chapter, the focus is on general pre-processing steps applied to raw EEG and MEG data before any particular analysis, such as source localization, is applied. Processing includes all of the steps that occur between collecting the data and analyzing it [21]. These steps include some organization and ordering (such as electrode positioning) to more advanced processes such as artifact removal. First, a brief overview of basic pre-processing steps such as time sampling, electrode positioning, referencing, and frequency filtering is provided, then the chapter will focus on the subspace-based method for artifact removal known as Blind Source Separation (BSS) [22, 23, 24]. ICA and SSP as two important applications of BSS in the context of EEG/MEG signal processing are explained in details. All mentioned methods utilize the statistical characteristic of the desired data and the noise (artifacts) to separate the irrelevant components from the data. In Section 3.3, I am proposing a method that estimates the number of artifact components in the case of blinking artifacts. The method is described in details, and the following simulations and results are illustrated.

\subsubsection{Time Sampling, Electrode Positioning and referencing}

\section{Sampling}

Appropriate sampling must occur at the time of recording. While the physiological fluctuation of neural activity occurs continuously, the data must be discretized and sampled during the recording to be used in digital devices such as computers. The sampling stage involves following Nyquist criterion which states that to accurately sample data with a maximum frequency of $f_{\max }$ the sampling rate $\left(F_{s}\right)$ must be at least $2 \times f_{\max }$. This means that if $F_{s} \geq t w o \times f_{\max }$ does not hold, the used sampling rate will lead to aliasing [25].

\section{Electrode Positioning and Naming}

As explained earlier, the obtained data from EEG and MEG measurements consist of a set of channels in which each channel includes activities of the brain obtained from that specific sensor. This means that a EEG data with 19 channels includes 19 sensors which each sensor is located at a specific location on the surface of the head. Electrode positioning includes following a standard metric for the position of the sensor, depending on the number of channels, that allows anyone who obtained the data for further processing known where each channel was located. In EEG measurements, in particular, this standard is used to allow for replication of the experiment since the number of electrodes could vary a lot [26]. The standards are described as two numbers, which indicate the percentage of the distance from one electrode to others. For example, 10/20 Standard indicate that the distance between an electrode to other electrodes is $10 \%$ or $20 \%$. It is trivial that, when a higher number of electrodes is used, the distance between each electrode will be lower. Therefore, for dense EEG measurements, 10/5 standards have developed [26]. Moreover, each electrode is named with a letter (identifying the lobe) and a number or another letter that identifies the hemisphere location. The common letters used for identifying the lobe are: F, T, O,C,P which stands for Frontal, Temporal, Occipital and Parietal respectively. The odd suffix 
numbers following the mentioned letters refer to left hemisphere while even numbers are representing right hemisphere. Lastly, the letter $z$ is used as the suffix to explain the electrodes that are in the midline. This means that the $F 4$ refers to an electrode positioning on the right hemisphere of the frontal lobe, while $P z$ describes an electrode positioning the midline of parietal lobe [27]. Consider the following sketch demonstration in Figure 2.8 for a visual demonstration of electrode naming and positioning.
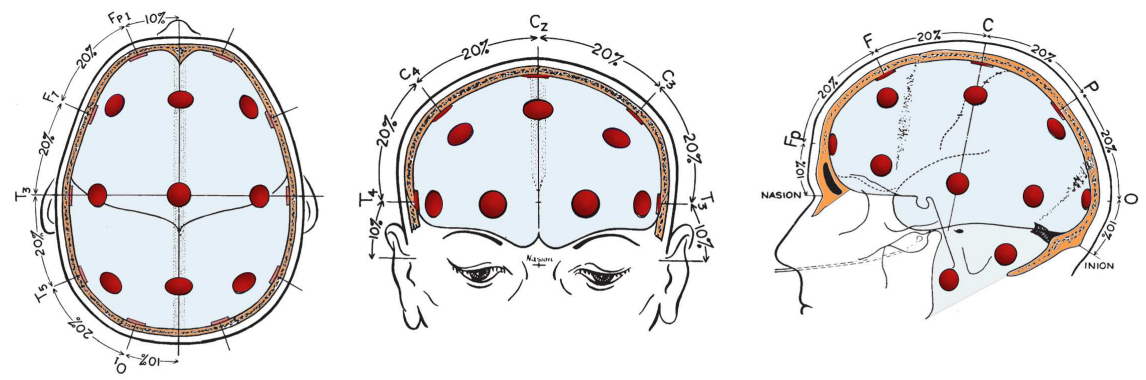

Figure 2.8: Electrode positioning: from left to right are superior, frontal, and lateral views of the brain. The figure is edited and modified version of the figure in [27].

The electrode placement is often shown with the 2- $D$ plane projection of the electrodes, as shown in Figure 2.9 [27].

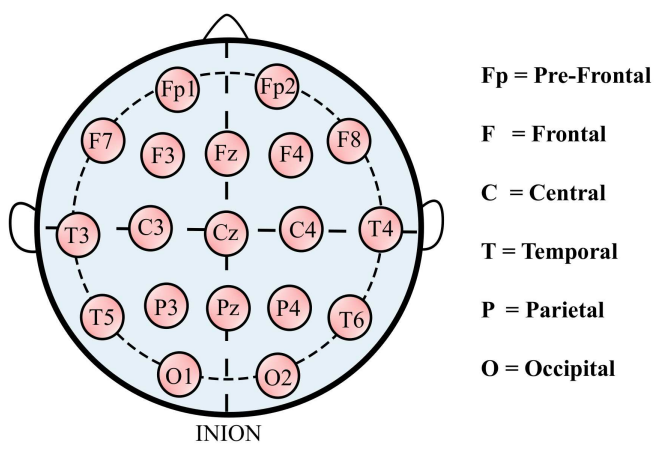

Figure 2.9: Two Dimensional (2-D) projection of the electrode placement with each channel shown with 'Letter Number' format or 'Letter Letter' format.

One of the earliest steps toward any EEG/MEG processing is registering the obtained data with the electrode channels to ensure that each channel is named and positioned correctly. Brainstorm [28] and EEGLAB [29] are two MATLAB toolboxes that provides various electrode positioning standards for different number of channels.

\section{Referencing}

While MEG is a reference-free measurement, EEG measurements require referencing. The referencing is used because the voltage values presented by each channel is relative. The placement of the reference 
electrode is critical, as a close-to-brain reference will record brain activities (which must be avoided) while far-from-electrode reference will have very different measurements and hence the signals will be very noisy. Average mastoid (bone behind the ear) and earlobe are two mainly used references EEG measurements [21]. However, both of the mentioned require an extra electrode designed and placed during the measurements. The most simple solution, when the reference electrode is not available, is to use average reference. In average reference, each measure is illustrating the difference between the channel and the average of all data. In all experiments of this thesis, the average reference is utilized.

\subsubsection{Frequency Filtering}

The observed EEG/MEG measurements always include unwanted measures and activities referred to as noise. The objective of any denoising/filtering process is to remove the noise while maintaining the data. The difficulty of maintaining this balance has led to the introduction of various denoising and processing approach. Frequency filtering is one of the most straightforward approaches used to remove high-frequency artifacts and low frequency-frequency drifts [21]. It is known that for most of the physiological and clinical tests, the activities of interest are in the range of $0-30 \mathrm{~Hz}[30]$. Therefore, a simple band-pass filter with a high-pass frequency of $0.5 \mathrm{~Hz}$ and low-pass frequency of $50 \mathrm{~Hz}$ provides an acceptable high and low-frequency filtering. Moreover, a notch-filter with the primary frequency set at $50 \mathrm{~Hz}$ or $60 \mathrm{~Hz}$ is used to attenuate electrical noise [21].

\subsubsection{Artifact Removal With Subspace Approaches}

In many cases, the artifacts are the underlying activities which are not removable by simple filtering methods introduced in Section 2.4.2. For the cases as such BSS is used. In this section, basic concepts of ICA, Principle Component Analysis (PCA), and SSP are explained.

\section{Independent Component Analysis (ICA)}

Independent Component Analysis (ICA), aims to perform a linear transformation on the data such that the obtained result includes a set non-Gaussian data (components) with each component being statistically independent [31]. In the context, of EEG/ MEG signal processing, ICA could be used to separate signals from the noise (that is assumed to have Gaussian distribution). The basic concept of ICA considers having signals which are obtained after linearly mixing statistically independent components and hence its objective is to find the linear mixture transformation matrix [31]. In the context of EEG/ MEG measurements, the assumption is that the neurological data on the cortex (components) are statistically independent and the obtained data on the surface of the head are a linear mixture of the mentioned components. For this reason, ICA have appropriate application in EEG / MEG denoising. This concept can be presented with vector-matrix notation as follows:

$$
X=A S
$$


Where $X$ is the observed data (known), $S$ are the components (unknown), and $A$ is the transformation matrix (unknown). To obtain $A$ and $S$ having the $X$, there are two primary assumptions. First is that the components $S$ are statistically independent, and second is that the mentioned components have non-Gaussian distributions [31]. For simplicity, let us assume that all components have identical distribution. To estimate one of the components (i.e. $S_{l}$ ), consider the notation in (2.2) which describes a linear combination of observed data by the vector $W$ (to be determined):

$$
Y=W^{T} X=\sum_{i} W_{i} X_{i}
$$

If the vector $W$ was a row of the inverse of $A$, then the obtained $Y=S_{l}$. Hence, the main question is how to estimate $W$ while $A$ is unknown. Moreover, to understand the concept of ICA, consider the following:

$$
Z=A^{T} W
$$

Which leads to:

$$
Y=W^{T} X=W^{T} A S=Z^{T} S
$$

Above illustrates that the constructed $Y$ by the matrix $W^{T}$ is a linear combination of $S$, where elements of $Z$ are the weights. Considering (2.4), one could see that $W^{T} X=Z^{T} S$, and obtaining the correct $W^{T}$ would lead to $Z$ having only one non-zero placed as $l^{\text {th }}$ location (This is the optimum case for $Z$ ).

By central limit theorem, it is known that $Z^{T} S$ (and hence $W^{T} X$ ), for some $Z$ that is not optimum as described above, is more Gaussian compare to $S$ itself. This prior knowledge could be used as a criterion to find $W^{T}$ that lead to $Z^{T} S$ being non-Gaussian. This criterion is known as maximizing nonGausianity for $W^{T} X$. Maximizing non-Gaussianity of $W^{T} Z$ for the described case above will lead to obtaining one of the components (i.e. $S_{l}$ ). Obtaining multi-component is also relatively easy and possible as the components are independent (and therefore uncorrelated) [31]. There are various measures for estimating Gaussianity (and non-Gaussianity) of the data, In the next paragraph most popular methods will be introduced and explained.

\section{ICA 1: Kurtosis}

Kurtosis is the fourth-order cumulant, and it is one of the measures used to find non-Gaussianity. For the standardized data obtained by z-score: $Y_{z}=\frac{Y-\bar{Y}}{\sigma_{Y}}$, where $\bar{Y}$ is mean of $Y$ and $\sigma_{Y}$ is the variance of $Y$, the kurtosis is calculated as shown in 2.5 .

$$
\operatorname{kurt}(Y)=\kappa(Y)=E\left\{Y^{4}\right\}
$$

This value, for a Gaussian random variable, is always equal to 3, and hence, any deviation from 3 illustrates non-Gaussianity. Considering the introduced notation for kurtosis, many literature such as [31], introduce a new definition of kurtosis, called excess kurtosis as: $\kappa$ (random variable $)-\kappa($ normal $)$ 
which is shown by kurt $t_{\text {excess }}$ as follow:

$$
\text { kurt }_{\text {excess }}(Y)=\kappa(Y)-3
$$

With the definition of kurtosis defined in (2.6), for non-Gaussian random variables, the value will be nonzero. Any positive value for (2.6) is called superGaussian while any negative value is called subGaussian. In practice, the matrix $W$ is first initialized with random weights; then through optimization, the weights are changed such that absolute value of (2.6) is maximized. Joint Approximation Diagonalization of Eigenmatrices (JADE) is the name of the algorithm for ICA computed based on Kurtosis. The concept is explained in Section 2.4.3. The algorithm was first proposed and developed in [32].

\section{ICA 2 : Negentropy}

Entropy measures uncertainty of a random variable [33] and it can be interpreted as the degree of information obtained from the random variable [31]. The mathematical calculation of entropy for a discrete and continuous random variable is shown, and it has proven in [33] that entropy of Gaussian variable is larger than any random variable of equal variances. Therefore, this measure could also be used for identifying non-Gaussianity.

$$
\begin{aligned}
\text { DISCRETE: } H(Y) & =-\sum_{i} P\left(Y=a_{i}\right) \log P\left(Y=a_{i}\right) \\
\text { CONTINUOUS: } H(Y) & =-\int_{i} f(Y) \log f(Y) d Y
\end{aligned}
$$

Where $a_{i}$ are the possible values of $Y$. Similar to the case of Kurtosis, the new formulation could be defined that is non-zero for non-Gaussian random variables. This formulation is called negentropy is shown as follows:

$$
J(Y)=H\left(Y_{\text {Gauss }}\right)-H(Y)
$$

Where $Y_{\text {Gauss }}$ is a Gaussian random variable with covariance equal to the covariance of $Y$. The obtained result of (2.8) is zero when $Y$ is a random variable from Gaussian distribution and non-negative for any other variable. However, the estimation of the mentioned method for negentropy is complicated, and therefore, various methods have been introduced to approximate (2.8). Maximum entropy principle [34], and higher-order moments [35] are two of the popular methods utilized to obtain approximate of negentropy.

\section{ICA 3 : Mutual Information Minimization}

The similar concept of entropy introduced above; mutual information minimization could also be used in the form of differential entropy to measure the dependency of a random variable. This concept is known as mutual information and could be used as a measure to maximize non-Gaussianity. Consider 
(2.9) that measures the mutual information between $I$ between $m$ scalar random variables:

$$
I\left(Y_{1}, Y_{2}, \cdots, Y_{m}\right)=\sum_{i=1}^{m} H\left(Y_{i}\right)-H(Y)
$$

The measure in (2.9) is always non-negative and equal to zero in the case of statistically independent random variables. Similar to previous discussions, give relation in (2.4) could be used in the form of the optimization problem to define $W$ such that (2.9) is minimized [31]. The ICA method developed based in this concept is referred as infomax.

\section{RunICA : The MATLAB Toolbox for implementing ICA on EEG and MEG data}

The concept of ICA as artefact removal method was brought to EEG and MEG signal processing by Jung et.al [36]. RunICA is a extended version ICA method, embedded in EEG and MEG signal processing toolboxes such as EEGLAB [29]. Due to the popularity of this method among MATLAB-based Toolboxes, this section is dedicated to explaining its concept.

The RUNICA algorithm, is based on the information maximization (infomax) explained in Section 2.4.3. The objective of this method is to maximize the joint entropy $H(Y)$ with respect to $W$. The proposed method in [36], explains that by using tanh function allows for the nonlinear squashing function, which provides necessary higher-order statistics for separation of sub-Gaussian and super-Gaussian components. In the proceeding section, RunICA is the other blind source separation method that will be utilized to demonstrate the capability of the proposed method.

\section{Signal Space Projection (SSP)}

Signal Source Projection (SSP) is another subspace method used in the context of EEG and MEG signal processing (mostly MEG) to denoise and filter artifacts from the signal. SSP algorithm projects the data from signal space (measurement) to source space and uses topographies (i.e spatial distribution of data at one point) to separate artefacts from the original signal. The method relies one two primary assumption : 1- Spatial distribution of the artefact is different from the source 2- External artefacts such as eye blink have low dimensional when they are projected on source space [37, 38]. For a well structured noises such as Eye Blinking (EB), subspace representation of the artefact would be as follow:

$$
\vartheta=U \vartheta+e
$$

where $U$ is a matrix (noise subspace) consist of orthonormal bases, while $\vartheta$ is $m$ component column vector with $e$ representing small error term. The major idea behind SSP algorithm is that by projecting the noisy measurement $M$ with the given orthogonal complement operator defined in (2.11), the noiseless measurement could be obtained [37, 38].

$$
P=I-U U^{T}
$$


Where $I$ is identity matrix, and $U^{T}$ is transpose of $U$ matrix. Hence simply by multiplying (projecting) the data with the matrix $P$, the denoised data will be obtained. This method will be further analyzed and utilized in Section 3.

\subsubsection{Computationally Complex Pre-Processing Methods}

It is worth mentioning that in addition to the explained frequency filtering methods, and subspace approaches, various denoising approaches exists in the filed with a similar objective of removing artifacts from EEG and MEG measurements. This includes Machine Learning [39, 40] and Deep Learning [41, 42], generic algorithms [43], and algorithms that extend subspace approaches for further improvement of the method [44]. However, the mentioned methods have high computational complexity and often not used in practice; therefore, they are not in the scope of this thesis.

\subsubsection{Whitening}

The whitening process, also known as prewhitening [45] is an important pre-processing tool used in ICA and many inverse problems. The objective of this process is to linearly transform the data into space where components are uncorrelated (i.e. data becomes white) [31]. This leads the covariance of prewhitened data equal to identity matrix [45]. One common way to obtain whitened data is to utilize Eigen Value Decomposition (EVD). Consider the covariance of the matrix $X$ defined as: $C_{x}=E\left\{X X^{T}\right\}$. In this case, EVD of the matrix is:

$$
C_{X}=U \Sigma U^{T}
$$

Where $U$ illustrates orthogonal matrix of eigenvectors and the diagonal matrix $\Sigma$ illustrates singular values $[45,31]$. With the given covariance matrix, the whitened data, shown with $\tilde{X}$, will be as follows:

$$
\tilde{X}=U \Sigma^{-1 / 2} U^{T} X
$$

where the notation of $\Sigma^{-1 / 2}$ can be computed element-wise by taking the square root of each element in the denominator [46]. This technique can be utilized as a pre-processing step, along with centring the data, for ICA method to ensure that components of the matrix $X$ are uncorrelated. Moreover, using covariance of the noise instead of covariance of the data makes the noise embedded in the matrix white noise. This concept will be utilized and explained in the section section.

\subsection{Overview of Forward Model and Inverse Model in Brain Source Localization}

Brain source localization has an objective of identifying the location of the source in the brain. However only having the EEG and MEG measurements are not enough to perform estimation as such; therefore further assumptions and knowledge about the characteristic of the source and conductivity of the different layers of the head (brain, all the tissues, scalp) are required. 
As explained in Sections 2.1 and 2.7, there are active sources in the brain (population of the neurons) that have current flow. This current flow, in the form of potential difference, is observed on the surface of the head after travelling through different volumes in the brain. The forward modelling is the study of understanding and modelling the source characteristics as well as the volume/geometry of the head. This is while inverse modelling refers to the estimation of the positions of the source having the knowledge and assumption about the source characteristics and the volume of the head.

One way to understand the forward and inverse model is by looking at Figure 2.10. In forward model, one starts with modelling the physiological source (neurons), to volume conductor for the body tissue (geometry of the head), trying to estimate the flow and distribution of the source in a way that describes the observed potential. In the inverse model, the objective is to estimate the location of the source, having the measurements as well as an estimate of the physiological models.

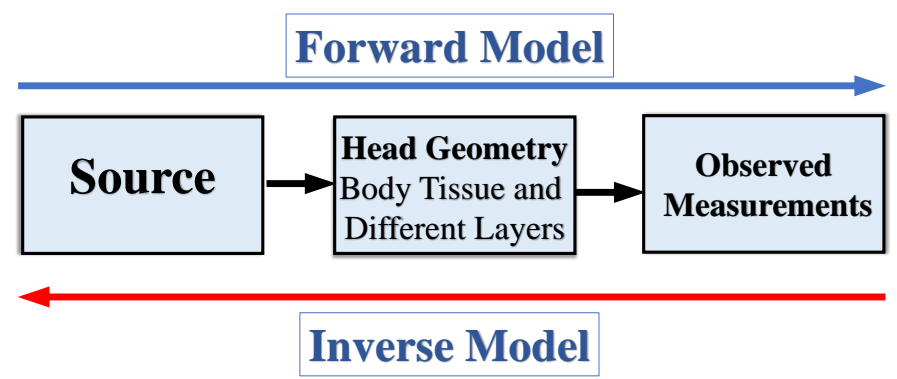

Figure 2.10: General overview block diagram of forward and inverse modeling.

While the focus of this thesis is on the Inverse problem, an introductory knowledge about the forward modelling is required. In Section 2.6, the major assumption and mathematical representation of different forward modelling will be introduced. In Section 2.7 inverse modelling, as the core concept of this thesis, will be reviewed in details.

\subsubsection{General Overview and Formulation in Brain Source Localization}

The first assumption that has to be discussed in the forward modelling is the characteristic of the source. There are two different types of modelling assumed for the source. The first type of modeling assumes that the population of the active neurons can be modelled with a number of current dipoles. This type of modelling is called Equivalent Current Dipole (ECD) modelling. The second type of modeling assumes that the sources are distributed on the surface of the cortex. The difference between the two is that in the ECD model orientation must also be considered and calculated for the sources while in the distributed source, it is assumed that all of the sources are normal to the surface of the cortex and hence the orientation is fixed [47]. There are many studies support that a distributed source model is more plausible because potential difference observed on the surface of the head is often produced by pyramidal cells that are located in the gray matter perpendicular to the surface of the cortex $[48,49,50,51]$. For this reason, this thesis focuses on methods with a distributed source model as the main assumption.

Let us assume that $N$ is the number of sensors (electrodes), and $T$ is the time with $n$ being a discrete 
sample of the time $T$. The source $(J)$ with fixed orientation in the source space is a matrix of $J \in$ $\Re^{1 D \times T}$. By the principle of superposition explained in Section 2.3, the sources will be mixed together and projected to the sensor space, which is where electrode measurement occurs. The mixing matrix, also known as lead field matrix, obtained from forward modelling, can be mathematically written as $H \in \Re^{N \times 1 D}$ for distributed source model. The matrix $H$ will be defined by using the forward model and considering spatial parameters such as head volume, the geometry of the head, the conductivity of different layers and potential location of the sources (grid resolution).

In this case, the inverse problem in the case of source localization for EEG and MEG is known to have formulation defined as a mapping from sources to the sensors [52]. This means that the relation between sources within the brain (source space) and the EEG/MEG electrodes on the scalp (sensor space) is defined with the following formulation:

$$
\bar{M}=H J
$$

where the noiseless measurement $\bar{M}$ is $N_{e}$ electrodes and $T$ time points $\left(N_{e} \times T\right)$ matrix, while the $H$ (gain matrix) that represent the forward model is $N_{e} \times N_{s}$ matrix with $N_{s}$ representing the grid points (number of potential sources in the brain). The matrix $J$ describes the active sources within the brain with the dimensionality of $N_{s} \times T$. As mentioned earlier, in the distributed source model, the objective is to estimate the amplitude of the source $J$ while the orientation is fixed to be perpendicular to the surface. The location of the sources is prefixed and defined within the matrix $H$ as a fixed grid/voxel that is on the cortex. The simplified illustration of source position and its projection on the cortex is shown in Figure 2.11. 


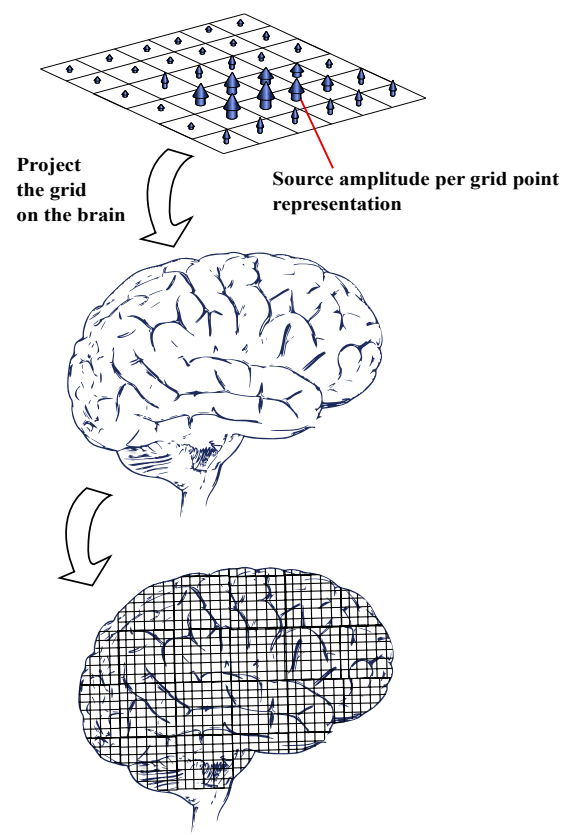

Figure 2.11: Simplified representation of the grids projection on the surface of the head, while each source is located at the center of each grid square.

Furthermore, the representation of (2.14) for each discrete point of time $n$ could be written as follows:

$$
\bar{M}(n)=H J(n)
$$

Which in the matrix form is:

$$
\left[\begin{array}{c}
M_{1}(n) \\
\vdots \\
M_{N_{e}}(n)
\end{array}\right]=\left[\begin{array}{cccc}
H_{1,1} & H_{1,2} & \ldots & H_{1, N_{s}} \\
\vdots & \ddots & & \vdots \\
H_{N_{e}, 1} & \ldots & \ldots & H_{N_{e}, N_{s}}
\end{array}\right]\left[\begin{array}{c}
J_{1}(n) \\
J_{2}(n) \\
\vdots \\
J_{N_{s}}(n)
\end{array}\right]
$$

Note that the gain matrix $H$ is fixed through the time and hence does not require to be specified with respect to time. Moreover, the matrix representation in (2.16) is shown this way to provide a sense that $N_{e} \ll N_{s}$ (i.e. the number of electrodes is much smaller than the number of source grid points which is desired to be estimated). Often the number of sensors are in the order of the 100 while the number of unknown sources shown with a grids/pixels is in order of 10, 000 which makes the solution of $J(n)$ sources underdetermined [52]. Moreover, in the non-ideal case, where noise is present due to the existence of a medium between the brain cortex and the surface of the skin (including the skull and the skin), other physiological signals (such as eye blink, heartbeat, etc.), and corruption by instrumentation 
noise during the process of measurement, the equation in (2.15) could be re-written in the form of:

$$
M(n)=H J(n)+\vartheta(n)
$$

where $M(n)$ illustrates noisy measurements and the noise is $\vartheta(n)$ which is $N_{e} \times 1$ matrix. In some cases, the noise $\vartheta$ can be further decomposed to differentiate between physiological source and instrumentation source. This can be written as:

$$
M(n)=H J(n)+\vartheta_{p}(n)+\vartheta_{i}(n)
$$

where

$\vartheta(n)=\vartheta_{p}(n)+\vartheta_{i}(n)$

and

$\vartheta_{p}(n)=$ Physiological Noise

$\vartheta_{i}(n)=$ Instrumentation Noise

Moreover, for the cases where the objective of the source localization is to locate the sources that have caused epilepsy or seizure, the formulation above must be further decomposed. In the cases as such, the background process of the brain is also considered as noise, and it is not in the interest of the experimented. Hence the equation must be written as:

$$
M(n)=H\left\{J_{e}(n)+J_{b}(n)\right\}+\vartheta(n)
$$

where

$J_{e}(n)$ corresponds to the sources that cause epilepsy.

$J_{b}(n)$ corresponds to the sources that cause the background activity of the brain.

The construction of lead-field matrix is explained in Section 2.6 and a review on the novel inverse methods will be discussed in the Section 2.7.

\subsection{Forward Model}

Forward modelling can be divided into two categories: 1- Source Modelling and 2-Head Modelling. Source modelling describes the characteristic of the source, while head modelling explains the properties of the head as a medium. These two elements of forward modelling are utilized to compute a matrix which is called lead-field matrix [52]. Lead-field matrix is the matrix introduced in the Section 2.5.1 with the notation $H$. This matrix which is also called gain matrix performs a projection of the source from the source space to the signal space.

Unlike the inverse modelling, forward modelling for a known measurement is unique. Generally, skull and other body tissues around the brain are poorly conductive, and therefore a small fraction of the current passes through the skin and skill to the EEG sensors. This fact makes forward modelling very important. Forward modelling is a difficult problem due to the in-homogeneity and ant-isotropic properties of the brain. Details description of various forward models is briefly introduced in the following section. 


\section{Volume Conduction Model}

Volume conduction model, also known as Head Model, defines geometrical and electrical properties of the head. Let's assume that the source in the brain is given with specified location, orientation, and magnitude; forward modelling provide a mathematical explanation on how the measurement observed are related to the source. In other words, head modelling explains the path that the source must travel before it is observed by the EEG/MEG measurements. Therefore, the objective is to provide a mathematical expression of the head, to explain how current flow and distribute through the brain. Volume conduction models are generally two models: 1-Analytical (Spherical) 2-Numerical (Realistic). Usually, volume conduction models are done by the use of other imaging modalities to understand the details of the brain tissues [53]. In the following section, Analytic models and Numerical Models of the forward model are described.

\section{Analytical Models}

Analytical or spherical shell model is the simplest case of head modelling. It includes one to several nested spheres for representing different layers of the brain such as scalp, skull and the cortex [54]. This model assumes that the conductivity properties of the head are uniform. This means that the model is homogeneous and isotropic $[55,56]$. It is often practical to assume different levels of conductivity of different nested spheres. As the name and description suggestions, using this model, lead-field matrix computation will be analytical and straightforward.

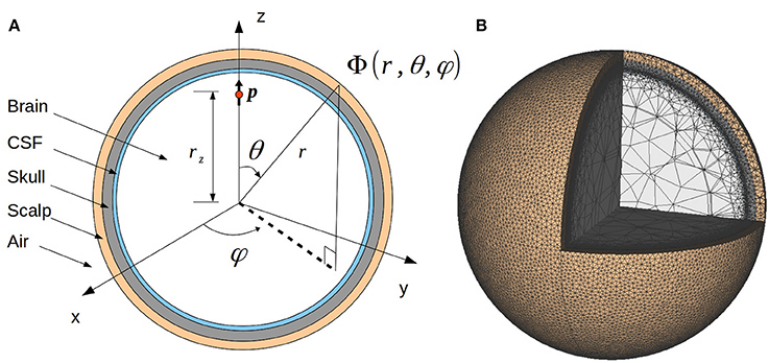

Figure 2.12: Four layers spherical head model illustrated in [57]. .

\section{Numerical Models (Realistic Head Models)}

Realistic head models are developed to enhance the accuracy of the lead-field matrix. This procedure is done by providing more detailed information about the geometry of the head as well as the conductivity of different layers. In this case, to have a more precise head model, other imaging modalities such as MRI are used to provide information about the different layers of the head. For more accurate source localization result, it is suggested to construct an individual forward model for the patient (using their MRI) [57]. In the cases where individual MRI is not available, the average of multiple MRI could be used to construct a model [28]. 


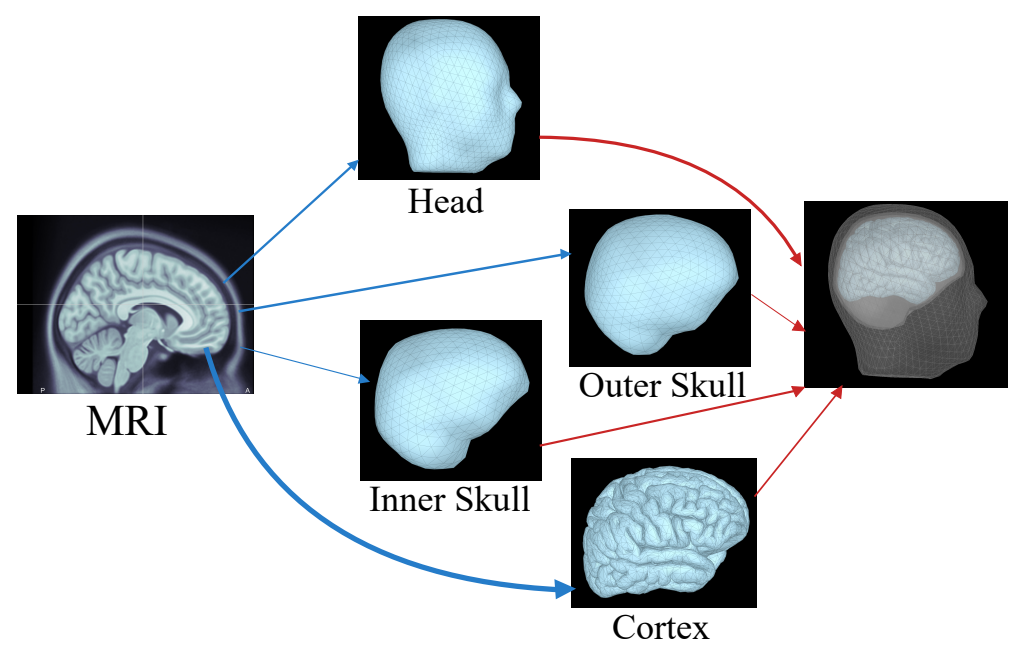

Figure 2.13: Illustration of how MRI images are utilized to generate Boundary Element Method Boundary Element Method (BEM) model of the head. The MRI and different constructed layers are obtained from BrainStorm MATLAB Toolbox [28].

Boundary Element Method (BEM) [58], Finite Element Method (FEM) [59], and Finite Difference Method (FDM) [60] are three of the most popular realistic head models used in brain source localization.

\section{Source Space}

Source space is referred as the grid or the mesh that is located on the cortex of the head. The source space represents the location of the source within the grids defining the cortex surface. In the case of the distributed source model inverse problem, the objective is estimation source amplitude at each given time sample. On the other hand, in the case of free orientation dipoles model, the orientation of the source is also considered.

Generally, there are two ways for positioning the dipoles (sources): 1-At the vertices of the mesh, 2- At the centroid of the grids [45]. Moreover, the source space could be defined in three-dimensional voxel form instead of two-dimensional grids. This means that the whole brain is considered as the source space instead of the cortex surface.

\subsection{Inverse Model}

The problem of the inverse model deals with finding the sources from the measurement. This thesis considers the inverse model with the assumption of a distributed source model, which is also known as the brain imaging method [52]. In this case, the source is presented as a current distribution of dendritic trunks of neurons with a normal orientation to the surface of the cortex. In this method, the only unknown parameter is the amplitude of the source located on each grid point; therefore, with this assumption, the inverse problem is considered linear. As mentioned earlier, the number of unknowns 
(value of source $J$ at every grid) is much higher than the number of known (electrode measurements on the surface of the head), and therefore the problem is ill-posed and under-determined. This means that, even in the noiseless environment where (2.15) holds, the estimate of the source using the pseudo inverse equation of $H$ as shown in (2.20), will lead to obtaining a non-unique solution that has localization error.

$$
\hat{J}=H^{+} M
$$

where $H^{+}=\left(H^{T} H\right)^{-1} H^{T}$ is pseudo inverse of $H$.

The derivation shown in (2.20) provides a solution which is not stable, nor unique and therefore introducing an additional constraint that makes the solution unique is required. This concept will be explained with computation in Section 4.5 .

In the paper [61], which is a complete review on EEG inverse solutions, these constraints are introduced as Hypothesis that are set for the solution. Three main group of hypothesis or constraints are: 1-Spatial, 2-Temporal and 3-Spatio-Temporal hypothesis. Various formulations have been introduced to solve for the source location while considering the additional constraints. In the next section, a brief overview of a group of solutions which are regularization-bases solutions is provided.

\subsubsection{Regularization-Based Methods}

Regularization-based methods, as a general method including $L_{2}-$ Regularization methods, are the methods that formulate the problem with the following cost function:

$$
U(J)=\|M-H J\|_{2}^{2}+\lambda f(J)
$$

where $f(J)$ is the prior assumption (additional condition) that is set to make the solution unique. The parameter $\lambda$ is used to set the trade-off between the least-square error the additional constraints. Note that (2.21) cost function is obtainable using various formulations including Bayesian formulation, error minimization, Lagrangian and Wiener filter formulation [62].

Using (2.21) formulation, various solutions have been proposed with the focus on providing the additional constraint $(f(J))$ such that the obtained solution have lower localization error and higher accuracy [61]. For instance, Minimum Norm Estimation (MNE), which was initially introduced by Hämäläinen et.at [63] focuses on the minimization of the overall intensity (energy) of the source distribution as the additional constraint [64]. In this case:

$$
f(J)=\|J\|_{2}^{2}
$$

Introducing this priori information makes the solution unique and has shown to increase the accuracy of localization. Moreover, the additional constraint in the form of $L_{2}$ least-square allows for obtaining a closed-form solution for the problem, leading to a computationally fast solution. However, the major problem with this solution is that it will suppress the deep sources and which leads to incorrect localization [65]. To compensate for this problem, one could use the information from the forward model and assign various weights for the elements of this matrix. For example, the weight matrix $\varpi$ can be defined 
as $\varpi_{i i}=\left\|H_{i}\right\|$ where $H_{i}$ is $i^{t h}$ column of $H$. This lead to the following:

$$
f(J)=\|\varpi J\|_{2}^{2}
$$

The elements of the weight matrix are known as "Depth-weight". The "Depth-Wight" is introduced to provide a higher weight of the points on the $H(r)$ that are deeper. This method is called Weighted Minimum Norm Estimate (WMNE), which is an extension of the explained MNE with additional weights as constraints. WMNE model is one of the most popular $L_{2}$-Regularization methods and will be used in Section 4.2.1, to evaluate and illustrate the proposed post-processing method. Low Resolution Electromagnetic Tomography (LORETA) is a source localization method that was introduced by Pascual-Marqui [66]. The method is called low-resolution electromagnetic tomography because the spatial resolution is relatively low while time resolution is improved. LORETA is a minimum norm estimation solution but with Laplacian and weighted current source. Low spatial resolution is due to the imposed spatial smoothness constraint, which is expressed by the use of a three-dimensional discretized Laplacian matrix. The primary assumption of this method is that neighbouring grids/voxels have similar electrical activity. The regularization term in this formulation has the following notation:

$$
f(J)=\|B \varpi J(n)\|^{2}
$$

As shown in (2.24), compare to WMNE, the parameter $B$ is the additional element included, which corresponds to discrete Laplacian operator [66]. LORETA method could be considered as $W M N E$ method in a transformed domain [61]. The objective of the additional constraint $B$ is to provide a smoother result as the inverse solution. The method Dynamic Statistical Parametric Mapping (dSPM), proposed in [67], provides the basic idea of normalizing the source estimate with respect to the variance of the noise. The objective of this approach is to decrease the influence of the presence of noise on the observed estimate. The paper [67] shows that the covariance of the noise $C$ could be used to estimate the noise itself. Using the noise variance, or source variance to normalize the solution of the inverse problem is the main idea behind this approach. Pascual-Marqui initially introduced Standard Low Resolution Electromagnetic Tomography (sLORETA) in 2002 [68] as an extension of LORETA [66], which constructs the inverse problem with the weighted-Laplacian condition. However, localization inference is based on images of standardized current density. This means that the main emphasis of SLORETA and its core concept is standardizing the solution of any given source estimate with closed-form expression [68]. Therefore, in practice, the method is called SLORETA, even if the Laplacian matrix is embedded in the original equation. This method could be considered as the most popular method in linear inverse modelling of EEG and MEG measurements, due to its simplicity and high accuracy. The benefit of this method is that it has zero localization error for noise-less measurements. This is due to the fact that the biological variance of the actual source is also taken into account in addition to the variance of the noise (compare to DSPM method). The assumption is that the biological variance is uniform across the brain. [68], and hence the method is standardized (normalized) with respect inverse operator. Consider the 
definition in (2.25), which is standardization of the source estimate for the case of EEG measurements:

$$
\left[\hat{J}_{\text {sLORETA }}\right]_{l}=\frac{[\hat{J}]_{l}}{\left[S_{\hat{J}}\right]_{l l}}
$$

where $\hat{J}_{l}$ is the current density estimate (source estimate) at the grid point $l$ and $\left[S_{\hat{J}}\right]_{l l}$ is $l^{\text {th }}$ diagonal element of the matrix $S_{\hat{J}}$. The notation $\left[\hat{J}_{\text {sLORETA }}\right]_{l}$ is the result of sLORETA source estimate after standardizing the source estimate with respect to $S_{\hat{J}}$. The matrix $S_{\hat{J}}$ is described next.

In all mentioned methods here, the additional constraints $(f(J))$ is in form of $L_{2}-$ regularization, which allows for obtaining a closed-form solution for cost function, such that for any point of time, the source estimate is as follows:

$$
\hat{J}=W M
$$

where $W$ is a kernel operator illustrating the closed-form model for $L_{2}$-based cost function.

Now consider (2.15) that illustrates the noiseless model, the general expression of the inverse problem is the equation $M=H J$. Substituting (2.15) into (2.26) will provide:

$$
\hat{J}=W H J
$$

Let $\left[S_{\hat{J}}\right]=W H$ :

$$
\hat{J}=\left[S_{\hat{J}}\right] J
$$

The Equation shown in (2.28) means that the relation between the estimation of the source $\hat{J}$ and the true source $J$ is through the matrix $\left[S_{\hat{J}}\right]$. The expression above shows that the true source ( source estimation with zero error in case of no additive noise) is derived by (2.25). The derived standardization equation allows for the computation of zero-error source estimate in the case of noise-free measurements. This method is the primary focus of the thesis, due to its popularity among linear inverse models.

The mentioned solutions above are considered as prevalent methods of source imaging due to their acceptable source estimate and fast computation. However, they generally provide a blurred solution. One category of solutions proposed to compensate for this limitation was the introduction of an additional constraint on $f(J)$ in the form of $L_{p}$-norm problem. This is written in the form of:

$$
f(J)=\|W J\|_{p}
$$

where $0 \geq p \leq 1$. While this allows for more sparse solution [69], the computational time of the solution increases as the equation does not have a closed-form solution anymore. Minimum Current Estimate (MCE) is an algorithm with $p=1$ leading to convex optimization and assumption for having fixed orientation [70]. Focal under determined system solution (FOCUSS) is another method proposed in [71], which updates the weights through an iterative process using $L_{0}$-norm. This leads to gradually shrinking the source estimate and resulting in sparse solution [61]. Furthermore, more than one constraint in the transform domain, $T$ could be used to obtain a new source estimate. For example, a sparsity on spatial domain and smoothness on the temporal domain could be combined in the form of $L_{1}, L_{2}$ solution to provide a new approximation for source estimate [61]. Mixed-Norm Estimate (MxNE) is an example 




Figure 2.14: An overview on Regularization based solutions [45].

of the algorithm with such constraints [72]. Figure 2.14 illustrates an overview of various least-square algorithms.

\subsubsection{Computationally Complex Inverse Solutions}

Other than the regularization-based solutions which relatively have simpler formulation, there exist various solutions for an inverse problem which have higher complexities with higher computational time. Among them are Tensor-Based solutions that consider additional dimensions for the data (other time space and time) and utilize the information obtained from these additional spaces to compute source estimate. The proposed methods under this category often focus on possible variations for these additional dimensions. Space-Time-Frequency (STF), which provides Short Time Fourier Transform (STFT) in the space domain as the third dimension [73] and Space-Time-Wave-Vector (STWV), which provides Wavelet transform in the space domain [74] are examples of this category. The Bayesian framework is another general framework, which looks at the source localization from the probabilistic approach. All of the methods in this category are focused on finding the fittest prior assumptions that make the solution of the problem more universal and accurate. Maximum a Posterior Estimation, Variational Bayesian (VB), and Emperical Bayesian (EmpB) are three classes of this category. Considering Bayesian general formulation, methods such as MNE, WMNE, MCE and MxNE could all be considered as derivation of maximum a posterior estimation where the prior $(p(J))$ is fixed. However, the formulation can get 
more complicated by introducing additional levels into the hierarchical model with more parameters to be considered. While in some cases, these methods illustrate better performance over the conventional methods, due to their complexity and high computational cost, they are practically not beneficial and therefore, are considered out of the scope of this thesis.

\subsection{Signal Denoising and Best Basis Selection}

This thesis is heavily based on the proposed denoising approach in [75] and its subspace Singular Value Decomposition (SVD) based approach in [76]. Therefore, this part of the thesis is dedicated to introducing the approach in [75], while the applications of the methods are explained in details in Chapter 3 and Chapter 4.

Consider $y(n)$ to be a noisy signal at sample $n$, which is constructed from noise-less data $\bar{y}(n)$ and additive noise $\omega(n)$ as shown in 2.30

$$
y(n)=\bar{y}(n)+\omega
$$

Further more, assume that (2.30) is in a subspace where, sorting $y(n)$ in descending order and selecting its first $S m$ components would provide us the denoised data $\bar{y}(n)$ (condition of such subspace and its proof is provided in [75]). In this case, obtaining the denoised version of $y(n)$ shown with $\hat{y}(n)$ is achievable if $S m^{*}$ as the optimum index of the ordered data is selected correctly from all possible $S m$ values. The method, analyze this case by considering Data Error, and Reconstruction Error describe below:

$$
\begin{gathered}
\text { Data error: } X_{S m}=\frac{1}{N}\left\|y-\hat{y}_{S m}\right\|_{2}^{2} \\
\text { Reconstruction error: } Z_{S m} \frac{1}{N}\left\|\bar{y}-\hat{y}_{S m}\right\|_{2}^{2}
\end{gathered}
$$

where $N$ is the length of the data.

The optimum index in ordered data, $S m^{*}$ is achieveable by minimizing (2.8).

$$
S m^{*}=\arg \min _{S m} Z_{S m}
$$

While this error is unavailable, it is proven in [75], that the probabilistic upper bound of (2.8), shown with $\bar{Z}_{S} m$ is achievable by utilizing (2.32), noise variance estimate, confidence probability, and criterion of Description Length (DL).

$$
S m^{*}=\arg \min _{S m} \bar{Z}_{S m}
$$

The finalized derivation, as well my proposed simplification is presented in Appendix A, while the method is used in Chapter 4. Moreover, the similar approach is utilized in [76] for selection of number of eigenvalues. This method could be considered as a branch of [75] method. The application of this method is presented in Chapter 3. 


\section{Chapter 3}

\section{Reliable Automated pre-processing}

This chapter focuses on a proposed pre-processing method that provides a reliable and automated number of component estimation for the purpose of sub-space based artifact removal. The proposed method improves the efficiency of existing subspace methods such as ICA and SSP by providing an accurate estimate on the number of components that are required to be removed.

\subsection{Ambiguities of Independent Component Analysis (ICA) and Signal Space Projection(SSP)}

The main problem in the mentioned subspace artifact removal methods is that the order of the independent components is unknown [31]. Recall that in (2.1), both $A$ and $S$ are unknown and therefore any order (dimensions) for the mentioned data can be chosen. This cause ambiguity and selection of the correct number of components becomes an important question in the pre-processing step. Selecting a high number of components and removing additional components from the data, leads to losing important information from the measurements; while selecting a low number of components leads to not obtaining the optimum demonizing result. In this chapter, a method is proposed for the automatic selection of the number of components in the case of artifact removal. The proposed method is explained and illustrated through the process of blink artifact removal, which is one of the common existing artifacts in EEG and MEG measurements.

\subsection{Ocular Artifact (OA) Removal by Subspace Methods}

Both EEG and MEG methods are often contaminated with additional unwanted components known as artifacts. One of the most prominent artifacts is an Ocular Artifact (OA) which includes EB and other low-frequency pattern artifacts caused by eye movements [77]. The difficulty of removing unwanted components while maintaining the original underlying signal, and its importance in many applications such as BCI and neuroscience researches [78], has caused a great deal of focus on developing algorithms 
in the context of artifact removal from EEG /MEG measurements [78]. Among the existing algorithms, subspace-based approaches [79] such as Independent Component Analysis (ICA) [46] and Signal Space Projection (SSP) [37] are well known and widely used due to their relative efficiency. These algorithms rely on the decomposition of the data and the separation of relevant components from artifacts. To perform such algorithms, pre-knowledge about the number of components involved in the artifact is required, which is often a user-dependent process and difficult to obtain. Here, a method of estimation of the number of artifact components using Mean Square Eigenvalue Error (MSEE) [76] is proposed. This estimate allows the subspace-based algorithm to provide robust EB artifact removal.

\subsection{Eye Blink (EB) Number of Components (NoC) Estimate as Application of Number of Source Eigenvalue Error NoSEE}

Subspace-based methods use topographies (i.e. the spatial distribution of data at one point) to separate artifacts from the original signal. These methods rely on two primary assumptions: 1- Spatial distribution of the artifact is different from the source 2- External artifacts such as eye blink have low dimensionality $[37,38]$. Subspace-based algorithms such as ICA and SSP first project the neural data $M$ into subspace (also known as component space), then the number of components corresponding to artifacts is estimated and removed. Lastly, non-artifact components are transformed back to their original space to represent the data. The block diagrams in Figure 3.1 illustrates a high-level overview on ICA and SSP artifact removal procedures, where $\hat{M}$ the EEG /MEG data when artifacts are removed. Regardless of the chosen algorithm, finding the number of artifact components (shown with $m$ ) is an important necessary procedure.

\section{A. ICA}

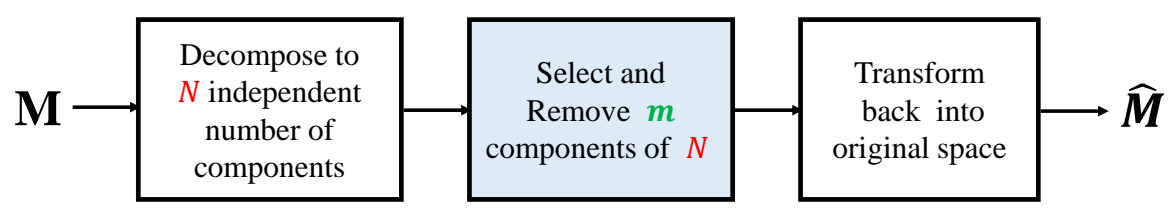

\section{B. SSP}

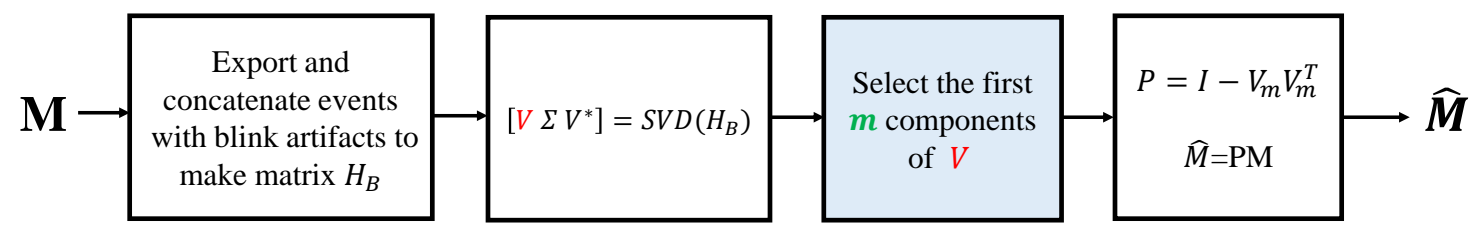

Figure 3.1: Block diagram of ICA and SSP subspace-based methods used for removal of artifacts.

Selecting the number of components $(m)$ is usually done manually and is a user-dependent process. Additionally, selecting the correct number of components requires expertise and knowledge with extensive 
analysis of the spatial and temporal character of the data (an example will be shown in Section 3.4.5). Here an algorithm that would estimate the optimum number of components $\hat{m}$ for subspace-based algorithms such as ICA and SSP is proposed. Here a brief explanation of the proposed algorithm is provided.

Let us assume that our signal of interest is the blinking artifacts while the EEG/MEG data are the unwanted signals. This allows us to re-write the general equation as follow:

$$
\Omega=\bar{\Omega}+v
$$

where $\Omega$ is pure blinking signals and $v$ is representing the EEG/MEG additive data. The objective is to find the number of components in $\Omega$, which is similar to the Number of Signal Source Estimate (NoSS) proposed in [76]. The proposed method in [76] uses the covariance matrix of the noisy signal of interest (in here matrix $\Omega$ ) as shown in (3.2), to estimate the optimum number of eigenvalues by mean square eigenvalue error (MSEE) estimation. While the original equations and derivations could be found in [76], in here, they are presented with a new notation, and with a new perspective which considers EEG and $\{$ GlsMEG as data, and their corresponding artifact as noise.

$$
C_{\Omega}=\frac{1}{N} \sum_{n=1}^{N} E\left(\Omega(n) \Omega^{T}(n)\right)=C_{\bar{\Omega}}+\sigma^{2} I_{P \times P}=U \sigma_{\Omega} U^{*}
$$

where $N$ is length of the data, $\sigma^{2}$ is variance of the noise and $I$ is Identity matrix. Additionally $\sigma_{\Omega}$ is $P \times P$ matrix of eigenvalues and $U$ illustrates eigenvectors. Moreover $C_{\bar{\Omega}}$ could be decomposed to:

$$
C_{\bar{\Omega}}=U \sigma_{\bar{\Omega}} U^{*}
$$

As described in [76], the objective is to choose the optimum $m$ largest values of $\sigma$, denoted by $\sigma_{\Omega}(m)$, such that the following (MSEE) in (3.4) minimized:

$$
Z_{m}=\left\|\sigma_{\bar{\Omega}}-\sigma_{\Omega}(m)\right\|^{2}
$$

While $Z_{m}$ is unavailable due to unavailability of noiseless eigenvalues shown with $\sigma_{\bar{\Omega}}$, In [76] the following $X_{m}$ available eigenvalue error

$$
X_{m}=\left\|\sigma_{\Omega}-\sigma_{\Omega}(m)\right\|^{2}
$$

is used to provide probabilistic bounds on the unavailable $Z_{m}$. It is shown in [76] that minimizing the upper bound of $Z_{m}$ [76], denoted by $\bar{Z}_{m}$, leads to the optimum number of components:

$$
\hat{m}=\arg \min _{m} \bar{Z}_{m}
$$

However, to use the formulation above with real MEG/EEG data, $v$ is required to be White Gaussian Noise (WGN) distribution [76]. This is ensured by prewhitening all data by use of non-blinking events and utilizing the resultant data as the input for the proposed algorithm. The details prewhitenningprocess 
areexplained in Section 2.4.5. However, the step-by-step procedure of the proposed algorithm is provided in Section 3.3.1.

\subsubsection{Step-By-Step Procedure of The Proposed NoSEE application}

Step 1: Highlight and separate MEG/EEG data into blink and non-blink events (As shown in Figure $3.2)$.

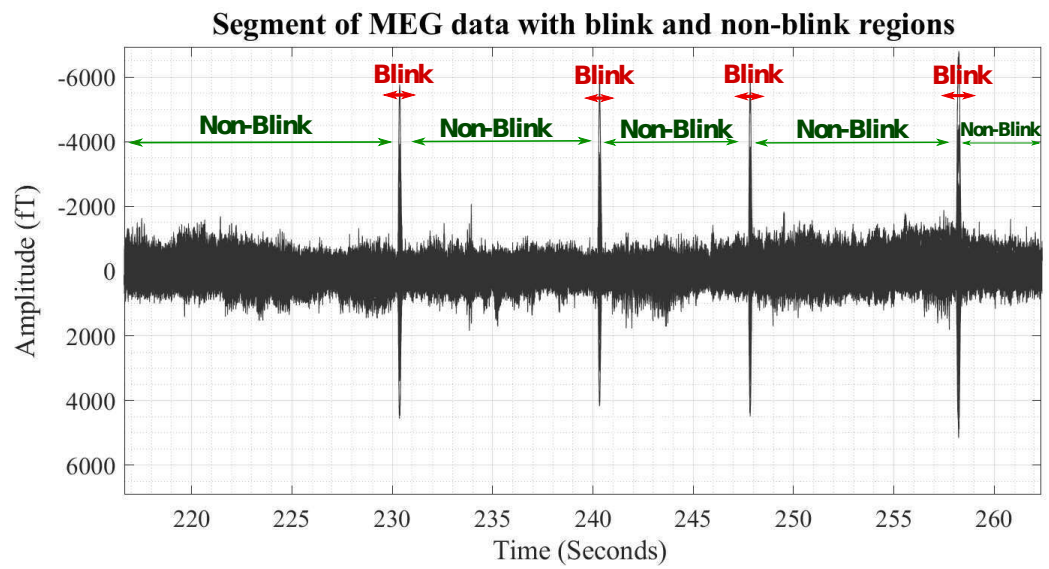

Figure 3.2: A segment of MEG data with blink and non-blink events highlighted.

Step 2: Export "Non-Blink" regions and concatenate them into a matrix called $W_{B}$.

Step 3: Obtain prewhitening operator using covariance matrix of $W_{B}$ and one of the known methods such as EigenValue Decomposition (EVD).

$$
F=U_{s_{1}} \Sigma_{s_{1}}
$$

where

$$
C_{W_{B}}=\left[\begin{array}{ll}
U_{s_{1}} & U_{s_{2}}
\end{array}\right]\left[\begin{array}{cc}
\Sigma_{s_{1}}^{2} & 0 \\
0 & \Sigma_{s_{2}}^{2}
\end{array}\right]\left[\begin{array}{l}
U_{s_{1}}^{T} \\
U_{s_{2}}^{T}
\end{array}\right]
$$

where $U_{s_{1}}$ and $U_{s_{2}}$ are eigenvectors and $\Sigma_{s_{1}}^{2}$ and $\Sigma_{s_{2}}^{2}$ are eigenvalues respectively [45].

Step 4: Perform prewhitening on the (3.1) as follows:

$$
\tilde{\Omega}=F^{+} \Omega
$$

where $F^{+}$is representing Moore-Penrose pseudo inverse of $F$ [45].

Step 5: Use $\tilde{\Omega}$ instead of $\Omega$ in (3.2) and follow the rest of the proposed method to find the number of components for blinking artifacts. 


\subsection{Simulation Results of the Proposed pre-processing Method}

Evaluating artifact removal algorithms for neural data is a challenging task, as free-artifact neural signals are not available [80]. To ensure that the proposed number of artifact component estimates is useful, both synthetic and real data have been used to evaluate the performance and validate the method. The description of both are provided in the following sections.

\subsubsection{Synthetic EEG Data for the Proposed pre-processing Method}

The synthetic EEG data is utilized for numeric evaluation and performance of the proposed method. As suggested in [81], the noise-like behaviour of EEG data allows us to represent its activities by simulating it as pink noise. After generating EEG-like background activities as pink noise, a set of blinking signals is added to the EEG data. Blinking signal activities were simulated to have large amplitude spikes (about 10 times larger than EEG background activities) [81, 82] and last for $100-400 \mathrm{~ms}$ [82] with interval repetition of 5 seconds [83]. To ensure that blinking spikes are distributed between channels similar manner to real EEG data, sinusoidal signals with different frequencies [84] (representing source space data) were located as dipoles near frontal sensors where blinking artifacts are often measured [85]. Then electrode space representation of data is obtained by projection of the dipoles using theSpherical head model provided in [86]. Figure 3.3 illustrates synthetic EEG generated for 19 channel signals with the samplingfrequency of $250 \mathrm{~Hz}$ and time duration of 30 seconds. The randomly generated pink noise as the

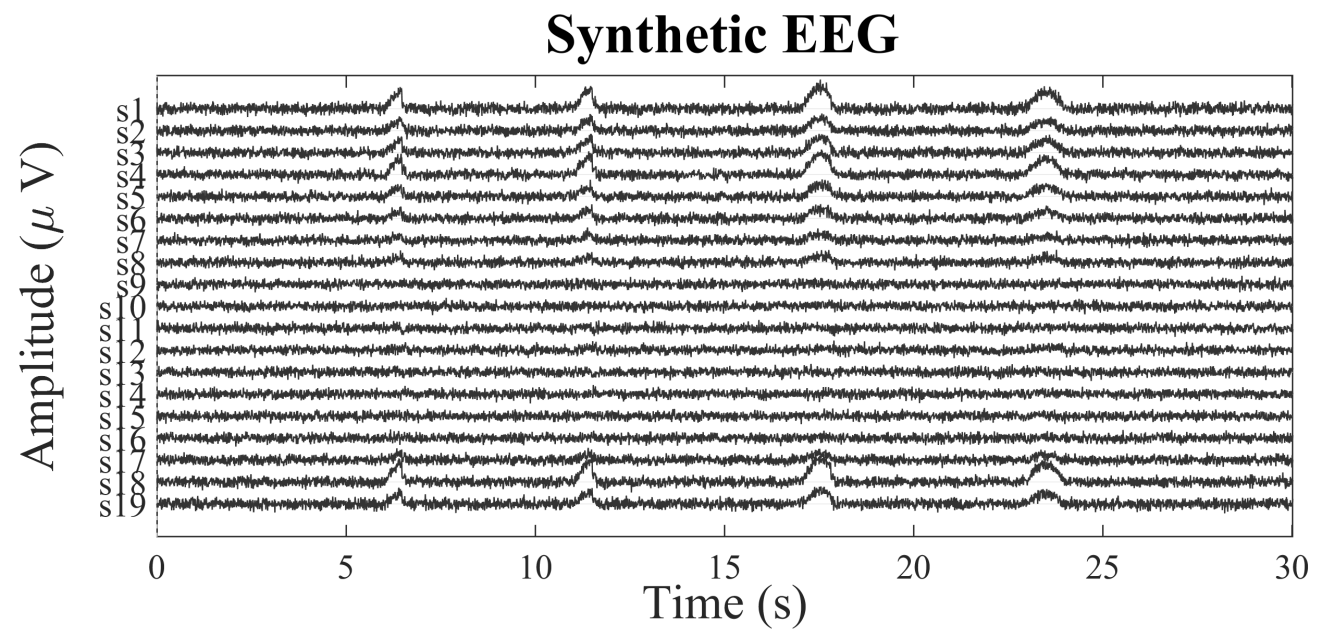

Figure 3.3: Simulated EEG data with blinking artefact from electrode-space for 19 channels and 30 seconds.

background activity was varied for every simulation. Additionally, the number of blinks, amplitude, and frequency of the blink simulations were changed, such as the true number of component changes. This was repeated 75 times to examine the liability and stability of the method. SSP projection, extendedICA [87] known as runICA (explained in the Section 2.4.3), and Joint Approximation Diagonalization of Eigenmatrices (JADE) [32] (explained in the Section 2.4.3) were used along with the proposed number 
of the componentestimate to perform artefact removal analysis. This will be further explained in Section 3.4.4.

\subsubsection{Real MEG Data}

To validate the proposed algorithm, publicly available MEG data provided in [88] is used. The data contain 6 minutes of one subject data acquisition. MEG data were obtained at a sampling frequency of $2400 \mathrm{~Hz}$ and down-sampled to $600 \mathrm{~Hz}$ with CTF 275 system. A totalof $274 \mathrm{MEG}$ channels were used to collect the data. The procedure explained in [88] was performed as a pre-processing step. SSP method explained earlier was used to perform Eye blink artifacts removal. Figure 3.4 illustrates the time series MEG data for a time point where blinking has occurred for all 274 channels. In total, 15

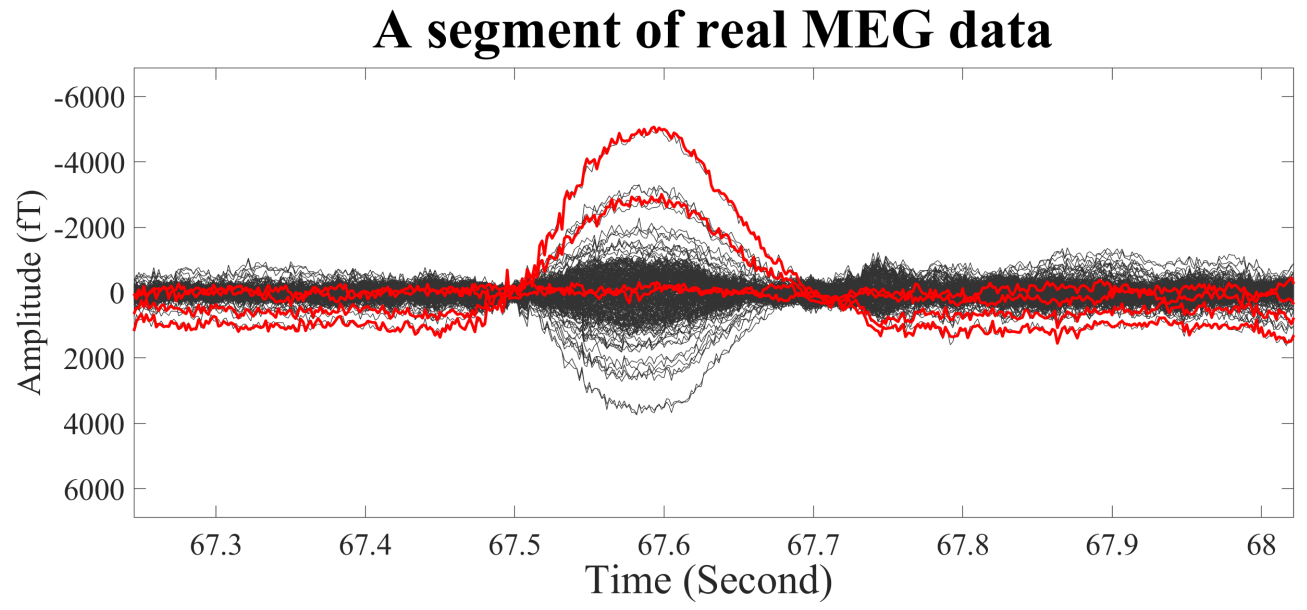

Figure 3.4: Real MEG illustrating a blinking point time data obtained from an individual using 274 channels. Plot obtained from Brainstorm Toolbox [28].

time events where blinking has occurred is marked each with a duration of approximately 3 seconds. All non-blinking events were concatenated together to make the prewhitening matrix, while all blinking events were contacted for SSP projection. The 6 minutes data was prewhitenned, and the obtained result was fed to the proposed Number of Source Eigenvalue Error (NoSEE) algorithm for blinking artifact number of component estimation.

\subsubsection{Evaluation Criteria of the Proposed pre-processing Method}

To evaluate the performance of the proposed method on the synthetic EEG data, Root Mean Square Error (RMSE) [89] is utilized. This error illustrates the normalized difference between the noise-less EEG and the obtained EEG after performing blink artifact removal. The lower value of RMSE illustrates the 
better performance of the algorithm.

$$
R M S E=\sqrt{\frac{1}{N} \sum_{i=1}^{N}(\bar{y}(i)-\hat{y}(i))^{2}}
$$

where $\bar{y}(i)$ illustrates artifact-free simulated EEG (generated pink noise) at a discretepoint $i, \hat{y}(i)$ is the data obtained after removing the blink artifacts, and $N$ is the length of the data. Moreover, validation of real MEG data was done by performing extensive manual analysis on the SSP components. The manifested analysis is following [88], which is a standard analysis that is done for manual estimation of the number of components.

\subsubsection{Synthetic EEG Result and Discussion for the Proposed pre-processing Method}

After performing the proposed method (NoSEE) on the synthetic EEG data, the obtained Number of Component (NoC) was compared with 1, 2, 3 and 4 components, which were selected manually. As described earlier, two ICA and one SSP method was used to perform artifact removal on the data, which demonstrate the efficiency of the proposed method for various subspace-based algorithms. Table 3.1 illustrates the obtained RMSE for 5 randomly selected trials out of the 75 trials. The column NoSEE shows the RMSE obtained after using NoSEE algorithm and the number in the bracket represents the chosen number of the component by the algorithm. 
Table 3.1: RMSE of SSP, runICA and JADE artifact removals . $C(i)$ represents choosing the first $i$ components. Last column illustrates RMSE as well as the optimum chosen NoC by the proposed method (in parenthesis). Last row is the averaged results over 75 trials.

\begin{tabular}{|c|c|c|c|c|c|}
\hline SSP & C (1) & $\mathrm{C}(2)$ & $\mathrm{C}(3)$ & C (4) & NoSEE \\
\hline Trial 1 & 0.00495 & 0.00438 & 0.00467 & 0.00964 & $\mathbf{0 . 0 0 4 3 8}(2)$ \\
\hline Trial 2 & 0.00498 & 0.00435 & 0.00462 & 0.00927 & $0.00435(2)$ \\
\hline Trial 3 & 0.00424 & 0.00372 & 0.00924 & 0.01006 & $0.00372(1)$ \\
\hline Trial 4 & 0.00679 & 0.00608 & 0.00462 & 0.00994 & $0.00462(3)$ \\
\hline Trial 5 & 0.00671 & 0.00618 & 0.00446 & 0.00773 & $0.00446(3)$ \\
\hline Average & 0.00516 & 0.00456 & 0.00554 & 0.01032 & 0.00442 \\
\hline runICA & C (1) & $\mathrm{C}(2)$ & C (3) & C (4) & NoSEE \\
\hline Trial 1 & 0.00488 & 0.00437 & 0.00520 & 0.00567 & $\mathbf{0 . 0 0 4 3 7}(2)$ \\
\hline Trial 2 & 0.00426 & 0.00695 & 0.00813 & 0.00918 & $0.00426(2)$ \\
\hline Trial 3 & 0.00423 & 0.00447 & 0.00732 & 0.00868 & $0.00423(1)$ \\
\hline Trial 4 & 0.00674 & 0.00618 & 0.00565 & 0.01784 & $0.00565(3)$ \\
\hline Trial 5 & 0.00663 & 0.00608 & 0.00541 & 0.00564 & $0.00541(3)$ \\
\hline Average & 0.00511 & 0.00490 & 0.00603 & 0.00797 & 0.00463 \\
\hline JADE & C (1) & $\mathrm{C}(2)$ & C (3) & C (4) & NoSEE \\
\hline Trial 1 & 0.00414 & 0.00487 & 0.00584 & 0.00815 & $\mathbf{0 . 0 0 4 1 4}(2)$ \\
\hline Trial 2 & 0.00488 & 0.00437 & 0.00519 & 0.00986 & $\mathbf{0 . 0 0 4 3 7}(2)$ \\
\hline Trial 3 & 0.00423 & 0.00632 & 0.00628 & 0.00724 & $0.00423(1)$ \\
\hline Trial 4 & 0.00678 & 0.00625 & 0.00612 & 0.00711 & $0.00612(3)$ \\
\hline Trial 5 & 0.00663 & 0.00606 & 0.00528 & 0.00612 & $0.00528(3)$ \\
\hline Average & 0.00511 & 0.00504 & 0.00649 & 0.00761 & 0.00463 \\
\hline
\end{tabular}

As it is illustrated in Table 3.1, the proposed NoSEE method results in obtaining the lowest value of RMSE in every case. Also, the average RMSE over 75 trials is displayed in the last row of every artifact-removal method, which shows a more accurate performance of NoSEE compares to manually fixing the number of components.

Table 3.2: The accuracy in \% describing the estimate of number of components (NoC) over 75 trials.

\begin{tabular}{|c||c||c||c|}
\hline & SSP & runICA & JADE \\
\hline \hline Proposed Method & $85.3 \%$ & $98.7 \%$ & $98.7 \%$ \\
\hline Manual Fixed NoC & $33.3 \%$ & $33.3 \%$ & $33.3 \%$ \\
\hline
\end{tabular}


Table 3.2 illustrates the high accuracy of the proposed method in all subspace-based algorithms. The row Manual Fixed NoC represents the accuracy of the method when NoC is fixed to be either1, 2, or 3. It can be seen that the accuracy in SSP is lower compared to other methods, which is predictable as SSP is more dependant on the number of blinks and the length of the data.

\subsubsection{Real MEG Result and Discussion of Proposed pre-processing Method}

Performing the proposed method on the real MEG data, provided us with a value of $m=2$ as the estimate for the number of components in blinking artifact. Therefore, our objective in this section is to analyze and validate this observation using the standard methods performed for manual selection of the number of components. Since the proposed method selected $m=2$, the first 4 components will be analyzed validate and exam the suggested NoC. One common way to evaluate the components and identify the components responsible for blinking is to analyze the topography of the component. Topography component illustrates the spatial distribution of each shown component on the head. This is shown in Figure 3.5. Four different topographies are responsible for the four first components where SPP1 is the first SSP projection component, SSP2 is showing the second component, and etc. The value shown in percentage illustrates normalized singular values (components). A higher percentage is an illustration of the greater influence of that component on overall blinking signals.



Figure 3.5: Topography of the first four components from concatenate MEG blinking artefacts

Topographical illustration of blinking activities often shown to beflat at every area of the topography except at frontal area. In the frontal area, abrupt polarity reversal is expected to be seen [84]. This is obvious and observable on the first component of the shown topography in Figure 3.5. The second component is most likely illustrating the Saccade, which is a type of eye movement, which is shown maximally onto anterior sites [84]. This is while no eye-related activities could be obtained from the third and fourth components by the topographical analysis. The second method for manually examining the components corresponding to blinking is comparing the times series data of the components of EOG data (if it is available). Figure 3.6 illustrates the first four components along with vertical and horizontal 
EOG labelled as VEOG and HEOG, respectively. By comparing the EOG activities with the first four
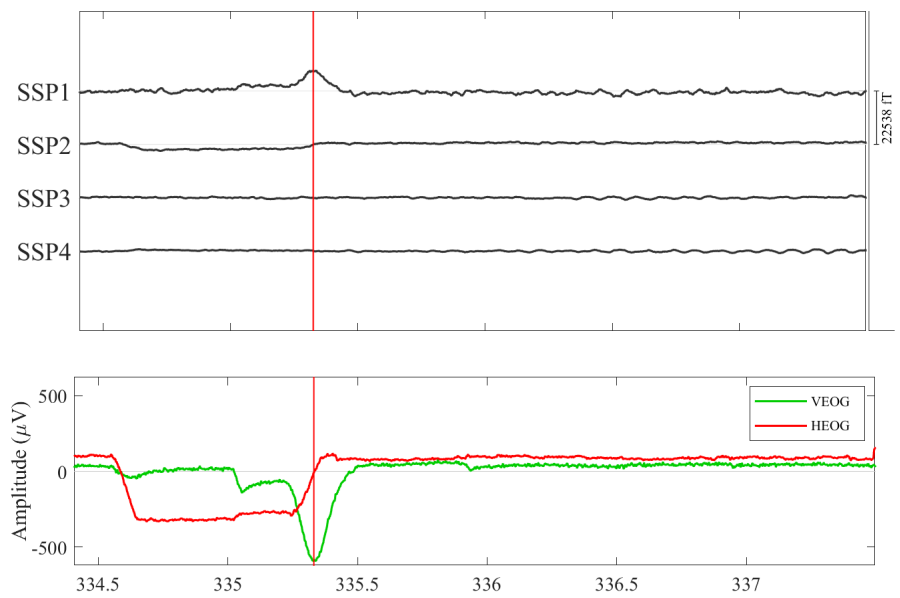

Figure 3.6: Comparison between EOG times series and the four first components

components, one can see that the first two components are capturing most of the EOG activities while the change in SP3 and SP4 are insignificant. One could see that the major peak shown in VEOG is mostly captured by the first component while step-like variation which is an illustration of Saccade components $[84,90]$ shown in the HEOG is obtained by the second component. Hence it is concluded that the manual inspection of the MEG data using SSP components analysis validates the number of blink components estimated by the proposed method. 


\section{Chapter 4}

\section{Automated Source Thresholding in Low Resolution Inverse Problems}

As explained earlier in the Section 2.7, among inverse problem formulations, there exists group of solutions which provide a reasonable estimate for the location of the source while being simple and computationally fast, such as the $L_{2}$-Regularization based category of solutions. However, these solutions tend to provide low resolution and blurred estimated. This chapter of the thesis is dedicated to describing the proposed post-processing algorithms in the form of automatic thresholding which would increase the resolution of the $L_{2}$-based inverse solutions. Various development in the initially proposed algorithm has occurred in the form of multiple publications, the proposed algorithms and their developments will be explained in chronological order.

\subsection{AESET in $L_{2}$-Regularization Inverse Problems}

In the context brain source localization, $L_{2}$-regularization based solutions are computationally fast and simple due to the fact that the $L_{2}$-norm has a closed form solution. Solutions with $L_{2}$-norm regularization term, are over smooth and lack the required accuracy needed for many applications. Therefore, postprocessing thresholding is usually implemented with these algorithms. However, the value of these thresholds is set manually, causing loss of relevant information, important sources or low accuracy in the estimate. Here a new thresholding algorithm that automatically chooses the optimum threshold in the sense of $L_{2}$-Norm denoising and based on Minimum Noiseless Description Length (MNDL) thresholding is introduced. The results show that the proposed thresholding overcomes the existing problem of manual thresholding and provides accurate results without missing any sources.

Consider (2.26) expression, which illustrates how source estimate is obtained at any given sample. The post-processing step of thresholding set a threshold on the result of $\hat{J}(n)$ amplitudes and indicate that any value less than the mentioned threshold will be replaced by zero through hard thresholding. The primary assumption of our proposed method for automating this thresholding is that the existing noise 
in the obtained $\hat{J}(n)$ have WGN characteristic. This is a reasonable assumption since many $L_{2}$-based inverse problems have pre-assumption that the noise has WGN [91], moreover, in many methods, the closed-form expression provided for each inverse problem solution is whitened [92, 93] before computation of $\hat{J}$. This allows us to write the obtained source estimate as follows:

$$
\hat{J}=J+\omega
$$

where $\omega$ has WGN distributed on the grid points with variance $\sigma_{\omega}$. Since the amount of activation of a source within the grid is shown with the amplitude of the source for that grid, i.e., higher amplitude means it is more likely that the grid point contains the source, simple thresholding can denoise the source to some extent. Therefore, setting a threshold on the result of the source estimate observed from $L_{2}$-Regularization methods is a common practice. Thresholding is done to detect significant activities within the brain and to be able to localize the active source with higher resolution.

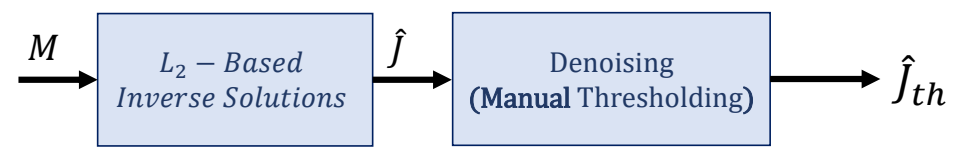

Figure 4.1: Existing $L_{2}$-Regularization post-processing (Thresholding)

As shown in Figure 4.1, thresholding is currently done manually. This makes the performance of the methods rather subjective. Since in the case of real EEG source localization, the ground truth is unknown, manual thresholding is shown to become troublesome, unreliable and can induce the risk of losing valuable information. In this work, a new rigorous automated thresholding to eliminate manual thresholding is introduced.

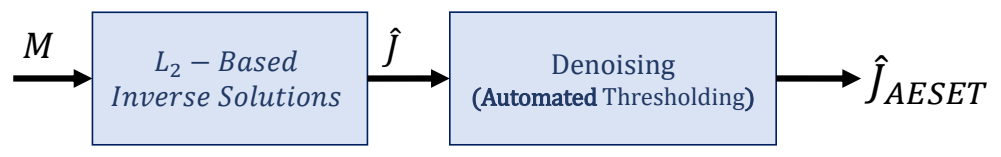

Figure 4.2: Proposed automated post processing (Automated EEG Source Error Thresholding (AESET) threholding)

\subsection{Automated EEG Source Error Thresholding (AESET)}

Hard thresholding of values of $\hat{J}$ with a threshold value $m(\tau)$ is equivalent to sorting these values and choosing the first $m$ values $\left(J_{\tau} \rightarrow J_{m(\tau)}\right)$. Therefore there is a map from threshold value $\tau$ to a value of $m$ between zero and $N_{s}$ (length of $J$ ). Consequently, the choice of optimum threshold is equivalent to the choice of the optimum subspace selection and the optimum value of $m$. For each value of the 
threshold, and therefore $m$, the following error is available:

$$
\text { Available Source Error: } X_{m}=\frac{1}{N_{s}}\left\|\hat{J}-\hat{J}_{m(\tau)}\right\|_{2}^{2}
$$

By Maximum Likelihood approach, it is known that as the value of $m$ is increased( lowering the threshold) this error monotonically approaches zero. The optimum value of $m$ such that it provides the minimum description length (DL) of the data is intended to be find. Based on 4.1 the PDF of the source estimate is:

$$
f(\hat{J} ; J)=\frac{1}{\left(\sqrt{2 \pi \sigma^{2}}\right)^{N_{s}}} e^{\frac{-\|\hat{J}-J\|_{2}^{2}}{2 \sigma}}
$$

The objective is to chose value of $\mathrm{m}$ such that the noiseless description length is minimized (MNDL) $[94,95]$ :

$$
m_{A E S E T}=\arg \min _{m(\tau)} \mathrm{DL}\left(J ; \hat{J}_{m(\tau)}\right)
$$

where

$$
\mathrm{DL}\left(J ; \hat{J}_{m(\tau)}\right)=-\log _{2}\left(f\left(\hat{J}_{m(\tau)} ; J\right)\right)
$$

Based equations (4.3) and (4.5), minimizing the description length is equivalent to minimizing following unavailable error:

$$
\text { Desired Source Error: } Z_{m(\tau)}=\frac{1}{N_{s}}\left\|J-\hat{J}_{m(\tau)}\right\|_{2}^{2}
$$

While this error is not available, it is shown in $[96,75]$ that using the available source error the mean and variance of $Z_{m(\tau)}$ can be estimated with confidence and validation probabilities $P(Q(\alpha))^{1}$ an $P(Q(\beta))$, where $\alpha$ and $\beta$ are 4 for the probabilities to be 0.99 . Consequently, the probabilistic upper bound of $Z_{m(\tau)}$ is in the form of:

$$
\bar{Z}_{m(\tau)}=E\left(Z_{m(\tau)}\right)+\beta \sqrt{\operatorname{var}\left(Z_{m(\tau)}\right)}
$$

In this case the optimum value of $m$ in (4.4) is estimated based on the probabilistic worst-case scenario as following:

$$
\hat{m}_{A E S E T}=\arg \min _{m(\tau)} \bar{Z}_{m(\tau)}
$$

The pseudo-code for AESET is provided in Algorithm 1.

$$
{ }^{1} Q(\alpha)=\int_{-\alpha}^{\alpha}\left(\frac{1}{\sqrt{2 \pi}}\right) e^{\frac{-x^{2}}{2}} d x
$$




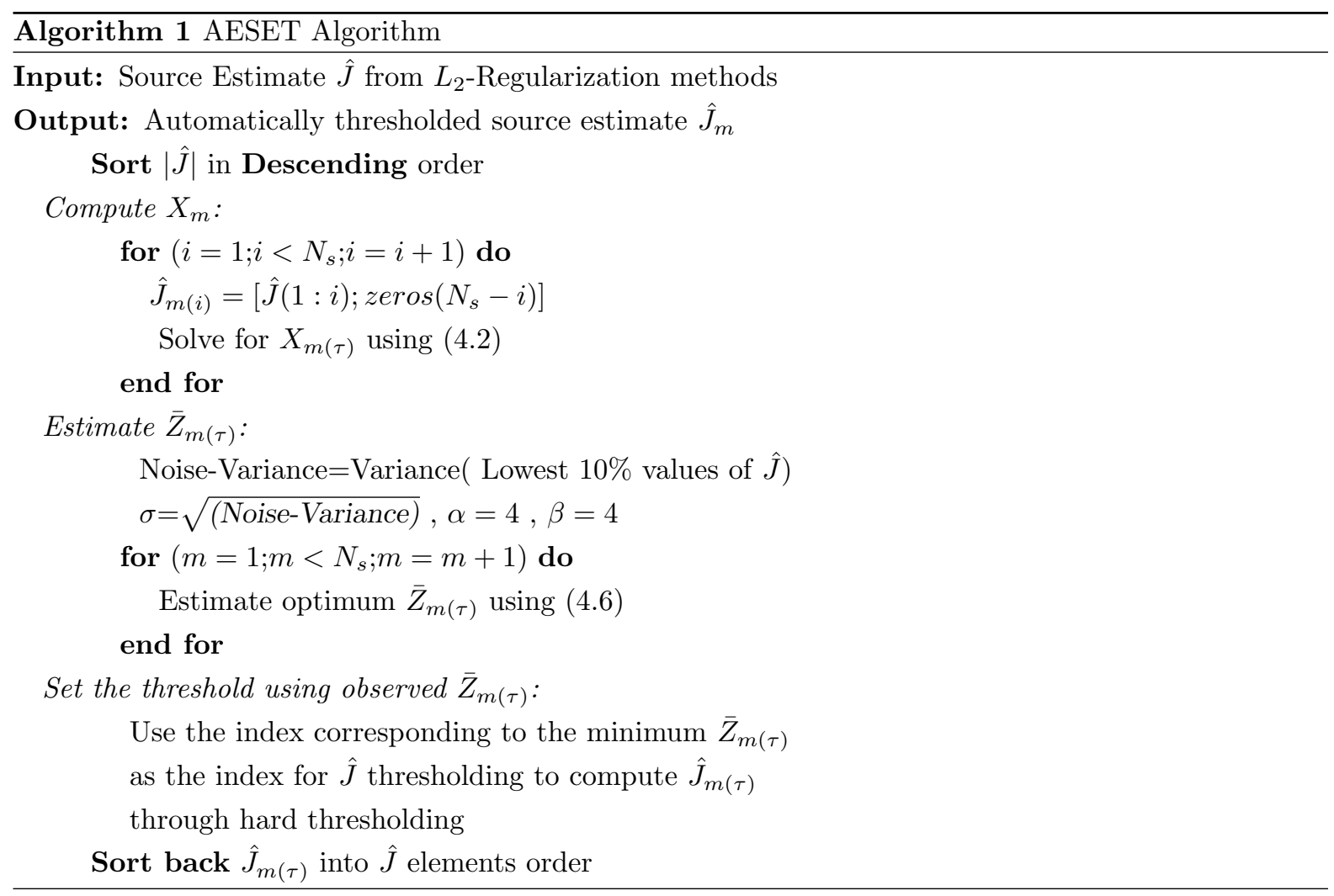

\subsubsection{Simulation of AESET}

To evaluate the performance of AESET thresholding method, multiple simulations were performed using synthetic data. The active sources were constructed for one, two and three patches of the source, where each source within the patch is restricted to be perpendicular to the cortical surface [97]. For each case, the EEG simulation was observed by applying the lead-flied matrix to the constructed sources $(H J)$, while noise level varied in each scenario to achieve the Signal-to-Noise Ratio (SNR) of 5dB, $10 \mathrm{~dB}$ and $15 \mathrm{~dB}$. SNR is defined as the logarithmic ratio between the power of the signal and power of the noise [97]. Evaluation of the algorithm performance was done with Brainstorm [28], which is freely available for download online under GNU general public license. ICBM152 anatomy, distributed by the Montreal Neurological Institute (MNI) [98] was used as the default anatomy with 15002 as its number of vertices for the cortex surface. In the simulation, 41 electrode channels were used. Three-layered spherical head model was used as the forward model for both simulation and the inverse solution [99]. The proposed method is examined on the most popular linear inverse model that use $L_{2}$-Regularization source estimation methods, WMNE, DSPM, and SLORETA. The manual thresholding values of 3\%, $10 \%$ and $70 \%$ were used for the comparison as suggested in [100], [101], and [102] respectively. 


\subsubsection{Evaluation Criteria for AESET method}

Two types of error measurement are relevant in this scenario, and are used to evaluate the performance of the thresholding method:

1- Mean Square Error (MSE)[103], which is defined as the $L_{2}$-norm difference between reconstructed current and the ground truth.

$$
M S E=\frac{\|\hat{\nu}-\bar{\nu}\|_{2}}{\|\bar{\nu}\|_{2}}
$$

where $\bar{\nu}$ is the ground truth for the source observed from $J$ and $\hat{\nu}$ is the estimated source with different values of thresholding. In the source imaging problem, lower MSE is desired as long as the sources are not missed.

2- Percentage of Undetected Source number (PUS) [104], which is defined as the real source whose location to its estimate is greater than 0.6 times the unit distance of the source. Here, the patches of the sources and the average radius of their area as the unit distance is used.

$$
P U S=\frac{N_{u n}}{N_{\text {real }}}
$$

where $N_{u n}$ is the number of undetected sources and $N_{\text {real }}$ illustrate the total number of sources.

\subsubsection{Simulation Results of AESET}

Figure 4.3 illustrates the performance of the three methods with different thresholds at $5 \mathrm{~dB}$ SNR. As can be seen from the plots, very high thresholding such as the threshold of $70 \%$ would lead to losing important information in the estimate. Additionally, lower thresholds cause the solution not to be accurate enough. The proposed method AESET is adaptive in the sense that it uses statistical information about the source estimate to perform thresholding. As can be observed from the figure, in most of the cases AESET method outperforms the manual thresholds in the sense of providing a more accurate solution without losing important information. 


\section{TRUE SOURCE}

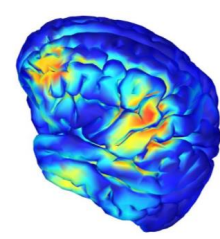

(a) SLORET A $\%$

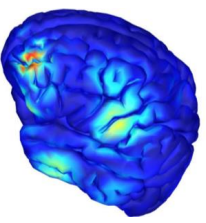

(e) $W M N E_{0} \%$

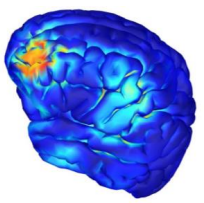

(j)dSPM $M_{0} \%$

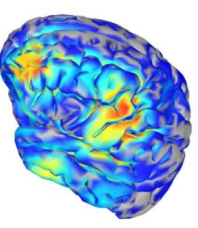

(b) SLORET A $_{3} \%$

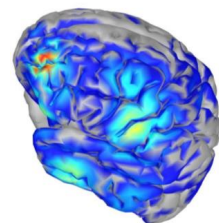

(f) $W M N E_{3 \%}$

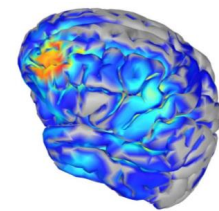

(k)dSPM $M_{3 \%}$
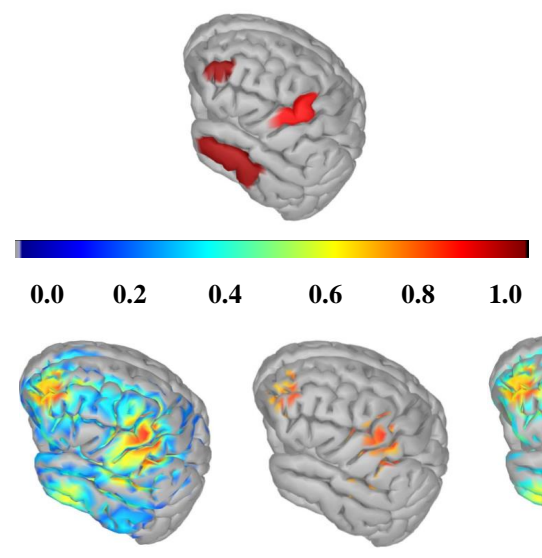

(c) SLORET A $_{10 \%}$
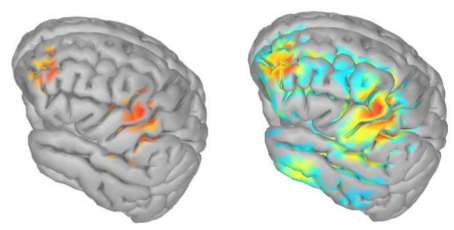

(d) SLORETA $_{70 \%}$

(d) SLORET A $A_{\text {AESET }}$



(g) $W M N E_{10} \%$

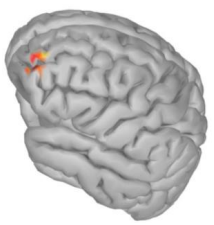

(h) $W M N E_{70 \%}$

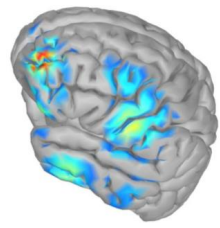

(i) WMNE $E_{A E S E T}$

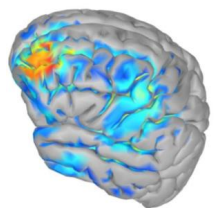

(l) $d S P M_{10 \%}$

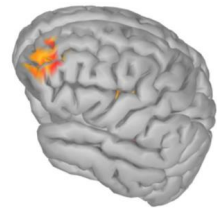

(m) $\boldsymbol{d S P M}_{\mathbf{7 0} \%}$

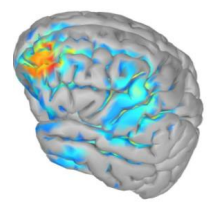

(n)dSPM $M_{A E S E T}$

Figure 4.3: Three sources estimation evaluation for SNR of 5dB. The subscript under each method illustrate the amount of thresholding in percent.

It should be mentioned that visual inspection of the result from the figure is not enough as there may be underlying activities in the depth of the cortex that are not see-able by the figure. Table 4.1 provides quantitative information with regards to the performance of the method, for three SNRs and for three cases with one, two, or three sources. The information provided in the table is the result of averaging twenty trails MSE and PUS, wherein each simulation noise, amplitude and time delay of sources, size and location of the patches and the number of sources within each patch varied randomly. Note that in PUS the objective is to see if any of the sources will be lost in the process of thresholding by imposing different scenarios, and here minimum MSE is desired in source imaging methods as long as the thresholding method does not miss the sources (PUS=0). As the table illustrates, only for low SNRs and one source $70 \%$ thresholding outperforms AESET and this is due to the error in noise variance estimation of the approach. 
Table 4.1: MSE (PUS), Averaged over 20 trials for three cases (One Source, Two Sources and Three sources

\begin{tabular}{cccccc}
\hline Methods & One Source & Two Source & Three Source & Three Source & Three Source \\
\hline & $5 \mathrm{~dB}$ & $5 \mathrm{~dB}$ & $5 \mathrm{~dB}$ & $10 \mathrm{~dB}$ & $15 \mathrm{~dB}$ \\
\hline sLORETA $_{0 \%}$ & $27(0 \%)$ & $25(0 \%)$ & $23(0 \%)$ & $22(0 \%)$ & $22(0 \%)$ \\
sLORETA $_{3 \%}$ & $26(0 \%)$ & $25(0 \%)$ & $23(0 \%)$ & $22(0 \%)$ & $22(0 \%)$ \\
sLORETA $_{10 \%}$ & $24(0 \%)$ & $23(0 \%)$ & $22(0 \%)$ & $19(0 \%)$ & $21(0 \%)$ \\
SLORETA $_{70 \%}$ & $\mathbf{6}(0 \%)$ & $7(30 \%)$ & $7(32 \%)$ & $6(41 \%)$ & $5(47 \%)$ \\
sLORETA $_{A E S E T}$ & $20(0 \%)$ & $\mathbf{1 8}(\mathbf{0} \%)$ & $\mathbf{1 7}(0 \%)$ & $\mathbf{1 9}(0 \%)$ & $\mathbf{1 9}(0 \%)$ \\
WMNE $_{0 \%}$ & $9(0 \%)$ & $8(0 \%)$ & $6(0 \%)$ & $7(0 \%)$ & $6(0 \%)$ \\
WMNE $_{3 \%}$ & $8(0 \%)$ & $8(0 \%)$ & $6(0 \%)$ & $7(0 \%)$ & $6(0 \%)$ \\
$\mathrm{WMNE}_{10 \%}$ & $6(0 \%)$ & $\mathbf{6}(0 \%)$ & $\mathbf{4}(0 \%)$ & $\mathbf{4}(0 \%)$ & $6(0 \%)$ \\
$\mathrm{WMNE}_{70 \%}$ & $\mathbf{2 ( 0 \% )}$ & $2(32 \%)$ & $1(64 \%)$ & $1(62 \%)$ & $1(58 \%)$ \\
$\mathrm{WMNE}_{A E S E T}$ & $6(0 \%)$ & $\mathbf{6}(0 \%)$ & $\mathbf{4}(0 \%)$ & $\mathbf{4}(0 \%)$ & $\mathbf{4}(0 \%)$ \\
$\mathrm{dSPM}_{0 \%}$ & $24(0 \%)$ & $25(0 \%)$ & $27(0 \%)$ & $27(0 \%)$ & $27(0 \%)$ \\
$\mathrm{dSPM}_{3 \%}$ & $25(0 \%)$ & $25(0 \%)$ & $26(0 \%)$ & $26(0 \%)$ & $27(0 \%)$ \\
$\mathrm{dSPM}_{10 \%}$ & $22(0 \%)$ & $24(0 \%)$ & $25(0 \%)$ & $23(0 \%)$ & $24(0 \%)$ \\
$\mathrm{dSPM}_{70 \%}$ & $\mathbf{6}(0 \%)$ & $7(37 \%)$ & $6(37 \%)$ & $9(32 \%)$ & $8(33 \%)$ \\
$\mathrm{dSPM}_{A E S E T}$ & $19(0 \%)$ & $\mathbf{2 3}(0 \%)$ & $\mathbf{2 0}(0 \%)$ & $\mathbf{2 1}(0 \%)$ & $\mathbf{1 9}(0 \%)$ \\
\hline
\end{tabular}

As shown in the table, the over-smoothness of the $L_{2}$-regularization often leads to observing no undetected sources, unless the employed thresholding is more than required and cause loss of information as the thresholding of $70 \%$ illustrate. Note that although the MSE of $70 \%$ thresholding is smaller than all the other methods, it misses the sources once there is more than one source.

\subsubsection{Analysis of the Obtained Results for Further Improvements on AESET method}

As it was mentioned earlier, the proposed method here considers the lowest $10 \%$ values of the $\hat{J}$ as the representation of noise and its variance as a noise variance estimate. The suggested method for noise variance estimate is uncertain, and the suggested $10 \%$ tail, is not optimum under all conditions. To further improve the method, as it will be described in the next section, a new data-driven noise variance estimate is proposed. The proposed method is performed and evaluated solely on SLORETA, and this is because SLORETA is the most popular method and AESET method have illustrated acceptable performance on this method. 


\subsection{HR-sLORETA in EEG Source Imaging}

The proposed approach here describes improvements that were done on the automated threshold method explained above. Due to the popularity of SLORETA compare to all other mentioned methods, and efficient performance of AESET on this method, further improvements were performed only on SLORETA. The proposed method is introduced as Higher Resolution sLORETA (HR-sLORETA). While the method follows the same procedure and problem formulation, a new noise variance estimation is introduced, which is described in the following section.

\subsubsection{Noise Variance Estimation Using Kurtosis Analysis}

It is shown in [75] that the desired source error in (4.6) is a Gaussian distribution with the following variance:

$$
\operatorname{var}\left(Z_{m}\right)=\frac{2 m}{N_{s}^{2}}\left(\sigma_{\omega}^{2}\right)^{2}
$$

As the equation above illustrates, to find a solution for (4.11), an estimate of the noise variance $\sigma_{\omega}^{2}$ is required. Sorting the source estimate $\hat{J}$ in descending order and choosing the last $N s-i$ values as the estimate of noise in $\hat{J}$ is an acceptable assumption as the lowest values in the source estimate represent the noise. Additionally, the standardization that is done in SLORETA suppress noise within the estimate which further supports the assumption. Hence the primary question is the optimum value of $i$ that is representing the noise estimate within the source estimate. Estimating this value by an iterative process within a range of values between $i_{\max }$ and $i_{\min }$ is proposed. For each value of $i$, the estimated noise variance is

$$
\hat{\sigma}_{\omega}^{2}(i)=\frac{1}{N_{s}-1} \sum_{p=i}^{N_{s}}\left\|\hat{J}_{p}-\mu_{i}\right\|^{2}
$$

where:

$$
\mu_{i}=\frac{1}{N} \sum_{p=i}^{N_{s}} \hat{J}_{p}
$$

Therefore, for each value of $i$ optimum denoised value that is a function of $i$ is defined:

$$
i \rightarrow \hat{\sigma}_{\omega}^{2}(i) \rightarrow \hat{J}_{m^{*}}(i)
$$

Once $\hat{J}_{m^{*}}(i)$ corresponding to $\hat{\sigma}_{m}^{2}(i)$ is obtained, an estimate of subspace noise can be calculated:

$$
\hat{\omega}(i)=\hat{J}-\hat{J}_{m^{*}}(i)
$$

The result of 4.15 illustrates the difference between the SLORETA source estimate and HR-sLORETA, which is the noise within the SLORETA source estimate. Inspired by ICA denoising method described in Section 2.4.3, the optimum value for $i$ is chosen by an error criteria constructed based on the the kurtosis of the subspace noise. Kurtosis is standardized fourth moment about the mean and is formulated for 
the estimated subspace noise as follows [105]:

$$
\kappa_{\hat{\omega}(i)}=\frac{E\left(\hat{\omega}(i)-\mu_{\hat{\omega}(i)}\right)^{4}}{\left(E\left(\hat{\omega}(i)-\mu_{\hat{\omega}(i)}\right)^{2}\right)^{2}}=\frac{\mu_{4}(i)}{\sigma_{\hat{\omega}(i)}^{4}}
$$

where $\mu_{\hat{\omega}(i)}$ and $\sigma_{\hat{\omega}(i)}$ are mean and standard deviation of the subspace noise estimate respectively. As the primary assumption is that the noise has a Gaussian distribution, this value has to be compared with the optimum value for the normal distribution that is 3 [105]. Using this statistical knowledge about the characteristic of the noise to define an error, the Kurtosis Error can be defined as:

$$
\epsilon_{\kappa}(i)=\| 3-\left|\kappa_{\hat{\omega}(i)}\right|||^{2}
$$

The optimum value for the $i$ is the value that minimizes the Kurtosis Error and equivalently represents the most consistency between the prior assumption on the additive noise and resulted residuals of the algorithm:

$$
i^{*}=\arg \min _{i} \epsilon_{k}(i)
$$

which lead to the optimum value of the denoised source estimate $\hat{J}_{m^{*}}\left(i^{*}\right)$.

\subsubsection{HR-sLORETA Simulation}

EEG source imaging of SLORETA is performed using Brainstorm [28], a MATLAB toolbox freely available for download online under GNU general public license. The anatomy of the head is constructed using MNI Colin 27 [106], and three-layered spherical head model [107] is used for both forward model and inverse model. The number of potential sources (grid on the cortex surface) is set to 15002 with 65 electrode measures as the number of sensors. The sources are defined as patches of neurons, where the orientation of the neurons are restricted to be perpendicular to the cortical surface. Moreover, the sources are positioned randomly on the surface of the cortex (grids). For each patch, a synthetic source is generated, which represent the summation of all existing neurons in the patch. The variation in source patch signals is with the introduction of time-shift and amplitude change to mimic the propagation of source activation across the brain. Additionally, a normally distributed noise with various amplitude is added in each simulation, which corresponds to different SNR values. EEG simulation is constructed by multiplying the synthetic sources by the gain matrix. While the total number for potential sources $\left(N_{s}\right)$ is set to 15,000 , it is empirically observed that even in the higher number of sources such as the one shown in Figure 4.4, the number of active sources does not exceed $300-400$ points. Therefore the range of values for $i$ are set to $i_{\min }=500$ and $i_{\max }=2000$ which corresponds to that the last 14,500 to 13, 000 points respectively. The method is examined using one, two, three and four patches of sources with SNR values of $5 \mathrm{~dB}, 10 \mathrm{~dB}$, and $15 \mathrm{~dB}$. The result shown here is the average of 10 trials. The method is compared with manual thresholds of $3 \%$ [108], 10\% [109] and 70\% [110] and the automatic threshold using Otsu [111] as suggested in [112]. ( Note that in addition to manual thresholding, a gray-level thresholding algorithm introduced by Otsu [111] is also suggested in [112], as a method of automatic 
thresholding).

\subsubsection{Evaluation Criteria for HR-sLORETA}

One of the important characteristics in improving the solution of SLORETA is to introduce no additional False Negative (FN) compared to the original SLORETA. This means that the method does not cause loss of any sources. This is evaluated by PUS [113], which was described previously. The result of PUS criteria is shown by percentage (\%), and any value other than 0 suggests that the method is missing a source; and since missing the source is in contrast with the motivation of this paper, any method that illustrates PUS $>0 \%$ will be eliminated. The second criteria used in this paper is Spatial Dispersion (SD) [114]. Spatial Dispersion measures the resolutions of the source estimate in the unit of $m m$. The lower the value, the higher is the resolution in the source imaging and it is defined as follow:

$$
S D_{r}=\sqrt{\frac{\sum_{k=1}^{N}\left(d_{k l} \cdot\left\|\hat{J}_{k l}\right\|\right)^{2}}{\sum_{k=1}^{N}\left\|\hat{J}_{k l}\right\|^{2}}}, l=1, \ldots, N_{s}
$$

where $d_{k l}$ is defined as the euclidean distance between the true source located at grid point $r$ and its closest source estimate at the location $k$. 


\subsubsection{Simulation Result and Discussion of HR-sLORETA}

The method is evaluated under four different scenarios where the number of sources increases, and for each scenario, SNR values of $5 \mathrm{~dB}, 10 \mathrm{~dB}$ and $15 \mathrm{~dB}$ were examined. Figure 4.4 illustrates one trail of four sources and performance of each method. As it is shown in Figure 4.4, HR-sLORETA is providing the highest resolution without loss of any source patch. One must note that visual observation is not enough since deeper regions of the cerebral cortex is not visible. Table 4.2 provides the result and performance of the method averaged over ten trails.

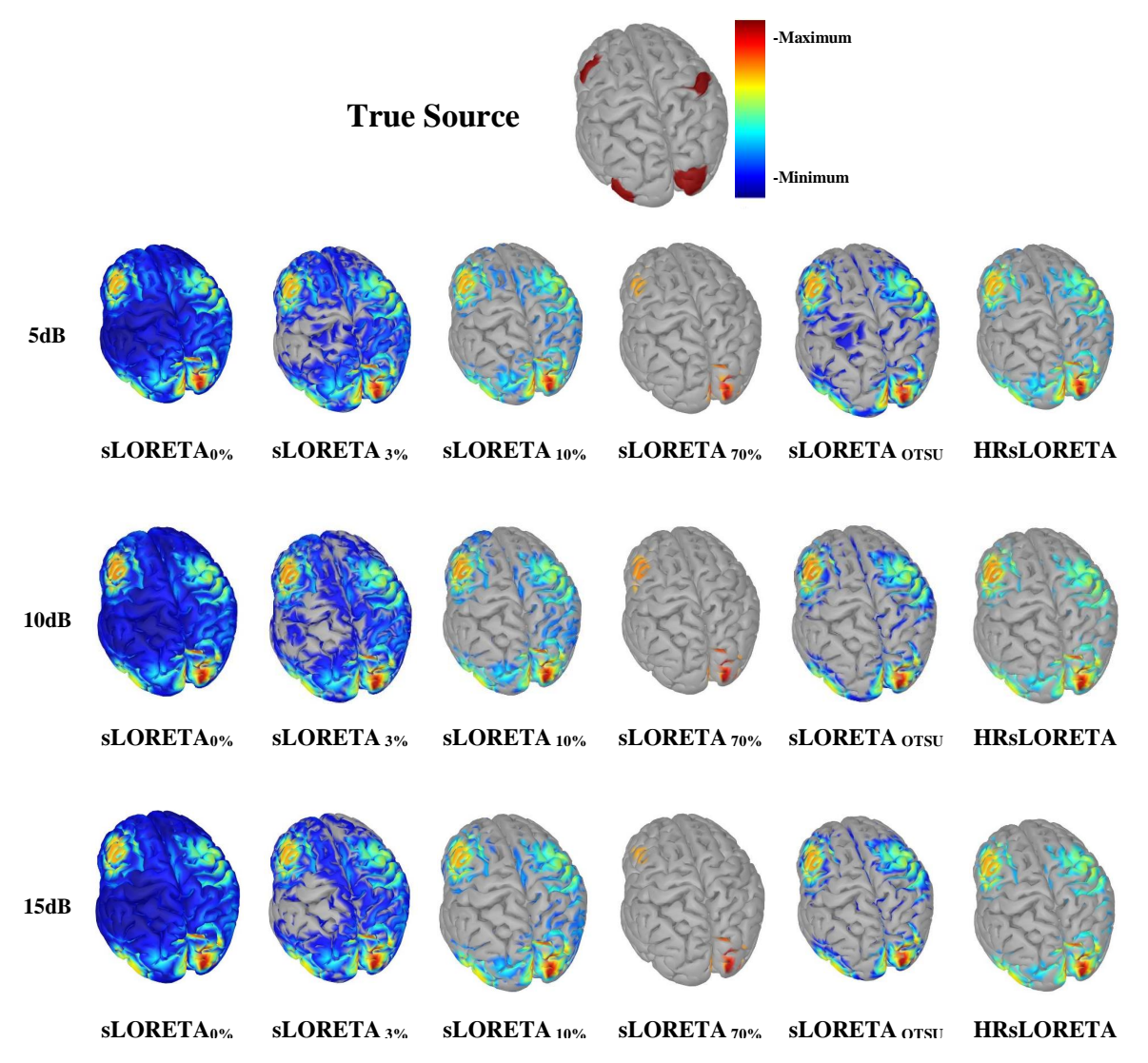

Figure 4.4: Illustration of SLORETA with various thresholds SLORETA for the case of 4 sources.

As it is shown in Figure 4.4, SLORETA $_{70 \%}$ which corresponds to the solution of SLORETA with manual thresholding of $70 \%$ illustrates losing two of the four source patches. Using the evaluation method PUS, the performance of the method in terms of having any False Negative (FN) is evaluated. The observed result shows that the only method that has non-zero PUS, PUS $>0 \%$ is SLORETA $_{70 \%}$. The value of PUS was zero for all other methods suggesting no information was lost in the process. The threshold SLORETA $_{70 \%}$, shows PUS $=50 \%$ for two sources and PUS of $30 \%$ to $60 \%$ for three and four sources on average over ten trails. Therefore for further evaluations, SLORETA $_{70 \%}$ method is eliminated in the following analysis. Using Spatial Dispersion which evaluates the method's resolution, HR-sLORETA 
along with the mentioned competitors are evaluated. The results are illustrated in Table 4.2. As the table shows, at the lower values of source patches, Otsu method is comparable with HR-sLORETA. Note that Otsu is a faster algorithm and have the computational time of under 1 second while HR-sLORETA requires a 3-4 seconds to run. However, as the number of sources increases, the performance of the Otsu drastically degrades. In fact, for any of the trials where the number of sources is greater than two, the Otsu method performs very poorly. This is an expected issue that has been discussed in the application of the Otsu thresholding method for gray-level images (As the intensity of background activity increase, the performance of the gray-level threshold decrease). HR-sLORETA shows robust performance in the presence of a different number of sources. This is quite beneficial in applications where source imaging is used, and the number of sources is high than three as for the examples in [115, 116]. Table 4.2: SD in mm Averaged over 10 trials

\begin{tabular}{|c|c|c|c|c|}
\hline Method & One Source & Two Source & Three Source & Four Source \\
\hline \multicolumn{5}{|l|}{$5 \mathrm{~dB}$} \\
\hline sLORETA $_{0 \%}$ & 818.61 & 140.06 & 36787 & 15170.91 \\
\hline sLORETA $_{3 \%}$ & 200.56 & 72.72 & $180 . .33$ & 294.18 \\
\hline sLOTERA $_{10 \%}$ & 27.92 & 22.21 & 66.61 & 53.35 \\
\hline sLORETA $_{O t s u}$ & 14.45 & 6.88 & 142.09 & 119.85 \\
\hline HR-sLORETA & 27.42 & 16.31 & 23.75 & 31.21 \\
\hline \multicolumn{5}{|l|}{$10 \mathrm{~dB}$} \\
\hline sLORETA $_{0 \%}$ & 2173.74 & 131.57 & 246.21 & 3463.20 \\
\hline sLORETA $_{3 \%}$ & 175.29 & 60.67 & 185.94 & 289.29 \\
\hline sLOTERA $_{10 \%}$ & 27.07 & 23.01 & 68.51 & 55.8 \\
\hline sLORETA $_{O t s u}$ & 13.12 & 6.39 & 55.82 & 79.99 \\
\hline HR-sLORETA & 19.9 & 13.19 & 19.5 & 30.24 \\
\hline \multicolumn{5}{|l|}{$15 \mathrm{~dB}$} \\
\hline sLORETA $_{0 \%}$ & 21273.05 & 123.78 & 8919.77 & 7194.19 \\
\hline sLORETA $_{3 \%}$ & 182.32 & 53.1 & 5272.19 & 294.87 \\
\hline sLORETA $_{10 \%}$ & 26.85 & 22.56 & 25.94 & 55.39 \\
\hline sLORETA $_{O t s u}$ & 13.15 & 6.96 & 126.70 & 76.94 \\
\hline HR-sLORETA & 17.81 & 11.51 & 19.17 & 29.21 \\
\hline
\end{tabular}

The performance of the methods can be further investigated by analyzing the standard deviation of the SD errors as the number of sources increases. Table 4.2 illustrates the standard deviation of Spatial Dispersion Error for Otsu and HR-sLORETA methods as they are the most competitive methods in the above experiments. As the table shows, standard deviation of SD in Otsu thresholding is comparable with the value of its SD, especially in low SNRs, whereas the SD standard deviation of HR-sLORETA is smaller than the SD itself for the proposed method. This confirms the robustness of HR-sLORETA 
compares to the Otsu approach.

Table 4.3: Standard Deviation of SD Error for Otsu and HR-sLORETA.One S, Two S, Three S, and Four $S$ illustrate using one source to four sources in simulation respectively.

\begin{tabular}{|c|c|c|c|c|}
\hline Method & One $\mathrm{S}$ & Two S & Three S & Four S \\
\hline \multicolumn{5}{|l|}{$5 \mathrm{~dB}$} \\
\hline sLORETA $_{O t s u}$ & 2.43 & 0.81 & 107.34 & 48.98 \\
\hline HR-sLORETA & 4.06 & 2.29 & 5.36 & 9.96 \\
\hline \multicolumn{5}{|l|}{$10 \mathrm{~dB}$} \\
\hline sLORETA $_{O t s u}$ & 1.31 & 0.26 & 10.51 & 12.47 \\
\hline HR-sLORETA & 2.18 & 1.11 & 2.30 & 3.05 \\
\hline \multicolumn{5}{|l|}{$15 \mathrm{~dB}$} \\
\hline sLORETA $_{O t s u}$ & 0.85 & 0.24 & 11.26 & 8.40 \\
\hline HR-sLORETA & 1.78 & 0.97 & 2.52 & 3.57 \\
\hline
\end{tabular}

\subsection{Improved HR-sLORETA}

The proposed method (HR-sLORETA) provides acceptable and stable performance. Yet, the method could be further improved because of the following reasons:

- Otsu method still illustrates a better performance for a single source case.

- Although the noise variance estimation through kurtosis analysis introduced in Section 4.3.1, provides an acceptable estimate, the obtained estimate is not optimum, and further improvements could be made.

In Section 4.4.1, a new noise variance estimate is introduced and explained.

\subsubsection{Noise Variance Estimation Using Kurtosis and Entropy Analysis}

The method introduced in Section 4.3.1 for obtaining a noise variance estimation is an iterative process, which evaluates various noise variance representations and selects the one with the minimum kurtosis error defined in (4.17). In each iteration, the noise variance estimation is obtained from the tail of the ordered data. In this case, the variable that changes with each iteration is the selected length of the tail; therefore the desired length of the tail that represents the best noise variance estimation is the objective of the defined loop. The critical analysis of this method raised two important flaws, which are described here:

1. Using the tail of the data itself is not the appropriate subject, because: 
- The tail-range selection method enforces the noise variance estimation to be dependant on the amplitude of the tail of the data. In the cases where the difference between the maximum and the minimum amplitude of the data is extreme, noise variance estimation from the data-tail may be miss-leading. This means that in some cases, the true noise variance estimate may not be in the subspace of the selected range.

- By using the tail range of the data, it is difficult to evaluate and analyze the range of the noise variance that has been tested, unless for every iteration, explicitly noise variance estimate is computed.

2. Minimizing the kurtosis error in (4.17) ensures that the selected noise variance estimate leads to noise with normal distribution after the process of thresholding. However, this method does not guarantee that the selected noise variance estimate is optimum.

To compensate for the mentioned flaws in the noise variance estimate, a new version noise variance estimate is introduced, which have the following characteristics:

1. Instead of using the tail of the data for the noise variance estimation, a specific range is given for the noise variance estimate, that will be evaluated in the iterative process (i.e. examine values for noise variance set between 0.1 to 4 with a defined increment).

2. Another well-defined characteristics of the normal distribution is having maximum entropy [117], [33] compare to all other distributions. This means that there is a trade-off between minimizing the kurtosis and maximizing the entropy. Therefore, selecting a noise variance estimation that could maximize the entropy of the resultant noise in addition to the previously mentioned kurtosis error could be used as an error function.

Considering that now the value of $i$ corresponds to iteration process among various noise variance estimates (i.e. $\sigma_{\omega}^{2}(i)$ ), the following cost function is defined to obtain optimum $i$ and therefore optimum noise variance estimate:

$$
i^{*}=\arg \min _{i}\left(\epsilon_{k}(i)-\epsilon_{E n t}(i)\right)^{2}
$$

where $\epsilon_{k}(i)$ is given in (4.17) and $\epsilon_{E n t}(i)$ is :

$$
\epsilon_{E n t}(i)=-\sum_{l=1}^{l=L}\left(p \log _{2}(p)\right)
$$

where $L$ is the length of the data and $p$ is the probability of the data obtained from the histogram [118]. Similar to the previous work, obtaining the optimum $i^{*}$ leads to denoised estimate $\hat{J}_{m^{*}}\left(i^{*}\right)$. 


\subsubsection{Improved HR-sLORETA Simulation and Evaluation}

The experimental setting for the simulation and evaluation of Improved HR-sLORETA is similar to the one explained in Section 4.3.2 and Section 4.3.3 respectively. Note that in this case, the range of $i$ defines the range for noise variance estimate. For this simulation $i_{\min }=0.1$ and $i_{\max }=4$ with the increments of 0.1. The method is examined by using one, two, three patches of sources with SNR value of $5 \mathrm{~dB}$ to illustrate the worst-case scenario. The method is compared with previously introduced HR-sLORETA as well as the Otsu automatic thresholding method [111]. Similar to the previous experiment in Section 4.3.3, qualitative analysis and SD are used to evaluate the method.

\subsubsection{Simulation Result and Discussion of Improved HR-sLORETA}

The qualitative analysis of the method is illustrated in Figure 4.5. The figure illustrates the performance

of the Otsu method, HR-sLORETA, and Improved HR-sLORETA methods on one, two and three sources for the SNR of $5 \mathrm{~dB}$. 


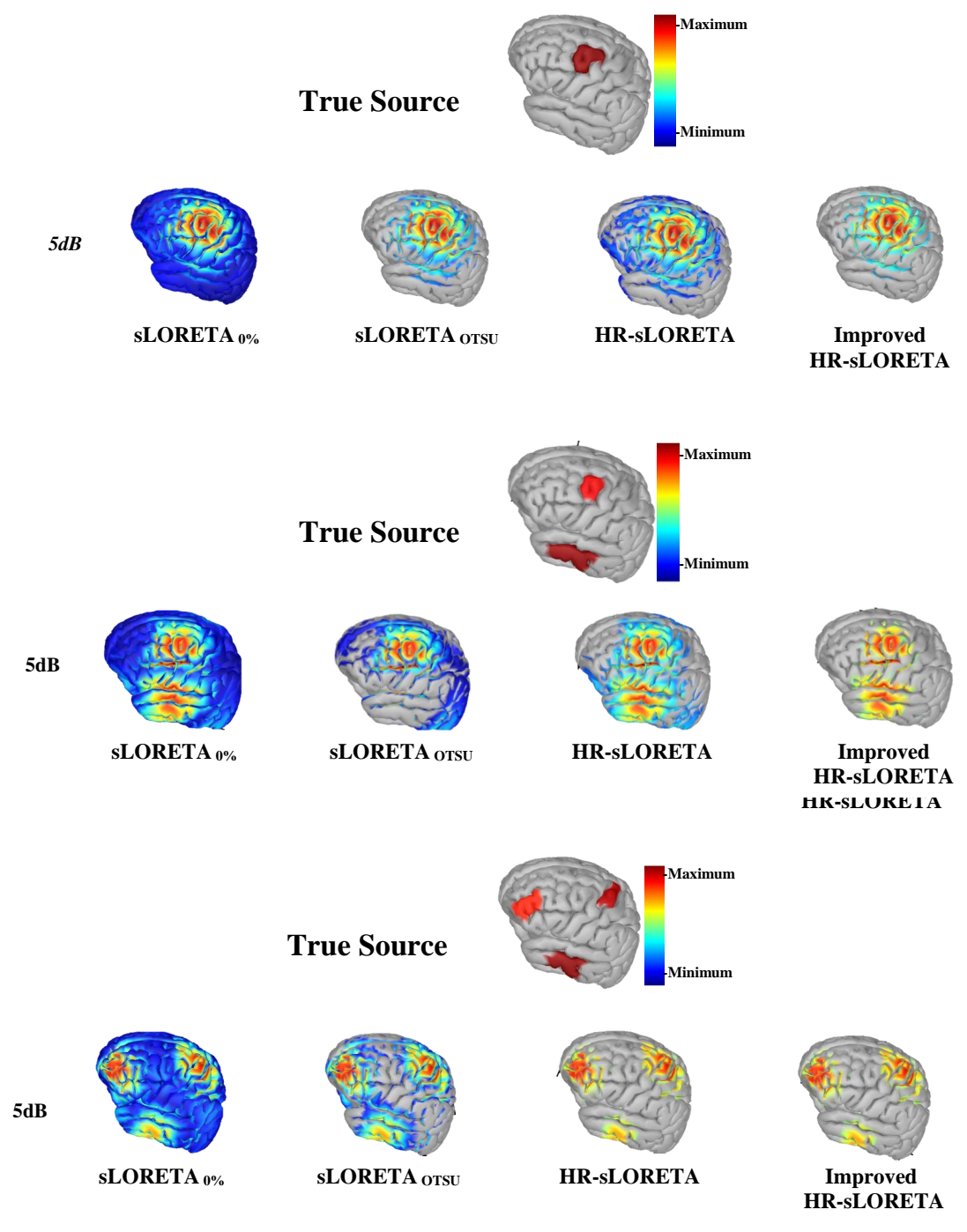

Figure 4.5: Quantitative comparison of Otsu method, HR-sLORETA, and Improved HR-sLORETA in SNR of $5 d B$ using one, two and three sources.

As is shown in the Figure 4.5, Improved HR-sLORETA illustrates the superior performance over the original HR-sLORETA method and Otsu method even at one source. Other than that, the quantitative analysis of the method is shown in Table 4.4 using SD metric. The lower value on this error shows better the performance (providing higher resolution) of the method. 
Table 4.4: SD in mm for SNR of $5 d B$

\begin{tabular}{|c||c||c||c||}
\hline Method & One Source & Two Source & Three Source \\
\hline 5dB & \multicolumn{3}{|c|}{} \\
\hline sLORETA $_{0 \%}$ & 1203.6 & 389.4526 & 142.9251 \\
sLORETA $_{\text {Otsu }}$ & 25.4421 & 194.1483 & 92.5864 \\
HR-sLORETA $_{\text {HR-sLORETA }}$ & 101.7381 & 70.3529 & 9.0719 \\
Improved & $\mathbf{2 3 . 1 3 3 1}$ & $\mathbf{6 . 8 8}$ & $\mathbf{5 . 7 6 6 7}$ \\
\hline
\end{tabular}

One must note that the simulation and result of this section are only provided to obtain an initial understanding about the performance of the method based on the proposed theory. To have an acceptable scientific conclusion about the superiority of the method over its competitions, a higher number of simulation and evaluation of the method under various conditions (different SNR values, different number of sources) are necessary. This is considered as future work of the method and explained in Chapter 5.

\subsection{Additional Observation on The Effect of the Added Con- straint in Regularization-Based models}

As described in Section 2.7, Regularization-based models are one of the popular frameworks within inverse solutions. While the first part of the equation $\left(\|M-H J\|_{2}\right)$ is a well-known error construction equation, the additional constraint on the right-hand side which is: $(\lambda f(J))$ is an added constraint. The objective of this short study is to investigate the influence of the added constraint on the result; particularly in the case of WMNE with $f(J)=\|J\|_{1}^{2}$. The purpose is to see, if this additional constraint is required due to the existence of the noise, or to provide a unique solution for the ill-posed problem. To analyze this question, the proposed source reconstruction method in [75] is used instead of the error $\|M-H J\|_{2}^{2}$ to find the value of $J$ without any additional constraint. This could be shown as follows:

$$
U(J)=\|M-H J\|_{2}^{2}+\lambda f(J) \stackrel{\text { Replaced with }}{\longrightarrow} U(J)=\bar{Z}_{m}(J)
$$

where $\bar{Z}_{m}(J)$ is the probabilistic upper bound of $Z(J)_{m}$ that is true source error:

$$
Z_{m}(J)=\left\|\bar{M}-H J_{m}\right\|_{2}^{2}
$$

Comparing $Z_{m}(J)$ with the error shown as $\left\|M-H J_{m}\right\|_{2}^{2}$, one could see that (4.23) considers the true noise-less error while $\|M-H J\|_{2}^{2}$ is the reconstruction error based on the noisy measurements $M$. If the minimization function $U(J)=\bar{Z}_{m}(J)$ represent acceptable source estimate, one could say that the additional constraint $f(J)$ is introduced to compensate for the existence of noise. On the other hand, if the source estimate from the minimization of (4.22) does not provide an acceptable result, it can be concluded that the additional constraint is to make the solution unique as in nature the problem 
is ill-posed and under-determined. While in theory, the lateral case is the hypothesis, I have put this problem under the test by obtaining the source estimate using the following formulation:

$$
\hat{J}=\arg \min _{m} \bar{Z}_{m}
$$

where simplification of $\bar{Z}_{m}$ is shown in the Appendix A.

In here, $X_{m}=\left\|M-H J_{m}\right\|_{2}^{2}$, where $J_{m}$ are the solutions of $J$ with $m$ number of non-zeroes.

Similar to the process that was explained in Chapter 4, two sources were simulated, and the performance of the source estimate using only $\bar{Z}_{m}$ as shown in (4.24) was evaluated. Figure 4.6 demonstrate the source estimate based on the equation defined in (4.24).

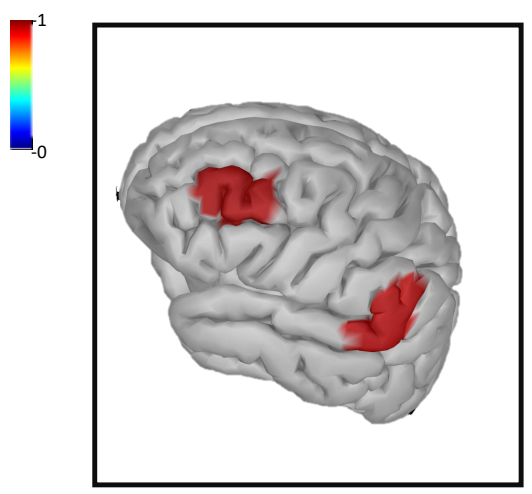

True Source

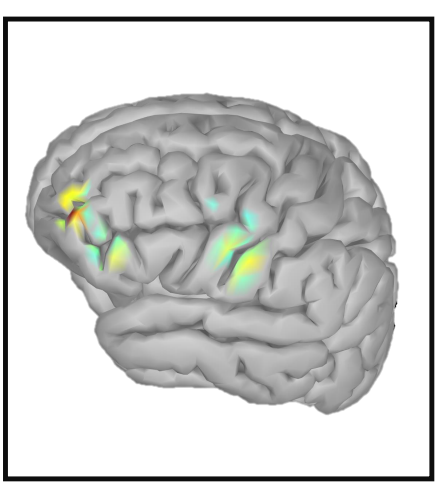

Source Estimate by (4.3)

Figure 4.6: Illustration of (4.24) performance on two synthetic sources.

As it is shown, the source estimate by (4.24) is not able to accurately estimate the location of the source. The illustrated result is one of the many solutions that can be obtained for this ill-posed problem. To be more precise, the error reconstruction by $Z_{m}$ is the solution with the least amount of additive WGN (due to the nature of $Z_{m}$ ); however, it is not the desired solution. The obtained results emphasize the importance of the additional constraint in estimating the correct solution. Among the introduced constraints, $L_{2}$ based solution (SLORETA in particular) has shown to provide an acceptable result with the additional constraint defined as weighted source energy (described in Section 2.7). However, the primary drawback of these solutions is having blurred and low resolution. Due to this reason, in the Section 4.1, a novel method is proposed, which improves the resolution of the $L_{2}$-based solutions without losing any important information or source. 


\section{Chapter 5}

\section{Conclusion and Implications for Future Research}

This thesis is focused on analyzing and processing brain source signals, which are obtained in the form of EEG and MEG measurements. EEG and MEG data both illustrate high temporal resolution while they have low spatial information. Moreover, EEG and MEG data are contaminated with additive noise and undesired biological signals, called artifacts. The objective of this thesis was to study the existing methods and propose new solutions that can potentially increase the efficiency of EEG and MEG data analysis. In this case, two main applications were introduced, first for estimating the number of components in ocular artifact removal (pre-processing); and second, to automate the process of thresholding in $L_{2}$-Regularization inverse solutions (post-processing).

In the case of pre-processing and denoising EEG and MEG measures, subspace artifact removal approaches, such as ICA, and SSP are taken into consideration. In order to utilize the subspace methods in the form of artifact removal, estimating the Number of Components (NoC) is required.

Unlike the conventional method where time-consuming analysis by an expert is required, in here, an automated process for estimating $\mathrm{NoC}$ is introduced, which is less dependent on the expertise input and more reliable. The proposed method is evaluated by both synthetic EEG data and real MEG data. The EEG-like synthetic data was used to evaluate the accuracy of the method in estimating NoC. This was done through the method of denoising EB artifacts using SSP, runICA and JADE subspace approaches. The obtained results illustrate the accuracy of $85.3 \%, 98.7 \%$ and $98.7 \%$ respectively. Moreover, an opensource MEG data was used to compare the proposed procedure and the manual estimation of NoC by data analysis method. The result of this section, illustrate that the proposed automated method can correctly detect the NoC as it was suggested in the manual procedure. While the proposed method and explanation in the thesis covers the mathematical and theoretical aspect of the method, to further evaluate the method, input of a clinical practitioner should be considered. Furthermore, to improve the proposed method, automating the selection of blinking and non-blinking time-frames, as well as sensitivity analysis of the method on the number of time samples required from blinking and the non- 
blinking region is suggested.

The second part of the thesis, which is introduced in the form of post-processing, considers the source localization techniques used in EEG source imaging. In particular, popular L2-Regularization inverse solutions are studies in details. In order to improve the state-of-art inverse solutions, a post-processing method in the form of automatic thresholding is introduced. However, the major problem with $L_{2}$-based inverse solutions is that they have a low resolution; nevertheless, the proposed method could denoise the source estimate to further increase their resolution without losing neither important information nor source.

The method is further improved and focused on SLORETA, which is the most popular inverse solution. This improvement is made by introducing more accurate noise variance estimation, which is the requirement of the proposed thresholding method. The method is evaluated by using synthetic EEG data for the different number of sources and different SNR values. This assessment is done by using wellestablished evaluation metrics such as Percentage of Undetected Sources (PUS) and Spatial Dispersion (SD). The proposed noise variance estimation utilizes entropy and kurtosis of the obtained noise to construct an error function. However, in the current algorithm, the influence of each two parameters are with the same weight. The method can further develop and improve by adding a weight to each evaluation parameter and sets a trade-off for the reliance of error on entropy and kurtosis. To ensure the performance of the new noise variance estimate, a higher number of simulation is required. Moreover, the use of real EEG data in the simulation could also be considered for the future work of the project. 
Appendices 


\section{Appendix A}

\section{Mathematical Simplification of The Error Reconstruction Algorithm}

In this appendix, the mathematical simplification on the upper bound of reconstruction error $\left(Z_{m}\right)$, proposed in [75], is provided. This simplification leads to $3 \pm 1$ seconds improvement in the speed of the algorithm computational time (averaged over 20 trials), which was necessary for the proposed automatic thresholding.

The following (A.1) is the equation (62) of [75], which will be used as the starting point of the simplification:

$$
\bar{Z}_{m}=\frac{m}{N} \sigma_{\omega}^{2}+U_{m}+\beta \frac{\sqrt{2 m}}{N} \sigma_{\omega}^{2}
$$

where $U_{m}$ is illustrated on (50) of [75] as the following:

$$
U_{m}=X_{m}-m_{\omega}+\frac{2 \alpha^{2} \sigma_{\omega}^{2}}{N}+K_{m}
$$

where $m_{\omega}$ and $K_{m}$ are shown in (52) and (51) of [75] respecitvely as:

$$
\begin{gathered}
m_{\omega}=\left(1-\frac{m}{N}\right) \sigma_{\omega}^{2} \\
K_{m}=2 \alpha \frac{\sigma_{\omega}}{\sqrt{N}} \sqrt{\frac{\alpha^{2} \sigma_{\omega}^{2}}{N}+X_{m}-\frac{1}{2} m_{\omega}}
\end{gathered}
$$

Let us start with expanding (A.1) by substituting (A.2) in the equation:

$$
\bar{Z}_{m}=X_{m}-m_{\omega}+\frac{2 \alpha^{2} \sigma_{\omega}^{2}}{N}+K_{m}+\frac{m}{N} \sigma_{\omega}^{2}+\beta \frac{\sqrt{2 m}}{N} \sigma_{\omega}^{2}
$$


Moreover, substituting (A.3) into (A.5) will lead to:

$$
\bar{Z}_{m}=X_{m}-\left(1-\frac{m}{N}\right) \sigma_{\omega}^{2}+\frac{2 \alpha^{2}}{N} \sigma_{\omega}^{2}+K_{m}+\frac{m}{N} \sigma_{\omega}^{2}+\beta \frac{\sqrt{2 m}}{N} \sigma_{\omega}^{2}
$$

Re-arranging the equation and expanding the $m_{\omega}$ bracket will lead to:

$$
\bar{Z}_{m}=X_{m}-\sigma_{\omega}^{2}+\frac{m}{N} \sigma_{\omega}^{2}+\frac{2 \alpha^{2}}{N} \sigma_{\omega}^{2}+\frac{m}{N} \sigma_{\omega}^{2}+\beta \frac{\sqrt{2 m}}{N} \sigma_{\omega}^{2}+K_{m}
$$

Factoring-out $\frac{\sigma_{\omega}^{2}}{N}$ will result in:

$$
\bar{Z}_{m}=X_{m}+\left(\beta \sqrt{2 m}+2 m+2 \alpha^{2}-N\right) \frac{\sigma_{\omega}^{2}}{N}+K_{m}
$$

Substituting (A.4) into (A.8) is:

$$
\bar{Z}_{m}=X_{m}+\left(\beta \sqrt{2 m}+2 m-N+2 \alpha^{2}\right) \frac{\sigma_{\omega}^{2}}{N}+2 \alpha \frac{\sigma_{\omega}}{\sqrt{N}} \sqrt{\frac{\alpha^{2} \sigma_{\omega}^{2}}{N}+X_{m}-\frac{1}{2} m_{\omega}}
$$

The most right hand side equation which is $K_{m}$ could be multiplied and divided by $\frac{\sigma_{\omega}}{\sqrt{N}}$ :

$$
\bar{Z}_{m}=X_{m}+\left(\beta \sqrt{2 m}+2 m-N+2 \alpha^{2}\right) \frac{\sigma_{\omega}^{2}}{N}+\frac{\sigma_{\omega}}{\sqrt{N}}\left(\frac{\sigma_{\omega}}{\sqrt{N}} \times \frac{\sqrt{N}}{\sigma_{\omega}}\right) 2 \alpha \sqrt{\frac{\alpha^{2} \sigma_{\omega}^{2}}{N}+X_{m}-\frac{1}{2} m_{\omega}}
$$

Simplifying (A.10) will lead to:

$$
\bar{Z}_{m}=X_{m}+\left(\beta \sqrt{2 m}+2 m-N+2 \alpha^{2}\right) \frac{\sigma_{\omega}^{2}}{N}+\frac{\sigma_{\omega}^{2}}{N}\left(\frac{\sqrt{N}}{\sigma_{\omega}}\right) 2 \alpha \sqrt{\frac{\alpha^{2} \sigma_{\omega}^{2}}{N}+X_{m}-\frac{1}{2} m_{\omega}}
$$

which is equal to:

$$
\bar{Z}_{m}=X_{m}+\left(\beta \sqrt{2 m}+2 m-N+2 \alpha^{2}\right) \frac{\sigma_{\omega}^{2}}{N}+\frac{\sigma_{\omega}^{2}}{N} 2 \alpha \frac{\sqrt{\alpha^{2} \sigma_{\omega}^{2}+N X_{m}-\frac{N}{2} m_{\omega}}}{\sigma_{\omega}}
$$

Factorization with respect to $\frac{\sigma_{\omega}^{2}}{N}$ will lead to:

$$
\bar{Z}_{m}=X_{m}+\left(\beta \sqrt{2 m}+2 m-N+2 \alpha^{2}+2 \alpha \frac{\sqrt{\alpha^{2} \sigma_{\omega}^{2}+N X_{m}-\frac{N}{2} m_{\omega}}}{\sigma_{\omega}}\right) \frac{\sigma_{\omega}^{2}}{N}
$$

Finally, factoring $2 \alpha$ from the equation in the bracket will result in:

$$
\bar{Z}_{m}=X_{m}+\left(\beta \sqrt{2 m}+2 m-N+2 \alpha\left(\alpha+\frac{\sqrt{\alpha^{2} \sigma_{\omega}^{2}+N X_{m}-\frac{N}{2} m_{\omega}}}{\sigma_{\omega}}\right)\right) \frac{\sigma_{\omega}^{2}}{N}
$$




\section{References}

[1] O. J. Arthurs and S. Boniface, "How well do we understand the neural origins of the fMRI BOLD signal?" Trends Neurosci., vol. 25, no. 1, pp. 27-31, Jan 2002.

[2] A. K. Engel, P. Fries, and W. Singer, "Dynamic predictions: oscillations and synchrony in topdown processing," Nature Reviews Neuroscience, vol. 2, no. 10, p. 704, 2001.

[3] K. Friston, C. Frith, P. Liddle, and R. Frackowiak, "Functional connectivity: the principalcomponent analysis of large (pet) data sets," Journal of Cerebral Blood Flow \& Metabolism, vol. 13, no. 1 , pp. 5-14, 1993.

[4] P. van Mierlo, M. Papadopoulou, E. Carrette, P. Boon, S. Vandenberghe, K. Vonck, and D. Marinazzo, "Functional brain connectivity from eeg in epilepsy: Seizure prediction and epileptogenic focus localization," Progress in neurobiology, vol. 121, pp. 19-35, 2014.

[5] J. Ebersole, "Non-invasive localization of the epileptogenic focus by eeg dipole modeling," Acta Neurologica Scandinavica, vol. 89, no. S152, pp. 20-28, 1994.

[6] B. Reisberg, L. Prichep, L. Mosconi, E. R. John, L. Glodzik-Sobanska, I. Boksay, I. Monteiro, C. Torossian, A. Vedvyas, N. Ashraf et al., "The pre-mild cognitive impairment, subjective cognitive impairment stage of alzheimers disease," 2008.

[7] R. V. Sheorajpanday, P. Marien, A. J. Weeren, G. Nagels, J. Saerens, M. J. van Putten, and P. P. De Deyn, "Eeg in silent small vessel disease: sloreta mapping reveals cortical sources of vascular cognitive impairment no dementia in the default mode network," Journal of clinical neurophysiology, vol. 30, no. 2, pp. 178-187, 2013.

[8] Y. Tang, X. Zhang, M. Simmonite, H. Li, T. Zhang, Q. Guo, C. Li, Y. Fang, Y. Xu, and J. Wang, "Hyperactivity within an extensive cortical distribution associated with excessive sensitivity in error processing in unmedicated depression: a combined event-related potential and sloreta study," International Journal of Psychophysiology, vol. 90, no. 2, pp. 282-289, 2013.

[9] P. Flor-Henry, J. C. Lind, and Z. J. Koles, "A source-imaging (low-resolution electromagnetic tomography) study of the eegs from unmedicated males with depression," Psychiatry Research: Neuroimaging, vol. 130, no. 2, pp. 191-207, 2004. 
[10] L. Canuet, R. Ishii, R. D. Pascual-Marqui, M. Iwase, R. Kurimoto, Y. Aoki, S. Ikeda, H. Takahashi, T. Nakahachi, and M. Takeda, "Resting-state eeg source localization and functional connectivity in schizophrenia-like psychosis of epilepsy," PloS one, vol. 6, no. 11, p. e27863, 2011.

[11] M. Reite, J. Sheeder, P. Teale, M. Adams, D. Richardson, J. Simon, R. H. Jones, and D. C. Rojas, "Magnetic source imaging evidence of sex differences in cerebral lateralization in schizophrenia," Archives of General Psychiatry, vol. 54, no. 5, pp. 433-440, 1997.

[12] Z. J. Koles, J. C. Lind, and P. Flor-Henry, "A source-imaging (low-resolution electromagnetic tomography) study of the eegs from unmedicated men with schizophrenia," Psychiatry Research: Neuroimaging, vol. 130, no. 2, pp. 171-190, 2004.

[13] R. C. Knowlton, R. Elgavish, J. Howell, J. Blount, J. G. Burneo, E. Faught, P. Kankirawatana, K. Riley, R. Morawetz, J. Worthington et al., "Magnetic source imaging versus intracranial electroencephalogram in epilepsy surgery: a prospective study," Annals of Neurology: Official Journal of the American Neurological Association and the Child Neurology Society, vol. 59, no. 5, pp. 835-842, 2006.

[14] G. Michelini, J. Jurgiel, I. Bakolis, C. H. Cheung, P. Asherson, S. K. Loo, J. Kuntsi, and I. Mohammad-Rezazadeh, "6.29 atypical functional connectivity in adolescents and adults with persistent and remitted attention-deficit/hyperactivity disorder (adhd)," Journal of the American Academy of Child \&3 Adolescent Psychiatry, vol. 56, no. 10, p. S286, 2017.

[15] K. Patton, Anatomy and Physiology - E-Book. Elsevier Health Sciences, 2015. [Online]. Available: https://books.google.ca/books?id=Ko2bBgAAQBAJ

[16] M. Piccolino, "Luigi galvani and animal electricity: two centuries after the foundation of electrophysiology," Trends in neurosciences, vol. 20, no. 10, pp. 443-448, 1997.

[17] J. Vrba and S. E. Robinson, "Signal processing in magnetoencephalography," Methods, vol. 25, no. 2, pp. 249-271, 2001.

[18] N. K. Munsif Ali Jatoi, Brain Source Localization Using EEG Signal Analysis, 1st ed. CRC Press, 2018.

[19] D. Cohen, "Magnetoencephalography: detection of the brain's electrical activity with a superconducting magnetometer," Science, vol. 175, no. 4022, pp. 664-666, 1972.

[20] W. Barbosa, "Making of 'soldier'," Jun 2009. [Online]. Available: https://3dtotal.com/tutorials/ t/making-of-soldier-wesclei-barbosa-character-man-human-soldier-skull-helmet

[21] M. X. Cohen, Analyzing neural time series data: theory and practice. MIT press, 2014.

[22] P. Comon and C. Jutten, Handbook of Blind Source Separation: Independent component analysis and applications. Academic press, 2010. 
[23] T.-P. Jung, S. Makeig, C. Humphries, T.-W. Lee, M. J. Mckeown, V. Iragui, and T. J. Sejnowski, "Removing electroencephalographic artifacts by blind source separation," Psychophysiology, vol. 37, no. 2, pp. 163-178, 2000.

[24] G. R. Naik, W. Wang et al., "Blind source separation," Berlin: Springer, vol. 10, pp. 978-3, 2014.

[25] A. Varsavsky, I. Mareels, and M. Cook, Epileptic seizures and the EEG: measurement, models, detection and prediction. CRC Press, 2016.

[26] G. H. Klem, H. O. Lüders, H. Jasper, C. Elger et al., "The ten-twenty electrode system of the international federation," Electroencephalogr Clin Neurophysiol, vol. 52, no. 3, pp. 3-6, 1999.

[27] — , "The ten-twenty electrode system of the international federation," Electroencephalogr Clin Neurophysiol, vol. 52, no. 3, pp. 3-6, 1999.

[28] F. Tadel, S. Baillet, J. C. Mosher, D. Pantazis, and R. M. Leahy, "Brainstorm: a user-friendly application for meg/eeg analysis," Computational intelligence and neuroscience, vol. 2011, p. 8, 2011.

[29] A. Delorme and S. Makeig, "Eeglab: an open source toolbox for analysis of single-trial eeg dynamics including independent component analysis," Journal of neuroscience methods, vol. 134, no. 1, pp. 9-21, 2004.

[30] H. Adeli and S. Ghosh-Dastidar, Automated EEG-based diagnosis of neurological disorders: Inventing the future of neurology. CRC press, 2010.

[31] A. Hyvärinen and E. Oja, "Independent component analysis: algorithms and applications," Neural networks, vol. 13, no. 4-5, pp. 411-430, 2000.

[32] J.-F. Cardoso and A. Souloumiac, "Blind beamforming for non-gaussian signals," in IEE proceedings $F$ (radar and signal processing), vol. 140, no. 6. IET, 1993, pp. 362-370.

[33] T. M. Cover and J. A. Thomas, Elements of information theory. John Wiley \& Sons, 2012.

[34] A. Hyvärinen, "New approximations of differential entropy for independent component analysis and projection pursuit," in Advances in neural information processing systems, 1998, pp. 273-279.

[35] M. C. Jones and R. Sibson, "What is projection pursuit?" Journal of the Royal Statistical Society: Series A (General), vol. 150, no. 1, pp. 1-18, 1987.

[36] T.-P. Jung, C. Humphries, T.-W. Lee, S. Makeig, M. J. McKeown, V. Iragui, and T. J. Sejnowski, "Extended ica removes artifacts from electroencephalographic recordings," in Advances in neural information processing systems, 1998, pp. 894-900.

[37] C. Tesche, M. Uusitalo, R. Ilmoniemi, M. Huotilainen, M. Kajola, and O. Salonen, "Signal-space projections of meg data characterize both distributed and well-localized neuronal sources," Electroencephalography and clinical neurophysiology, vol. 95, no. 3, pp. 189-200, 1995. 
[38] M. A. Uusitalo and R. J. Ilmoniemi, "Signal-space projection method for separating meg or eeg into components," Medical and Biological Engineering and Computing, vol. 35, no. 2, pp. 135-140, 1997.

[39] G. Kang, S.-H. Jin, D. K. Kim, and S. W. Kang, "T59. eeg artifacts removal using machine learning algorithms and independent component analysis," Clinical Neurophysiology, vol. 129, p. e24, 2018.

[40] S.-Y. Shao, K.-Q. Shen, C. J. Ong, E. P. Wilder-Smith, and X.-P. Li, "Automatic eeg artifact removal: a weighted support vector machine approach with error correction," IEEE Transactions on Biomedical Engineering, vol. 56, no. 2, pp. 336-344, 2008.

[41] A. Craik, Y. He, and J. L. Contreras-Vidal, "Deep learning for electroencephalogram (eeg) classification tasks: a review," Journal of neural engineering, vol. 16, no. 3, p. 031001, 2019.

[42] P. Nejedly, J. Cimbalnik, P. Klimes, F. Plesinger, J. Halamek, V. Kremen, I. Viscor, B. H. Brinkmann, M. Pail, M. Brazdil et al., "Intracerebral eeg artifact identification using convolutional neural networks," Neuroinformatics, vol. 17, no. 2, pp. 225-234, 2019.

[43] B. Somers, T. Francart, and A. Bertrand, "A generic eeg artifact removal algorithm based on the multi-channel wiener filter," Journal of neural engineering, vol. 15, no. 3, p. 036007, 2018.

[44] N. Mammone, F. La Foresta, and F. C. Morabito, "Automatic artifact rejection from multichannel scalp eeg by wavelet ica," IEEE Sensors Journal, vol. 12, no. 3, pp. 533-542, 2011.

[45] H. Becker, "Denoising, separation and localization of eeg sources in the context of epilepsy," Ph.D. dissertation, Université Nice Sophia Antipolis, 2014.

[46] P. Comon, "Independent component analysis, a new concept?" Signal processing, vol. 36, no. 3, pp. 287-314, 1994.

[47] R. Grech, T. Cassar, J. Muscat, K. P. Camilleri, S. G. Fabri, M. Zervakis, P. Xanthopoulos, V. Sakkalis, and B. Vanrumste, "Review on solving the inverse problem in eeg source analysis," Journal of neuroengineering and rehabilitation, vol. 5, no. 1, p. 25, 2008.

[48] J. X. Tao, A. Ray, S. Hawes-Ebersole, and J. S. Ebersole, "Intracranial eeg substrates of scalp eeg interictal spikes," Epilepsia, vol. 46, no. 5, pp. 669-676, 2005.

[49] I. Merlet and J. Gotman, "Reliability of dipole models of epileptic spikes," Clinical neurophysiology, vol. 110, no. 6, pp. 1013-1028, 1999.

[50] J. S. Ebersole, "Magnetoencephalography/magnetic source imaging in the assessment of patients with epilepsy," Epilepsia, vol. 38, pp. S1-S5, 1997.

[51] M. Gavaret, J.-M. Badier, P. Marquis, A. McGonigal, F. Bartolomei, J. Regis, and P. Chauvel, "Electric source imaging in frontal lobe epilepsy," Journal of Clinical Neurophysiology, vol. 23, no. 4, pp. 358-370, 2006. 
[52] S. Baillet, J. C. Mosher, and R. M. Leahy, "Electromagnetic brain mapping," IEEE Signal Processing Magazine, vol. 18, no. 6, pp. 14-30, Nov 2001.

[53] J. C. Mosher, R. M. Leahy, and P. S. Lewis, "Eeg and meg: forward solutions for inverse methods," IEEE Transactions on Biomedical Engineering, vol. 46, no. 3, pp. 245-259, 1999.

[54] B. N. Cuffin and D. Cohen, "Magnetic fields of a dipole in special volume conductor shapes," IEEE Transactions on Biomedical Engineering, no. 4, pp. 372-381, 1977.

[55] J. Sarvas, "Basic mathematical and electromagnetic concepts of the biomagnetic inverse problem," Physics in Medicine 83 Biology, vol. 32, no. 1, p. 11, 1987.

[56] D. A. Brody, F. H. Terry, and R. E. Ideker, "Eccentric dipole in a spherical medium: generalized expression for surface potentials," IEEE Transactions on Biomedical Engineering, no. 2, pp. 141143, 1973.

[57] S. Næss, C. Chintaluri, T. V. Ness, A. M. Dale, G. T. Einevoll, and D. K. Wójcik, "Corrected four-sphere head model for eeg signals," Frontiers in human neuroscience, vol. 11, p. 490, 2017.

[58] M. Fuchs, R. Drenckhahn, H. Wischmann, and M. Wagner, "An improved boundary element method for realistic volume-conductor modeling," IEEE Transactions on Biomedical Engineering, vol. 45, no. 8, pp. 980-997, 1998.

[59] Y. Yan, P. Nunez, and R. Hart, "Finite-element model of the human head: scalp potentials due to dipole sources," Medical and Biological Engineering and Computing, vol. 29, no. 5, pp. 475-481, 1991.

[60] H. Hallez, B. Vanrumste, P. Van Hese, Y. D'Asseler, I. Lemahieu, and R. Van de Walle, "A finite difference method with reciprocity used to incorporate anisotropy in electroencephalogram dipole source localization," Physics in Medicine $\&$ Biology, vol. 50, no. 16, p. 3787, 2005.

[61] H. Becker, L. Albera, P. Comon, R. Gribonval, F. Wendling, and I. Merlet, "Brain-source imaging: From sparse to tensor models," IEEE Signal Processing Magazine, vol. 32, no. 6, pp. 100-112, 2015.

[62] A. K. Liu, A. M. Dale, and J. W. Belliveau, "Monte carlo simulation studies of eeg and meg localization accuracy," Human brain mapping, vol. 16, no. 1, pp. 47-62, 2002.

[63] M. Hmlinen and R. Ilmoniemi, "Interpreting measured magnetic fields of the brain: Estimates of current distributions," vol. 32, 121984.

[64] P. C. Hansen, Rank-deficient and discrete ill-posed problems: numerical aspects of linear inversion. Siam, 2005, vol. 4 .

[65] R. D. Pascual-Marqui, "Review of methods for solving the eeg inverse problem," International journal of bioelectromagnetism, vol. 1, no. 1, pp. 75-86, 1999. 
[66] R. Pascual-Marqui, C. Michel, and D. Lehmann, "Low resolution electromagnetic tomography: a new method for localizing electrical activity in the brain," International Journal of Psychophysiology, vol. 18, no. 1, pp. 49 - 65, 1994. [Online]. Available: http: //www.sciencedirect.com/science/article/pii/016787608490014X

[67] A. M. Dale, A. K. Liu, B. R. Fischl, R. L. Buckner, J. W. Belliveau, J. D. Lewine, and E. Halgren, "Dynamic statistical parametric mapping: combining fmri and meg for high-resolution imaging of cortical activity," Neuron, vol. 26, no. 1, pp. 55-67, 2000.

[68] R. D. Pascual-Marqui et al., "Standardized low-resolution brain electromagnetic tomography (sloreta): technical details," Methods Find Exp Clin Pharmacol, vol. 24, no. Suppl D, pp. 5-12, 2002 .

[69] K. Matsuura and Y. Okabe, "A robust reconstruction of sparse biomagnetic sources," IEEE Transactions on Biomedical Engineering, vol. 44, no. 8, pp. 720-726, 1997.

[70] L. Ding and B. He, "Sparse source imaging in electroencephalography with accurate field modeling," Human brain mapping, vol. 29, no. 9, pp. 1053-1067, 2008.

[71] I. F. Gorodnitsky, J. S. George, and B. D. Rao, "Neuromagnetic source imaging with focuss: a recursive weighted minimum norm algorithm," Electroencephalography and clinical Neurophysiology, vol. 95, no. 4, pp. 231-251, 1995.

[72] W. Ou, M. S. Hämäläinen, and P. Golland, "A distributed spatio-temporal eeg/meg inverse solver," NeuroImage, vol. 44, no. 3, pp. 932-946, 2009.

[73] W. Deburchgraeve, P. J. Cherian, M. De Vos, R. M. Swarte, J. H. Blok, G. H. Visser, P. Govaert, and S. Van Huffel, "Neonatal seizure localization using parafac decomposition," Clinical Neurophysiology, vol. 120, no. 10, pp. 1787-1796, 2009.

[74] H. Becker, L. Albera, P. Comon, M. Haardt, G. Birot, F. Wendling, M. Gavaret, C. G. Bénar, and I. Merlet, "Eeg extended source localization: tensor-based vs. conventional methods," NeuroImage, vol. 96, pp. 143-157, 2014.

[75] S. Beheshti and M. A. Dahleh, "A new information-theoretic approach to signal denoising and best basis selection," IEEE Transactions on Signal Processing, vol. 53, no. 10, pp. 3613-3624, 2005.

[76] S. Beheshti and S. Sedghizadeh, "Number of source signal estimation by the mean squared eigenvalue error," IEEE Transactions on Signal Processing, vol. 66, no. 21, pp. 5694-5704, 2018.

[77] M. Fatourechi, A. Bashashati, R. K. Ward, and G. E. Birch, "Emg and eog artifacts in brain computer interface systems: A survey," Clinical neurophysiology, vol. 118, no. 3, pp. 480-494, 2007.

[78] R. J. Croft and R. J. Barry, "Removal of ocular artifact from the eeg: a review," Neurophysiologie Clinique/Clinical Neurophysiology, vol. 30, no. 1, pp. 5-19, 2000. 
[79] C. Jutten and J. Herault, "Blind separation of sources, part i: An adaptive algorithm based on neuromimetic architecture," Signal processing, vol. 24, no. 1, pp. 1-10, 1991.

[80] B. Noureddin, P. D. Lawrence, and G. E. Birch, "Quantitative evaluation of ocular artifact removal methods based on real and estimated eog signals," in 2008 30th Annual International Conference of the IEEE Engineering in Medicine and Biology Society. IEEE, 2008, pp. 5041-5044.

[81] S. Sreeja, R. R. Sahay, D. Samanta, and P. Mitra, "Removal of eye blink artifacts from eeg signals using sparsity," IEEE journal of biomedical and health informatics, vol. 22, no. 5, pp. 1362-1372, 2018.

[82] D. Overton and C. Shagass, "Distribution of eye movement and eyeblink potentials over the scalp." Electroencephalography and Clinical Neurophysiology, vol. 27, no. 5, pp. 546-546, 1969.

[83] D. Hagemann and E. Naumann, "The effects of ocular artifacts on (lateralized) broadband power in the eeg," Clinical Neurophysiology, vol. 112, no. 2, pp. 215-231, 2001.

[84] M. Chaumon, D. V. Bishop, and N. A. Busch, "A practical guide to the selection of independent components of the electroencephalogram for artifact correction," Journal of neuroscience methods, vol. 250, pp. 47-63, 2015.

[85] P. Berg and M. Scherg, "Dipole modelling of eye activity and its application to the removal of eye artefacts from the eeg and meg," Clinical Physics and Physiological Measurement, vol. 12, no. A, p. 49, 1991.

[86] J. C. Mosher, R. M. Leahy, and P. S. Lewis, "Eeg and meg: forward solutions for inverse methods," IEEE Transactions on Biomedical Engineering, vol. 46, no. 3, pp. 245-259, 1999.

[87] T.-P. Jung, C. Humphries, T.-W. Lee, S. Makeig, M. J. McKeown, V. Iragui, and T. J. Sejnowski, "Extended ica removes artifacts from electroencephalographic recordings," in Advances in neural information processing systems, 1998, pp. 894-900.

[88] F. Tadel, E. A. Bock, G. Niso, J. C. Mosher, M. Cousineau, D. Pantazis, R. M. Leahy, and S. Baillet, "Meg/eeg group analysis with brainstorm," Frontiers in neuroscience, vol. 13, p. 76, 2019.

[89] C. Burger and D. J. van den Heever, "Removal of eog artefacts by combining wavelet neural network and independent component analysis," Biomedical Signal Processing and Control, vol. 15, pp. 67-79, 2015.

[90] D. J. McFarland, L. M. McCane, S. V. David, and J. R. Wolpaw, "Spatial filter selection for eeg-based communication," Electroencephalography and clinical Neurophysiology, vol. 103, no. 3, pp. 386-394, 1997. 
[91] A. M. Dale and M. I. Sereno, "Improved localizadon of cortical activity by combining eeg and meg with mri cortical surface reconstruction: a linear approach," Journal of cognitive neuroscience, vol. 5, no. 2, pp. 162-176, 1993.

[92] M. Hämäläinen, "Mne software user's guide," NMR Center, Mass General Hospital, Harvard University, vol. 58, pp. 59-75, 2005.

[93] D. A. Engemann and A. Gramfort, "Automated model selection in covariance estimation and spatial whitening of meg and eeg signals," NeuroImage, vol. 108, pp. 328-342, 2015.

[94] S. Beheshti and M. A. Dahleh, "A new information-theoretic approach to signal denoising and best basis selection," IEEE Transactions on Signal Processing, vol. 53, no. 10, pp. 3613-3624, 2005.

[95] S. Beheshti, "Kullback-leibler distance in linear parametric modeling," in 2008 IEEE International Symposium on Information Theory, July 2008, pp. 1671-1675.

[96] S. Beheshti and M. A. Dahleh, "Noisy data and impulse response estimation," IEEE Transactions on Signal Processing, vol. 58, no. 2, pp. 510-521, 2010.

[97] K. Liu, Z. Yu, W. Wu, Z. Gu, J. Zhang, L. Cen, S. Nagarajan, and Y. Li, "Bayesian electromagnetic spatio-temporal imaging of extended sources based on matrix factorization." IEEE transactions on bio-medical engineering, 2019.

[98] Y. Huang, L. C. Parra, and S. Haufe, "The new york heada precise standardized volume conductor model for eeg source localization and tes targeting," NeuroImage, vol. 140, pp. 150-162, 2016.

[99] J. C. Mosher, R. M. Leahy, and P. S. Lewis, "Eeg and meg: forward solutions for inverse methods," IEEE Transactions on Biomedical Engineering, vol. 46, no. 3, pp. 245-259, 1999.

[100] A. Sohrabpour, Y. Lu, G. Worrell, and B. He, "Imaging brain source extent from eeg/meg by means of an iteratively reweighted edge sparsity minimization (ires) strategy," NeuroImage, vol. 142, pp. 27-42, 2016.

[101] J.-H. Cho, S. B. Hong, Y.-J. Jung, H.-C. Kang, H. D. Kim, M. Suh, K.-Y. Jung, and C.-H. Im, "Evaluation of algorithms for intracranial eeg (ieeg) source imaging of extended sources: feasibility of using ieeg source imaging for localizing epileptogenic zones in secondary generalized epilepsy," Brain topography, vol. 24, no. 2, pp. 91-104, 2011.

[102] C. Li, D. Jacobs, T. Hilton, M. Del Campo, Y. Chinvarun, P. L. Carlen, and B. L. Bardakjian, "Epileptogenic source imaging using cross-frequency coupled signals from scalp eeg," IEEE Transactions on Biomedical Engineering, vol. 63, no. 12, pp. 2607-2618, 2016.

[103] C. Grova, J. Daunizeau, J.-M. Lina, C. Bénar, H. Benali, and J. Gotman, "Evaluation of eeg localization methods using realistic simulations of interictal spikes," Neuroimage, vol. 29, no. 3, pp. $734-753,2006$. 
[104] J. Yao and J. P. Dewald, "Evaluation of different cortical source localization methods using simulated and experimental eeg data," Neuroimage, vol. 25, no. 2, pp. 369-382, 2005.

[105] L. T. DeCarlo, "On the meaning and use of kurtosis." Psychological methods, vol. 2, no. 3, p. 292, 1997.

[106] C. J. Holmes, R. Hoge, L. Collins, R. Woods, A. W. Toga, and A. C. Evans, "Enhancement of mr images using registration for signal averaging," Journal of computer assisted tomography, vol. 22, no. 2, pp. 324-333, 1998.

[107] M. Huang, J. C. Mosher, and R. Leahy, "A sensor-weighted overlapping-sphere head model and exhaustive head model comparison for meg," Physics in Medicine 6 Biology, vol. 44, no. 2, p. 423, 1999.

[108] A. Sohrabpour, Y. Lu, G. Worrell, and B. He, "Imaging brain source extent from eeg/meg by means of an iteratively reweighted edge sparsity minimization (ires) strategy," NeuroImage, vol. 142, pp. 27-42, 2016.

[109] J.-H. Cho, S. B. Hong, Y.-J. Jung, H.-C. Kang, H. D. Kim, M. Suh, K.-Y. Jung, and C.-H. Im, "Evaluation of algorithms for intracranial eeg (ieeg) source imaging of extended sources: feasibility of using ieeg source imaging for localizing epileptogenic zones in secondary generalized epilepsy," Brain topography, vol. 24, no. 2, pp. 91-104, 2011.

[110] C. Li, D. Jacobs, T. Hilton, M. Del Campo, Y. Chinvarun, P. L. Carlen, and B. L. Bardakjian, "Epileptogenic source imaging using cross-frequency coupled signals from scalp eeg," IEEE Transactions on Biomedical Engineering, vol. 63, no. 12, pp. 2607-2618, 2016.

[111] N. Otsu, "A threshold selection method from gray-level histograms," IEEE transactions on systems, man, and cybernetics, vol. 9, no. 1, pp. 62-66, 1979.

[112] K. Liu, Z. L. Yu, W. Wu, Z. Gu, Y. Li, and S. Nagarajan, "Variation sparse source imaging based on conditional mean for electromagnetic extended sources," Neurocomputing, vol. 313, pp. 96-110, 2018.

[113] J. Yao and J. P. Dewald, "Evaluation of different cortical source localization methods using simulated and experimental eeg data," Neuroimage, vol. 25, no. 2, pp. 369-382, 2005.

[114] A. Molins, S. M. Stufflebeam, E. N. Brown, and M. S. Hämäläinen, "Quantification of the benefit from integrating meg and eeg data in minimum 12-norm estimation," Neuroimage, vol. 42, no. 3, pp. 1069-1077, 2008.

[115] Z. Drzazga, K. Maciejewska, K. Banasiak, I. Karpiel, and A. Giec-Lorenz, "Visual language task in eeg source localization analysis and functional mri," in Image and Signal Processing, BioMedical Engineering and Informatics (CISP-BMEI), International Congress on. IEEE, 2016, pp. 974-978. 
[116] D. Zhang, Y. Zhou, and J. Yuan, "Speech prosodies of different emotional categories activate different brain regions in adult cortex: an fnirs study," Scientific reports, vol. 8, no. 1, p. 218, 2018.

[117] R. Rojas, "Why the normal distribution," Freis Universitat Berlin lecture notes, 2010.

[118] S. L. Eddins, R. Gonzalez, and R. Woods, "Digital image processing using matlab," Princeton Hall Pearson Education Inc., New Jersey, 2004. 


\section{Acronyms}

AESET Automated EEG Source Error Thresholding. ix, 39-45

BEM Boundary Element Method. ix, 22

BSS Blind Source Separation. 10, 12

dSPM dynamic Statistical Parametric Mapping. 24, 41

EB Eye Blinking. 15, 28, 29, 56

ECD Equivalent Current Dipole. 17

EEG Electroencephalography. vii, ix, 1, 2, 6-12, 15, 16, 18, 21, 23, 24, 28-34, 39, 46, 56, 57

EmpB Emperical Bayesian. 26

EOG ElectroOculoGraphic. 2, 36, 37

EPSP Excitatory Post Synaptic Potential. 5

EVD Eigen Value Decomposition. 16

FDM Finite Difference Method. 22

FEM Finite Element Method. 22

fMRI functional Magnetic Resonance Imaging. 1

FOCUSS Focal under determined system solution. 25

HR-sLORETA Higher Resolution sLORETA. x, 45, 48-50, 52, 53

ICA Independent Component Analysis. 2, 10, 12-16, 28-30, 34, 45, 56

IPSP inhibitory post synaptic potential. 5 
JADE Joint Approximation Diagonalization of Eigenmatrices. 14, 32, 35, 56

LORETA Low Resolution Electromagnetic Tomography. 24

MCE Minimum Current Estimate. 25, 26

MEG Magnetoencephalography. vii, ix, 1, 2, 6-12, 15, 16, 18, 21, 24, 28-31, 33, 34, 36, 37, 56

MNDL Minimum Noiseless Description Length. 38, 40

MNE Minimum Norm Estimation. 23, 24, 26

MRI Magnetic Resonance Imaging. ix, 1, 21, 22

MSE Mean Square Error. viii, 42-44

MxNE Mixed-Norm Estimate. 25, 26

NoC Number of Component. viii, 34-36, 56

NoSEE Number of Source Eigenvalue Error. 33-35

OA Ocular Artifact. 28

PCA Principle Component Analysis. 12

PUS Percentage of Undetected Source number. viii, 42-44, 47, 48, 57

RMSE Root Mean Square Error. viii, 33-35

SD Spatial Dispersion. 47, 49, 52, 53, 57

sLORETA Standard Low Resolution Electromagnetic Tomography. x, 24, 41, 44-48, 55, 57

SNR Signal-to-Noise Ratio. x, 41-43, 46, 48, 49, 52, 54, 57

SQUID Superconducting Quantum Interference Device. 7

SSP Signal Space Projection. viii, 2, 10, 12, 15, 28-30, 32, 34-37, 56

STF Space-Time-Frequency. 26

STFT Short Time Fourier Transform. 26

STWV Space-Time-Wave-Vector. 26

SVD Singular Value Decomposition. 27 
VB Variational Bayesian. 26

WGN White Gaussian Noise. 30, 39, 55

WMNE Weighted Minimum Norm Estimate. 24, 26, 41, 54 